\title{
Thermal coefficients of methyl groups within ubiquitin and metabolic coupling of NAA and lactate in cortical neurons
}

\author{
Dissertation \\ for the award of the degree \\ "Doctor rerum naturalium" \\ of the Georg-August-Universität Göttingen
}

within the doctoral program molecular physiology of brain (CMPB)

of the Georg-August University School of Science (GAUSS)

submitted by

Davood Bakhtiari

from Kermanshah

Göttingen 2013 
Members of Thesis Committee and Examination Board:

First Reviewer:

Prof. Dr. Christian Griesinger

Department of NMR-based Structural Biology,

Max Planck Institute for Biophysical Chemistry, Göttingen, Germany

Second Reviewer:

Prof. Dr. Marina Bennati

Electron Spin Resonance Spectroscopy Group

Max Planck Institute for Biophysical Chemistry, Göttingen, Germany

Prof. Dr. Dirk Görlich

Department of Cellular Logistics,

Max Planck Institute for Biophysical Chemistry, Göttingen, Germany

Dr. Sebastian Kügler

Department of Neurology, Viral Vector Laboratory,

Göttingen University Medical School, Germany

\section{Members of Extended Thesis Committee and Examination Board:}

\section{Prof. Dr. Klaus-Armin Nave}

Department of Neurogenetics,

Max Planck Institute for Experimental Medicine, Göttingen, Germany

Prof. Dr. Kai Tittmann

Department of Bioanalytics,

George-August-Universität Göttingen, Germany

\section{Dr. Lars Kuhn}

NMR spectroscopy group,

European Neuroscience Institute, Göttingen, Germany

Date of the oral examination, $\mathbf{6}^{\text {th }}$ of September, 2013 


\section{Affidavit:}

Hereby I declare that my thesis entitled: “Thermal coefficients of methyl groups within ubiquitin and metabolic coupling of NAA and lactate in cortical neurons" has been written independently and with no other sources and aids other quoted.

Davood Bakhtiari, Göttingen, $14^{\text {th }}$ of July, 2013 


\section{Acknowledgement:}

First of all I would like to express the deepest appreciation to my supervisor, Prof. Dr. Christian Griesinger, who gave me the honor to work in his group with different elegant and challenging projects. His way of approaching the problems and teaching style made a magnificent impact on my scientific practice and understanding of biophysics and NMR.

I would like to thank Prof. Dr. Dirk Görlich and Dr. Sebastian Kügler who accepted to be member of my thesis committee and giving me helpful advices during this thesis. My special thanks go to Prof. Dr. Marina Bennati who accepted to be the second reviewer of this thesis. I also appreciate and thank my examination board Prof. Dr. Klaus-Armin Nave, Prof. Dr. Kai Tittmann and Dr. Lars Kuhn, despite their tight schedule agreed to evaluate my dissertation.

I am highly indebted to our collaborators Prof. Dr. Klaus-Armin Nave and Dr. Kathrin Kusch for the outstanding biological question, tedious sample preparation work and hours of fruitful discussion that pertain work that constitutes a large part of this thesis.

My special gratitude and thanks go to my colleague and mentor Dr. T. Micheal Sabo, without his significant contribution, patience and kind help with respect to the first project in this thesis would of made it impossible. Dr. Donghan Lee's idea was the crucial point for the first part of this thesis.

The colleagues of molecular biology and chemisty laboratory, Dr. Stefan Becker, Dr. Andrei Leonov and Karin Giller supported my thesis with the preparation of precious samples.

Special thanks to Dr. Nasrollah (Hessam) Rezaei-Ghaleh, who was always open and patient to my countless questions and never left any of them unanswered.

In addition I have to thank my best friends and colleagues, Drs. Luigi Russo, Adam Mazur, Holger Schmidt, Stefan Bibow for all their helps and discussions during my Ph.D. period.

I had a great time together with my friends and fellow Ph.D . students of the NMR-based structural biology department, David Ban, Jean-Phillip Demers, Hariandranath Kadavath, Hannes Fasshuber and Sheng-Qi Xiang. 


\section{Table of Contents}

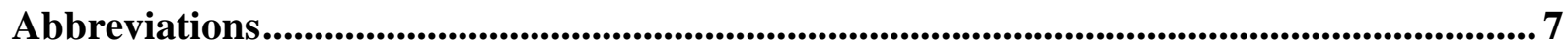

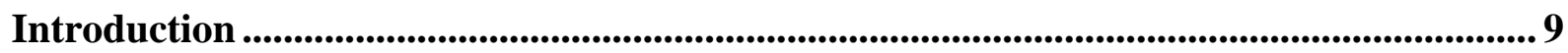

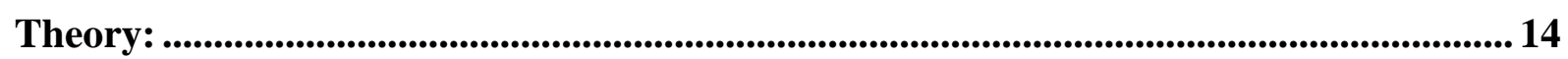

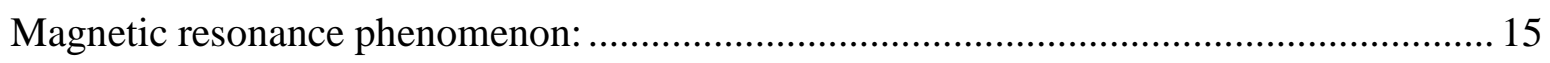

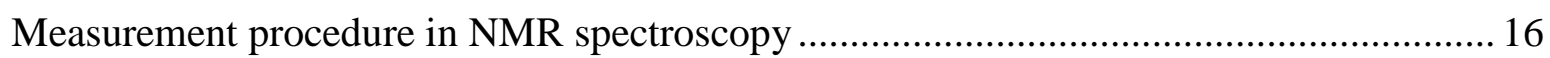

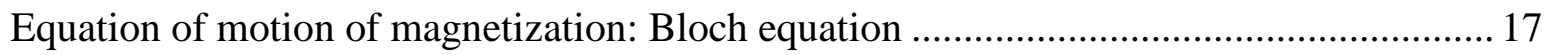

Precession of transverse magnetization and rotating frame reference system ................... 17

Continuous wave irradiation in rating frame to generate to transverse magnetization........ 19

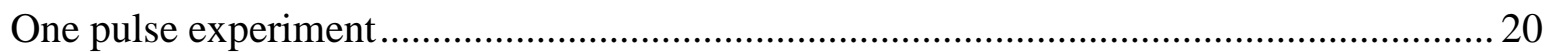

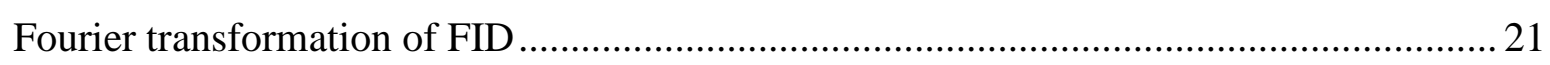

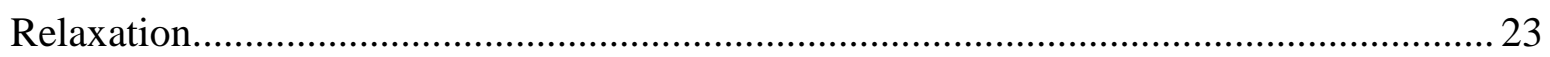

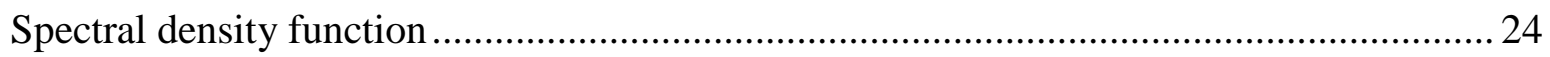

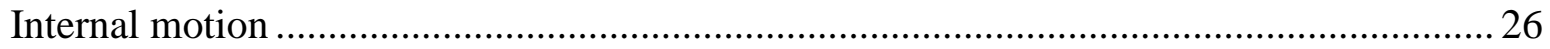

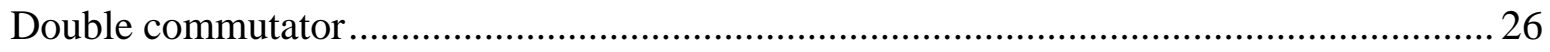

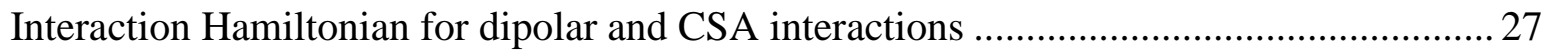

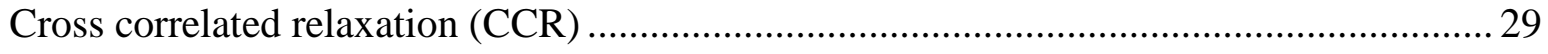

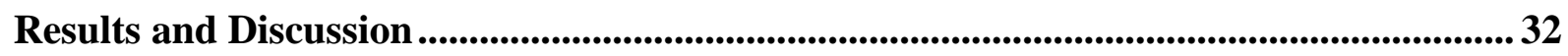

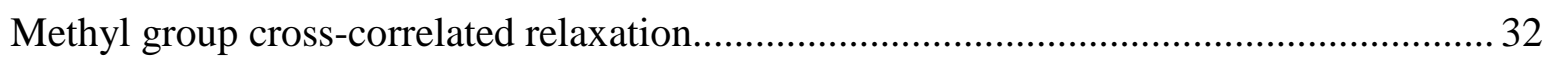

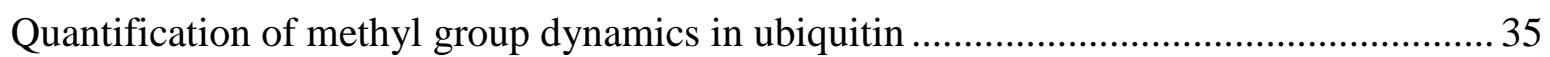

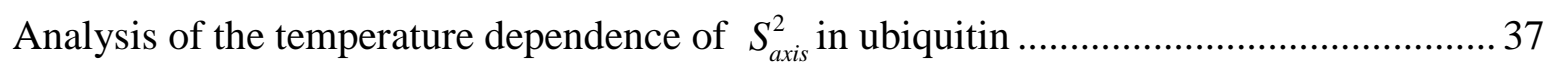

Insight into the thermal stability of ubiquitin from the distribution of thermal coefficients 44

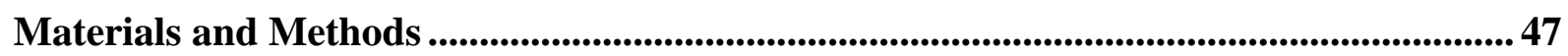

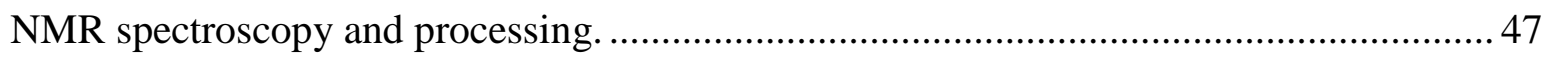

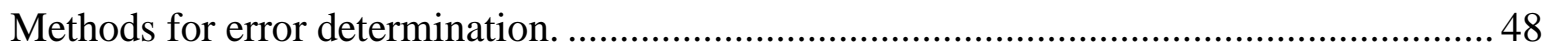

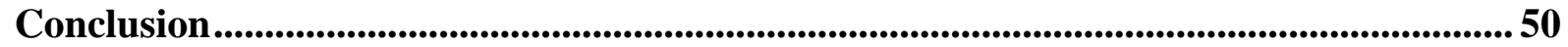

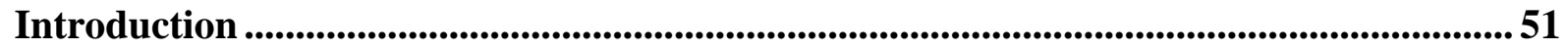

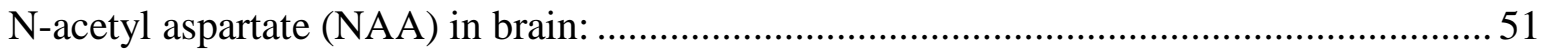

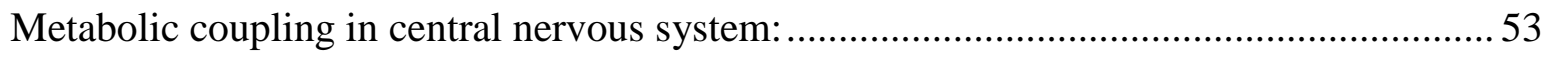

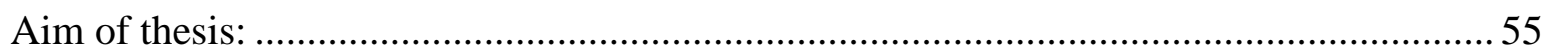

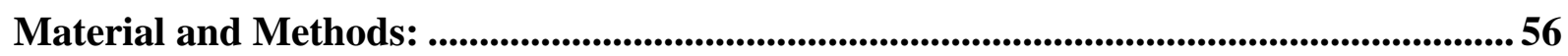

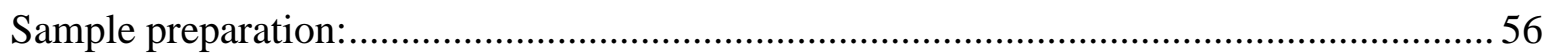

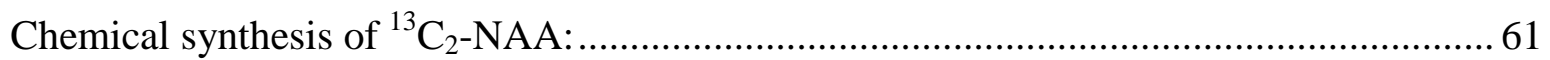

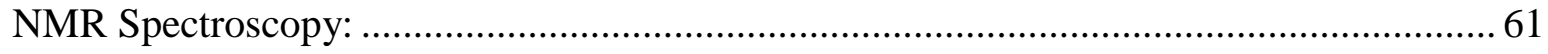

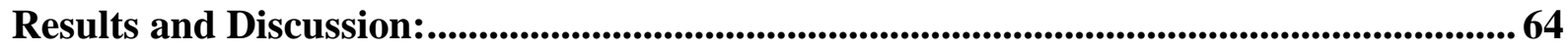


Quantification of NAA using NMR

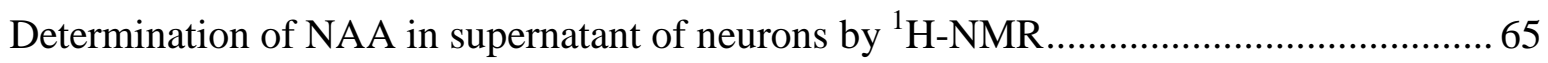

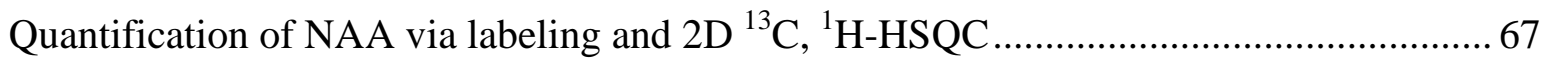

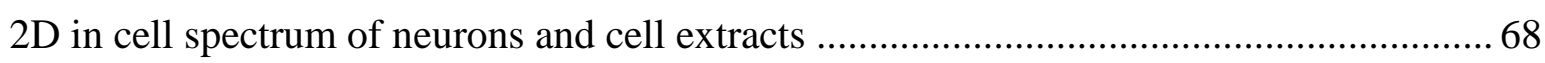

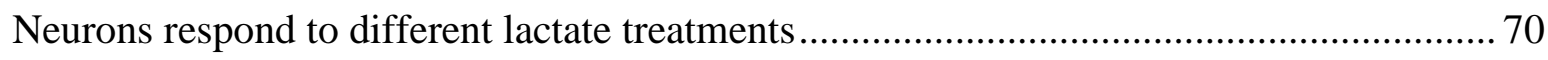

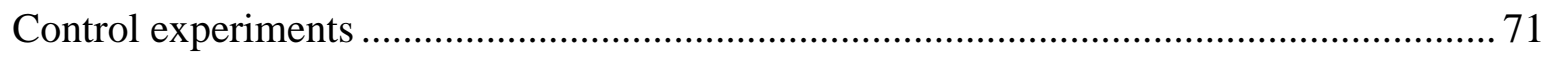

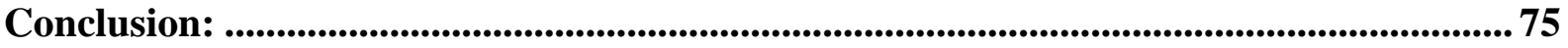

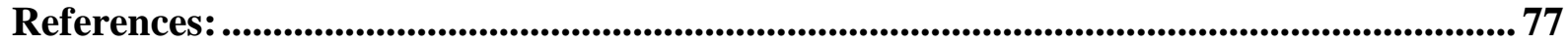

Appendix I...................................................................................................................................... 86

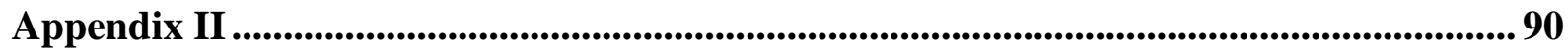

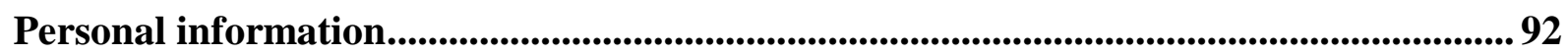

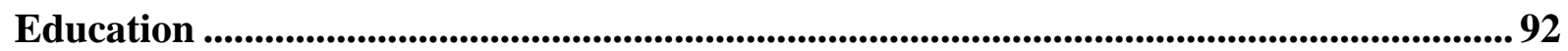

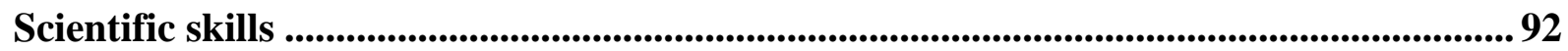

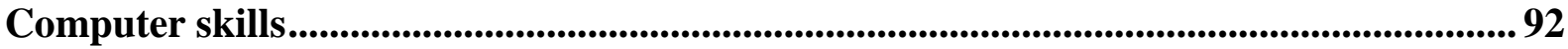

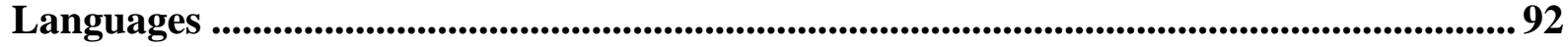

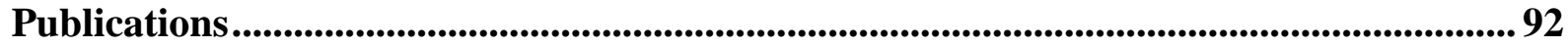




\section{Abbreviations}

1,3-BPG

2-PG

3-PG

Ac-CoA

ASPA

CCR

CNS

$C_{p}$

CSF

DAPI

DHAP

DMEM

DSS

F-1,6-BP

F6P

FCS

G6P

GAP

Glc
1,3-Biphosphoglycerate

2-Phosphoglycerate

3-Phosphoglycerate

Acetyl Coenzyme A

Aspartoacylase

Cross Correlated Relaxation

Central Nervous System

Heat capacity (constant pressure)

Cerebrospinal fluid

4',6-diamidino-2-phenylindole

Dihydroxy acetone phosphate

Dulbecco’s modified Eagle Medium

4,4-dimethyl-4-silapentane-1-sulfonate

Fructose 1,6-biphosphate

Fructose 6-phosphate

Fetal Calf Serum

Glucose 6-phosphate

Glyceraldehyde phosphate

Glucose 
Multiple sclerosis

NAA

N-Acetyl Aspartate

NAAG

N-Acetyl Aspartyl Glutamate

NAGA

N-Acetyl Glucoseamine

NAT-8L

$\mathrm{N}$-aspartoacetyl transferase 8L

NB

Neurobasal medium

NMR

Nuclear Magnetic Resonance

NOE

Nuclear Overhauser Effect

PEP

Phosphoenol pyruvate

$S_{\text {conf }}$

Conformational entropy

$\mathrm{T}_{1}$

Longitudinal relaxation time

$\mathrm{T}_{2}$

Transverse relaxation time

TCA

Tricarboxylic acid

TPPI

Time Proportional Phase Incrementation 


\section{Part I Thermal Coefficients of methyl groups within ubiquitin}

\section{Introduction}

The complex relationship between structure, function and dynamics strongly influences such fundamental physiological processes as protein folding, molecular recognition, and thermal stability. All of these procedures take place with simultaneous changes in the thermodynamic parameters of the system, specifically the enthalpy $(H)$ and the entropy $(S)$. Heat capacity $\left(C_{p}\right)$ represents the connection between the temperature dependence of $H$ and $S$ (Gomez et al.,1995; Prabhu and Sharp, 2005).

$$
\begin{gathered}
\Delta H=\int C_{p} d T \\
\Delta S=\int C_{p} d(\ln (T))
\end{gathered}
$$

The $C_{p}$ of a protein contributes in hydration of solvent exposed surface area, covalent bonds, electrostatic interactions, and hydrogen bonds. Furthermore, the local conformational entropy ( $S_{\text {conf }}$ ) of residues within a protein must also be considered. The term $S_{\text {conf }}$ is directly linked to the degrees of freedom each residue possesses within the three-dimensional protein structure. In order to have insights into the role of local dynamics in protein stability, interpretation of $C_{p}$ is needed. This covers the dependence of $S_{\text {conf }}$ on temperature. Statistical mechanics is widely used to connect the macroscopic properties of a system such as Helmholtz free energy, A, or the entropy, S, with the microstates (Nash, 1974),

$$
S=-\left(\frac{\partial A}{\partial T}\right)_{V}
$$

and

$$
A=-k_{B} T \ln Z_{T O T}
$$


Where $\mathrm{T}$ is the absolute temperature, $k_{B}$ is the Boltzmann's constant and $Z_{T O T}$ indicates the partition function of the system to evaluate all possible thermodynamic properties of the system (Yang and Kay, 1996). If all molecules of the system are decoupled from each other, $Z_{T O T}$ can be written in terms of molecular partition function, $\mathrm{Z}$, and the number of identical molecules, $\mathrm{N}$, as

$$
Z_{T O T}=\frac{Z^{N}}{N !}
$$

Where the $N$ ! factor is for correction of over counting the number of distinguishable states in the system (Nash, 1974). $Z$ can be decomposed into two partition functions describing contribution of bond vector fluctuations, $Z_{O}$ and the remaining contribution from all other sources, $Z_{U}$ (Yang and Kay, 1996)

$$
Z=Z_{U} Z_{O}
$$

And assuming each fluctuating bond vector is independent of all others,

$$
\begin{gathered}
Z=Z_{U} \prod_{j} z(j) \\
z(j)=\sum_{i} \exp \left\{-E_{i}(j) /\left(k_{B} T\right)\right\}
\end{gathered}
$$

Where $z(j)$ is the $j$ th bond vector partition function (with quantum mechanical approach) and the summation is over all $i$ energy energy states $\left(E_{i}\right)$ of the bond vector. It should be noted that the assumption of independence of bond vector motion might result in an over estimation on partition function calculation (Akke et al, 1993).

The total entropy of the system can be calculated by (Yang and Kay, 1996)

$$
\begin{gathered}
S_{\text {TOT }}=N\left(S_{U}+S_{O}\right)-k_{B} \ln N ! \\
S_{O}=\sum_{j} \frac{\partial\left\{k_{B} T \ln z(j)\right\}}{\partial T} \\
S_{O}(j)=\frac{\partial\left\{k_{B} T \ln z(j)\right\}}{\partial T}
\end{gathered}
$$


Where $S_{O}$ and $S_{U}$ are the entropy contribution to the total entropy per molecule from motions described above for the partition functions, $Z_{O}$ and $Z_{U}$. $S_{O}(j)$ indicates the component of $S_{O}$ originating from bond vector $j$. Using the well known Boltzmann equation and $z(j)$, the entropy for the $j$ th bond can be written as(Yang and Kay, 1996)

$$
S_{O}(j)=-k_{B} \sum_{i} p_{i}(j) \ln \left\{p_{i}(j)\right\}
$$

Where,

$$
p_{i}(j)=\frac{\exp \left\{-E_{i}(j) /\left(k_{B} T\right)\right\}}{\sum_{i} \exp \left\{-E_{i}(j) /\left(k_{B} T\right)\right\}}
$$

With the classical approach the energy difference between two successive states is assumed to be much less than the thermal energy $k_{B} T$ and therefore there is a continuum of states. Under this assumption the conformational entropy can be written as (Yang and Kay, 1996)

$$
S_{\text {conf }}(j)=-k_{B} \int_{V} p(q) \ln p(q) d V
$$

Where $d V=\sin \theta d \theta d \phi$ and the probability density function, $p(q)$, is

$$
p(q)=\frac{\exp \left\{-U(q) /\left(k_{B} T\right)\right\}}{\int_{V} \exp \left\{-U(q) /\left(k_{B} T\right)\right\} d V}
$$

Later in this work it will be shown, how the conformational entropy can be estimated experimentally by study of relaxation in nuclear magnetic resonance (NMR) spectroscopy.

NMR spectroscopy is a unique and powerful tool for characterizing such local dynamics with atomic resolution on multiple time-scales. Analysis of spin-lattice relaxation $\left(T_{1}\right)$, spin-spin relaxation $\left(T_{2}\right)$, and steady-state nuclear Overhauser enhancements (NOE) has been studied (Kay et al., 1989) by the “model free” formalism introduced by Lipari and Szabo (1981a and 1981b). This allows the extraction of a generalized order parameter $\left(S^{2}\right)^{1}$ that characterizes the amplitude of the individual bond vector motions on the ps to ns time-scale(

\footnotetext{
${ }^{1}$ It should be noticed that the notation "S" in the order parameter $S_{\text {axis }}^{2}$ and in the conformational enthropy $S_{\text {conf }}$ is not indicating the same concept and in order to follow the notations in the literature were kept the same.
} 
Lipari and Szabo, 1981a and 1981b). The generalized order parameters satisfy the inequalitiy, $0 \leq S^{2} \leq 1$, where lower values describe larger magnitude of internal motion.

Conformational entropy can be described by $S^{2}$ using the population distribution of bond vector orientations (Akke et al., 1993; Li et al., 1996; Yang and Kay, 1996) Study of temperature dependence of $S^{2}$ is possible by measurement of relaxation parameters at multiple temperature points. The local $C_{p}$ for each bond vector can be extracted from temperature dependence of $S^{2}$ (Yang et al., 1997; Mandel et al., 1996; Vugmeister et al., 2002; Vinther et al. 2011; Seewald et al. 2000).

Methyl groups are important probes for providing fundamental insight into protein folding, stability, and recognition. The hydrophobic core of the proteins typically accommodates many methyl groups, which are well dispersed throughout the core and show a wide variety of motional amplitudes (Yang et al., 1997; Lee and Wand, 2001; Lee et al., 1997; Sharp et al., 2002, Song et al., 2007). Hence, determination of the methyl group order parameters $\left(S_{a x i s}^{2}\right)$ disentangles the understanding how methyl dynamics on the ps to ns time scale are related to protein stability. Principally the $S_{a x i s}^{2}$ describes the motion for axis of symmetry ( $\mathrm{c}_{3}$-symmetry) within methyl group. This axis is along the bond connecting methyl group carbon to its previous atom and the mobility of the C-C bond is reflected in the amplitude of $S_{a x i s}^{2}$. Deuterium relaxation studies are typically employed for obtaining of $S_{a x i s}^{2}$ of methyl groups. For these measurement the methyl groups within the protein are mainly deuterated $\left(-\mathrm{CH}_{2} \mathrm{D}\right.$ or $\left.-\mathrm{CHD}_{2}\right)($ Muhandiram et al., 1995; Liao et al., 2012; Miller et al., 2002) Utilization of cross-correlated relaxation (CCR) rate between dipolar couplings of two $\mathrm{CH}$ bonds, $\sigma$,) in the methyl group for extracting $S_{a x i s}^{2}$ is an alternative method to ${ }^{2} \mathrm{H}$ relaxation approach(Liu et al., 2003; Zhang et al., 2006). 
Determination of $S_{a x i s}^{2}$ from $\sigma$ versus deuterium relaxation is essentially advantageous in the savings in measurement time, especially important for measuring $S_{a x i s}^{2}$ at many different temperatures. In previous studies, $S_{a x i s}^{2}$ calculated at one temperature point with deuterium relaxation studies could take up to one week. The time demand in such experiments is due to the requirement of data being acquired at two fields (Muhandiram et al., 1995). Recently a more rapid method was developed in determining $S_{a x i s}^{2}$ from the measurement of 5 relaxation rates for $-\mathrm{CH}_{2} \mathrm{D}$ (Millet et al., 2002) and 4 relaxation rates for $-\mathrm{CHD}_{2}$ (Liao et al., 2012) to extract the same information to approximately one day. Here, $S_{a x i s}^{2}$ derived from $\sigma$ can require as little as one hour of measurement time. This method is therefore perfectly suited for studying the temperature dependence of $S_{a x i s}^{2}$.

The temperature dependence of the methyl group order parameters is reported here within ubiquitin. The order parameters were derived from the cross-correlated relaxation between dipolar couplings of two $\mathrm{CH}$ bonds. Thermal coefficients are calculated from these experiments and characterizing this temperature dependence, specifically the characteristic thermal coefficient $\Lambda$ and the local heat capacity $C_{p}$. Furthermore, we analyze and compare the distribution of methyl group thermal coefficients within ubiquitin, illustrating the relatively high thermostability of the N-terminal region of this protein. 


\section{Theory:}

NMR spectroscopy is a powerful tool for a variety of applications in chemistry, physics, biology and many other disciplines. The area of application of NMR spectroscopy is divided into three parts:

1. NMR-spectroscopy of liquids and solutions (high resolution NMR) in chemistry, structural biology and metabolomics

2. Solid state NMR spectroscopy in chemistry, structural biology and material sciences

3. NMR spectroscopy and imaging of more or less intact living species (MRI: Magnetic Resonance Imaging and MRS: Magnetic Resonance Spectroscopy) in medicine, physiology and biology

The first approach is the main branch for study of the structure and dynamics of molecules in atomic resolution. The second direction characterizes the molecules in solid state, which in contrast with X-Ray crystallography need not be in crystalline form. The third area, especially tomography, has a huge impact in medicine for investigation of soft tissue (brain, spinal cord as widely used in diagnostics and other organs). In this thesis the high resolution NMR spectroscopy was utilized to study the dynamics of proteins (Part I) and to study metabolomics within cortical neurons (Part II).

Depending on which parameter (e.g. chemical shift, scalar coupling and relaxation etc.) being investigated, different kind of information (e.g. constitution, stereochemistry and dynamics etc.) can be extracted about the molecule of interest. 


\section{Magnetic resonance phenomenon:}

There are almost 100 nuclei isotopes which possess magnetic moment and called nuclear spin. For the Spin $\overrightarrow{\boldsymbol{I}}$ there is a proportional magnetic momentum $\vec{\mu}$, and the gyromagnetic ratio, $\gamma$, is the proportionality constant $(\vec{\mu}=\gamma \hbar \vec{I})$. The magnetic moment can orient itself along a magnetic field like a bar magnet since the parallel orientation of $\overrightarrow{\boldsymbol{B}}$ and $\overrightarrow{\boldsymbol{\mu}}$ is energetically favored. The nuclear spins are allowed to accept just limited number of orientations, namely $2 I+1$ states. For example a spin with $I=1 / 2$ there are $2 * 1 / 2+1=2$ states available. If the energy of one spin is observed in an external magnetic field along $z$ axis, i.e. $\overrightarrow{\boldsymbol{B}}=B_{0} \overrightarrow{\boldsymbol{e}}_{\mathbf{z}}$, then we have

$$
E=-\vec{\mu} \vec{B}=-\gamma \hbar \vec{I} \vec{B}=-\gamma \hbar I_{z} B_{0}
$$

where $B$, the external magnetic field, is conventionally set parallel to the z-axis. For spin $\frac{1}{2}$ the energy favored state is called $\alpha$ state with $\left\langle I_{z}\right\rangle=m_{z}=\frac{1}{2}$ and the higher energy level with $\left\langle I_{z}\right\rangle=m_{z}=-\frac{1}{2}$ called $\beta$ state (Fig. I). The NMR spectroscopy utilizes the energy difference between these two energy levels to generate spectral transitions. The frequency of the transition is given by

$$
\Delta E=\hbar \omega=\hbar \gamma B_{0} \text { or } v=\frac{\gamma B_{0}}{2 \pi}
$$

This is the fundamental equation for resonance in the NMR spectroscopy. The NMR spectrum consists of the frequencies of transitions and their intensities.

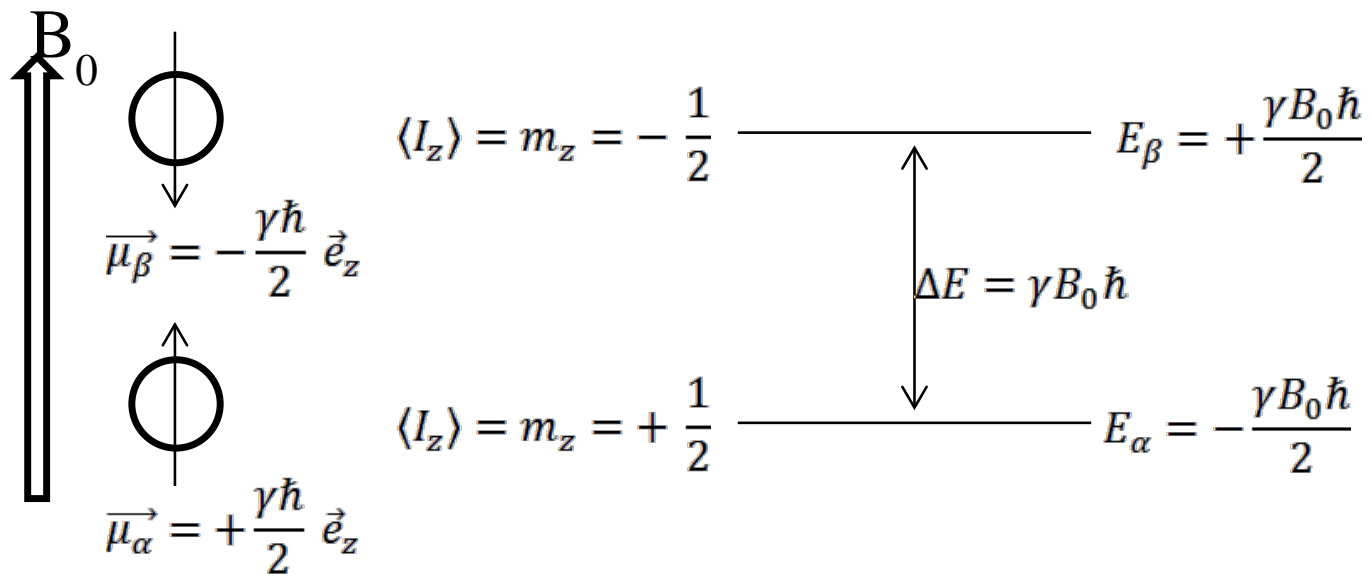


For the proton $1 \mathrm{H}$ the energy difference in a magnetic field strength of $14.09 \mathrm{~T}$ and gyromagnetic ratio of $2.6751 \times 10^{8} \mathrm{~Hz} \mathrm{~T}^{-1}$ is given by $\Delta E=N_{A} \gamma B_{0} \hbar=240 \mathrm{~mJ} / \mathrm{mol}$. This energy is in comparison with the thermal energy at the room temperature $R T=2.3 \mathrm{~kJ} / \mathrm{mol}$, four orders of magnitude smaller. Considering Boltzmann distribution, it can be calculated that at room temperature just one molecule out of 20000 is being observed. This shows that NMR is principally a rather non-sensitive technique. The sensitivity, however, can be increased by measurement at higher external fields, using nuclei with high gyromagnetic ratio, preparing probes with possible high concentration and lowering the noise level by employing so called cryogenic probe heads.

\section{Measurement procedure in NMR spectroscopy}

According to the fundamental resonance condition in NMR spectroscopy: $v=\frac{\gamma B_{0}}{2 \pi}$, an irradiation of a fluctuating magnetic field, like a radio frequency, with the corresponding frequency is absorbed by spins to equate the population of both energy states, which corresponds to rotation of the magnetization from z-axis to the transverse plane. The transverse magnetization, $\mathrm{M}^{+}(\mathrm{t})$, in presence of the static magnetic field experiences a torque, which is equal to the time derivative of the angular momentum (Cavangagh et al., 2007). This torque leads the transverse magnetization, $\mathrm{M}^{+}(\mathrm{t})$, to ratate about the $\mathrm{z}$-axis and observability of the signal. 


\section{Equation of motion of magnetization: Bloch equation}

The interaction of the external magnetic fields, $\vec{B}$, on the generated magnetization from spins in $B_{0}$ has been studied by Felix Bloch, who developed equation of motion for magnetization that is called Bloch equation after him:

$$
\overrightarrow{\dot{M}}=\frac{d \vec{M}}{d t}=\frac{d}{d t}\left(\begin{array}{l}
M_{x} \\
M_{y} \\
M_{z}
\end{array}\right)=\gamma(\vec{M} \times \vec{B})-\Gamma\left(\vec{M}-M_{0} \vec{e}_{z}\right)
$$

The equation of motion for magnetization is an Euler circular motion equation, which is extended with a relaxation term $\Gamma$. The relaxation tern describes the return of magnetization to the Boltzmann equilibrium $\left(M_{0}\right)$,

$$
\Gamma=\left(\begin{array}{ccc}
1 / T_{2} & 0 & 0 \\
0 & 1 / T_{2} & 0 \\
0 & 0 & 1 / T_{1}
\end{array}\right)
$$

where $T_{1}$ and $T_{2}$ are longitudinal and transversal relaxation times respectively.

\section{Precession of transverse magnetization and rotating frame reference system}

For a Boltzmann magnetization in the external field along $\mathrm{z}$ axis, if we ignore the relaxation at this point, we have

$$
\overrightarrow{\dot{M}}=\gamma M_{0} \vec{e}_{z} \times B_{0} \vec{e}_{z}=0
$$

which means that the Boltzmann magnetization is time invariant and stays conserved. In case of the transverse magnetization containing $M_{x}$ and $M_{y}$, however, there are changes with the time. Without relaxation we can write for the transverse magnetization $\left(\vec{B}=B_{0} \vec{e}_{z} ; \gamma M_{0}=\right.$ $\omega_{L}=2 \pi v_{L} ; \omega_{L}$ is Larmor circular frequency) in a component wise notation with $\vec{M}=$ $M_{x} \vec{e}_{x}+M_{y} \vec{e}_{y}+M_{z} \vec{e}_{z}$

$$
\frac{d M_{x}}{d t}=\gamma\left[\vec{M} \times B_{0} \vec{e}_{z}\right]_{x}=\gamma B_{0} M_{y}
$$




$$
\frac{d M_{y}}{d t}=\gamma\left[\vec{M} \times B_{0} \vec{e}_{z}\right]_{y}=-\gamma B_{0} M_{x}
$$

By differentiation of both equations and substitution we obtain

$$
\begin{aligned}
& \frac{d^{2} M_{x}}{d t^{2}}=-\left(\gamma B_{0}\right)^{2} M_{x} \\
& \frac{d^{2} M_{y}}{d t^{2}}=-\left(\gamma B_{0}\right)^{2} M_{y}
\end{aligned}
$$

These equations are the classical oscillation equation and their solution is (with $\omega_{L}=\gamma B_{0}$ ),

$$
\begin{aligned}
& M_{x}(t)=M_{x}(0) \cos \left(\omega_{L} t\right)-M_{y}(0) \sin \left(\omega_{L} t\right) \\
& M_{y}(t)=M_{x}(0) \sin \left(\omega_{L} t\right)+M_{y}(0) \cos \left(\omega_{L} t\right)
\end{aligned}
$$

Both equations can be written in a matrix notation,

$$
\left(\begin{array}{l}
M_{x}(t) \\
M_{y}(t)
\end{array}\right)=\left(\begin{array}{cc}
\cos \left(\omega_{L} t\right) & -\sin \left(\omega_{L} t\right) \\
\sin \left(\omega_{L} t\right) & \cos \left(\omega_{L} t\right)
\end{array}\right)\left(\begin{array}{l}
M_{x}(0) \\
M_{y}(0)
\end{array}\right)
$$

The matrix which multiplied by the vector $\left(\begin{array}{l}M_{x}(0) \\ M_{y}(0)\end{array}\right)$, is called rotation matrix with the rotation angle of $\omega_{L} t$. The magnetization rotates about the $B_{0}$ field with $\omega_{L}=\gamma B_{0}$. This precession with the Larmor frequency is valid for all types of nuclei in the same manner. Due to the different chemical environment (electronic shielding) about the nuclei, they feel the external magnetic field slightly different $B_{0}\left(1-\sigma_{i s o}^{i}\right)$ and their corresponding circular frequency deviates from Larmor frequency, i.e. $\omega_{i}=\gamma B_{0}\left(1-\sigma_{i s o}^{i}\right)$, where $\sigma_{i s o}^{i}$ is the isotropic chemical shift of nucleus $i$ in ppm. In order to separate these small deviations from the Larmor frequency in $\mathrm{MHz}$ order and to observer the small effects of chemical shift in clearly, a rotating coordination system is introduced, which rotates with the constant circular frequency of $\omega_{0}$. The rotation frequency should be slightly different from precession frequency of observed nuclei $\omega_{i}$ (i.e. $\omega_{0}$ is in $\mathrm{MHz}$ and $\omega_{i}-\omega_{0}=\Omega \mathrm{in} \mathrm{kHz}$ ). The frequency of rotating coordination system (rotating frame) is mainly not identical to the Larmor 
frequency $\omega_{L}=\gamma B_{0}$ ), since the chemical shifts are distributed unsymmetrical about the $\omega_{L}$ and the $\omega_{0}$ should be in the centre of chemical shifts span.

The Bloch equation can be rewritten in the rotating frame (Cavanagh et al., 2007),

$$
\frac{d \vec{M}}{d t}=\gamma\left(B_{0} \vec{e}_{z}(1-\sigma)-\frac{\omega_{0}}{\gamma} \vec{e}_{z}\right) \times \vec{M}-\Gamma\left(\vec{M}-M_{0} \vec{e}_{z}\right)
$$

And again in absence of relaxation we can write the solution for the Bloch equation in the rotating frame of reference with analogy to the static laboratory frame as

$$
\left(\begin{array}{l}
M_{x}(t) \\
M_{y}(t)
\end{array}\right)=\left(\begin{array}{cc}
\cos \left(\Omega_{i} t\right) & -\sin \left(\Omega_{i} t\right) \\
\sin \left(\Omega_{i} t\right) & \cos \left(\Omega_{i} t\right)
\end{array}\right)\left(\begin{array}{l}
M_{x}(0) \\
M_{y}(0)
\end{array}\right)
$$

\section{Continuous wave irradiation in rating frame to generate to transverse}

\section{magnetization}

The precession of transverse magnetization was discussed in the previous section and now the generation of transverse magnetization using a radio frequency (r.f.) irradiation will be described. Here we observe the irradiation of a radiofrequency with the carrier frequency of $\omega_{0}$ and field strength of $2 B_{1}$ during a finite time interval and in the transverse plane. In the laboratory frame this corresponds to an additional field $\left(2 B_{1} \vec{e}_{x} \cos \omega_{0}\right.$ t, where irradiation is applied along the $x$ axis). This field can be separated into two components

$$
2 B_{1} \vec{e}_{x}^{L} \cos \omega_{0} \mathrm{t}=B_{1}\left(\vec{e}_{x}^{L} \cos \omega_{0} \mathrm{t}+\vec{e}_{y}^{L} \sin \omega_{0} \mathrm{t}\right)+B_{1}\left(\vec{e}_{x}^{L} \cos \left(-\omega_{0} \mathrm{t}\right)+\vec{e}_{y}^{L} \sin \left(-\omega_{0} \mathrm{t}\right)\right)
$$

The first and second components represent two vectors, which rotate with the $\omega_{0}$ and $-\omega_{0}$ about the $z$ axis respectively. In the rotating frame the component that rotates with $\omega_{0}$ represents a fixed component with the field strength of $B_{1} \vec{e}_{x}$. The second component rotates with $-2 \omega_{0}$ in the rotating frame and the total $B_{1}$ field can be described as

$$
B_{1} \vec{e}_{x}+B_{1}\left(\vec{e}_{x} \cos \left(-2 \omega_{0} \mathrm{t}\right)+\vec{e}_{y} \sin \left(-2 \omega_{0} \mathrm{t}\right)\right)
$$


The counter rotating component changes its sign at e.g. $\frac{\omega_{0}}{2 \pi}=500 \mathrm{MHz}$ each $0.5 \mathrm{~ns}$ and has no significant effective on the magnetization. Therefore the remaining component is the static component $B_{1} \vec{e}_{x}$. The effective Bloch equation in the rotating frame is

$$
\begin{gathered}
\frac{d \vec{M}}{d t}=\gamma\left(B_{0} \vec{e}_{z}(1-\sigma)-\frac{\omega_{0}}{\gamma} \vec{e}_{z}+B_{1} \vec{e}_{x}\right) \times \vec{M}-\Gamma\left(\vec{M}-M_{0} \vec{e}_{z}\right) \\
=\left(\Omega \vec{e}_{z}+\gamma B_{1} \vec{e}_{x}\right) \times \vec{M}-\Gamma\left(\vec{M}-M_{0} \vec{e}_{z}\right) .
\end{gathered}
$$

In the on-resonance case $\left(\omega_{0}=\omega_{i} ; \Omega_{i}=0\right)$ within a rotating coordinate system the $z$ components of the field vanishes. By neglecting relaxation we have

$$
\dot{\vec{M}}=\gamma\left(B_{1} \vec{e}_{x} \times \vec{M}\right)
$$

The magnetization rotates about the axis of the radiofrequency field. If the applied field is along the $\mathrm{x}$ axis, the $\mathrm{z}$ magnetization starts to rotate about the $\mathrm{x}$ axis with $\omega_{1}=\gamma B_{1}$,

$$
\begin{aligned}
& M_{y}(t)=M_{y}(0) \cos \left(\omega_{1} t\right)-M_{z}(0) \sin \left(\omega_{1} t\right) \\
& M_{z}(t)=M_{y}(0) \sin \left(\omega_{1} t\right)+M_{z}(0) \cos \left(\omega_{1} t\right)
\end{aligned}
$$

\section{One pulse experiment}

A $90^{\circ}$ pulse is applied on a magnetization (e.g. Boltzmann magnetization $M_{z}(0)=$ $M_{0} ; M_{x, y}(0)=0$ ), if the duration of applied field, $\tau$, is set, so that the $\omega_{1} \tau=\frac{\pi}{2}$. After the pulse we have

$$
\begin{gathered}
M_{y}(\tau)=-M_{z}(0) \sin \left(\frac{\pi}{2}\right)=-M_{0} \\
M_{z}(\tau)=M_{y}(0) \sin \left(\frac{\pi}{2}\right)=0 .
\end{gathered}
$$

From now on in the real case of experiment the influence of relaxation cannot be ignored anymore. It is important that $\omega_{1}$ is much larger than $1 / T_{1}$ and $1 / T_{2}$ in order to have no influence from relaxation during the pulse and the $M_{0}$ is fully transferred onto the transverse plane. After the applied field is turned off, this transverse magnetization generated by $90^{\circ}$ 
pulse starts to rotate about the $B_{0}$ axis which is along $\mathrm{z}$ axis. Since we detect the magnetizations in the transverse plane, an oscillating signal is being observed which decays due to relaxation and generates the so called free induction decay or FID (Cavanagh et al., 2007).

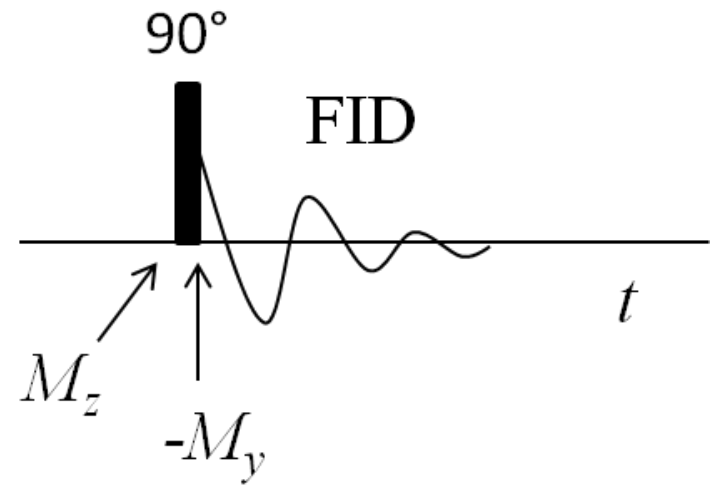

\section{Fourier transformation of FID}

After a $90^{\circ}$ pulse along $y$ axis at the time point $\mathrm{t}=0$ the original $z$-magnetization is on along the $x$ axis: $\vec{M}(0)=M_{0} \vec{e}_{x}$. Different magnetizations generate the superposition of different damped harmonic oscillators. Here the FID of only one spin is described. $M_{x}$ and $M_{y}$ are detected and complex added to $M^{+}=M_{x}+i M_{y}$. The x-component and y-component of the magnetization are taken as real and imaginary parts of the complex function,

$$
\begin{gathered}
M_{x}=M_{0} \cos \Omega_{i} t \\
M_{y}=M_{0} \sin \Omega_{i} t \\
M^{+}=M_{0}\left(\cos \Omega_{i} t+i \sin \Omega_{i} t\right)=M_{0} e^{i \Omega_{\mathrm{i}} \mathrm{t}}
\end{gathered}
$$

Now an exponential decay function originating from relaxation is multiplied with the $1 / T_{2}$ (the transverse relaxation rate constant to generate the time domain signal:

$$
f(t)=M_{0} e^{i \Omega_{\mathrm{i}} \mathrm{t}} \cdot e^{-\mathrm{t} / T_{2}}
$$

Fourier transformation is applied to generate the frequency domain signal, $F(\omega)$, 


$$
\begin{aligned}
F(\omega) & =\int_{0}^{+\infty} e^{-i \omega t} \cdot M_{0} e^{i \Omega_{\mathrm{i}} \mathrm{t}} \cdot e^{-\mathrm{t} / T_{2}} d t \\
= & \left.M_{0} \frac{e^{-i(\omega-\Omega) t-t / T_{2}}}{-i(\omega-\Omega)-1 / T_{2}}\right|_{0} ^{+\infty} \\
& =M_{0} \frac{1}{i(\omega-\Omega)+1 / T_{2}} \\
& =L(\omega)=A(\omega)+i D(\omega)
\end{aligned}
$$

The real part $A(\omega)$ and the imaginary part $D(\omega)$ of the Lorentz line are depicted below.

$$
\begin{aligned}
& A(\omega)=M_{0} \frac{1 / T_{2}}{\left(\omega-\Omega_{\mathrm{i}}\right)^{2}+\left(\frac{1}{T_{2}}\right)^{2}} \\
& D(\omega)=-M_{0} \frac{\omega-\Omega_{\mathrm{i}}}{\left(\omega-\Omega_{\mathrm{i}}\right)^{2}+\left(\frac{1}{T_{2}}\right)^{2}}
\end{aligned}
$$
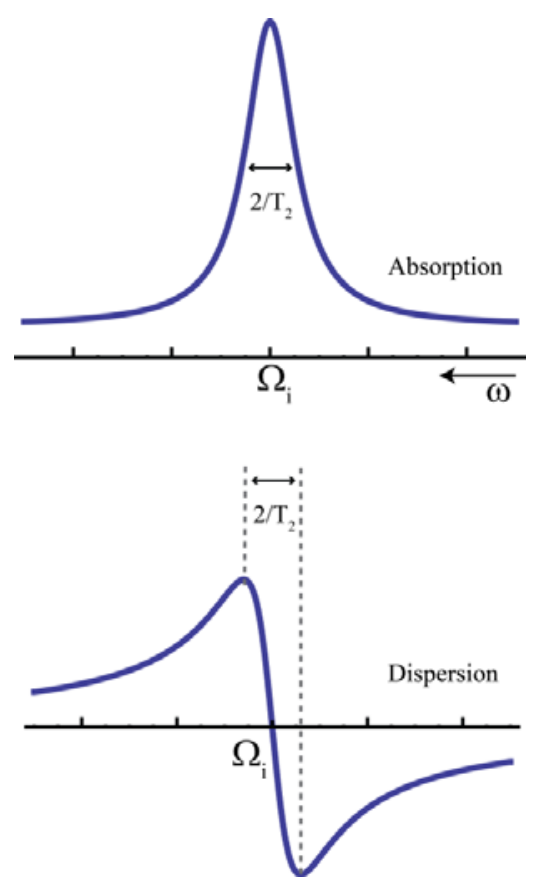

The absorption part has a maximum at the resonance frequency, i.e. $\Omega_{\mathrm{i}}$. The intensity is scaled by the initial magnetization $M_{0}$ as well as $T_{2}$. The line width at the half maximum is $2 / T_{2}\left(1 / \pi T_{2}\right.$ in frequency). In NMR spectroscopy absorption lines are mainly used as they decay slower than dispersion lines by distancing from resonance frequency. This leads to less overlapping when more signals are being studied. 
Here, in order to complete this section, the time variation of $z$ magnetization is described.

Immediately after the $90^{\circ}$ pulse there is no longitudinal magnetization: $M_{z}(t=0)=0$.

Substituting in the Bloch equation for the z-component in the rotating frame,

$$
\frac{d M_{z}}{d t}=-\Gamma_{\mathrm{z}}\left(M_{z}-M_{0}\right)
$$

This differential equation can be solved easily:

$$
M_{z}(t)=M_{0}\left(1-e^{-\Gamma_{\mathrm{z}} \mathrm{t}}\right)
$$

It means that the $z$ magnetization returns to the Boltzmann equilibrium with a rate constant $\Gamma_{z}$

That is valid for the definition of the longitudinal $T_{1}$ time: $\Gamma_{\mathrm{z}}=1 / \mathrm{T}_{1}$.

\section{Relaxation}

The fluctuating magnetic interactions of the observed spin cause the relaxation and can have various origins, e.g.

1. Paramagnetic particles in the sample $\left(\mathrm{O}_{2}\right.$, salts etc. $)$

2. Intermolecular dipolar coupling (the most important mechanism of relaxation)

3. Chemical shift anisotropy (CSA), which is more pronounced in higher magnetic fields $\left(B_{0}\right)$ and in specific nuclei $\left({ }^{19} \mathrm{~F},{ }^{31} \mathrm{P}\right)$

4. Quadrupolar coupling (particles with spin $I=1$ or coupled to spins with $I \geq 1$ )

5. Scalar coupling

We assume that in a two level scheme there is a fluctuating magnetic interaction which affects both energy levels. According to the Heisenberg uncertainty principle ( $\Delta E \times \Delta t \leq \hbar)$ the

lifetime of a transition, $\Delta t \leq \frac{\hbar}{\Delta E}=\frac{1}{\Delta \omega}$.It means that the lifetime is limited by the magnitude of the fluctuation of magnetic interaction (Abragam, 1961).

To understand the relaxation phenomenon two central concepts, 1) spectral density function and 2) double commutators, should be discussed here 


\section{Spectral density function}

Transitions between the spin levels after a perturbation like an excitation pulse return to the thermodynamically favored distribution. As described above this mechanism is characterized by the longitudinal relaxation time, $T_{1}$. The second process deals with the transverse magnetization or coherences, which are absent in the Boltzmann equilibrium. The decay of transverse magnetization is determined by the transversal relaxation time, $T_{2}$ A transition between the energy levels can be succeeded if there is a matched frequency with the energy difference from an external time variant perturbation. Therefore a so called spectral power density or spectral density function of the transition frequency is important for its relaxation. It shows how much electromagnetic power for a specific frequency is available. Relaxation can occur not only via detectable single quantum transitions, but also through non detectable double or zero quantum transitions. For calculation of the spectral density function we need first to define the correlation function, $G(t)$, of time variant magnetic interactions $h(t$ (Abragam, 1961):

$$
G(t)=\int_{-\infty}^{+\infty} h_{1}\left(t^{\prime}\right) \cdot h_{2}\left(t^{\prime}+t\right) d t^{\prime}
$$

Where the correlation function indicates the amplitude of the magnetic interaction $h_{1}\left(t^{\prime}\right)$ with itself (auto correlation; $h_{1}\left(t^{\prime}\right)=h_{2}\left(t^{\prime}\right)$ ) or with another magnetic interaction $h_{2}\left(t+t^{\prime}\right)$ which implicates the cross correlated relaxation. Since most the magnetic interactions such as the chemical shift anisotropy and dipolar coupling are determined by the orientation of molecule in the magnetic field, the correlation time which measures the decorrelation of the values of those magnetic interactions is important for relaxation and is defined by the reorientation time constant of the molecule $\left(\tau_{c}\right)$. The correlation function for all cases which being described here has the form (Abragam, 1961; Cavanagh et al., 2007)

$$
G(\tau)=\sum_{m=-2}^{2} \overline{Y_{2}^{m}[\Omega(\tau)] Y_{2}^{m}[\Omega(t+\tau)]}
$$


Where $Y_{2}^{0}$ is the modified second-order spherical harmonics (see table 1 ) and $\Omega(t)=$ $\{\theta(t), \phi(t)\}$ are the polar angels. The correlation function for isotropic rotational diffusion which we will use in all cases of the thesis has the form (Abragam, 1961; Cavanagh et al., 2007)

$$
G(t)=\frac{1}{5} e^{-|t| / \tau_{c}}
$$

where $\tau_{c}$ is the correlation time for the decaying function $G(t)$. The Fourier transformation of the correlation function, $G(t)$ is the spectral density function, $J(\omega)$, which has a Lorentzian line shape (Abragam, 1961),

$$
J(\omega)=\frac{2 \tau_{c}}{5\left(1+\omega^{2} \tau_{c}^{2}\right)}
$$

For the value of spectral density function it is important to know, with which frequency the transitions can be generated (see table 1) in the spin system, e.g. (in system of I and S)

- 0 or $\omega_{I}-\omega_{S}$ (zero quantum)

- $\omega_{I}$ or $\omega_{S}$ (single quantum)

- $2 \omega_{I}, 2 \omega_{S}$ or $\omega_{I}+\omega_{S}$ (double quantum)

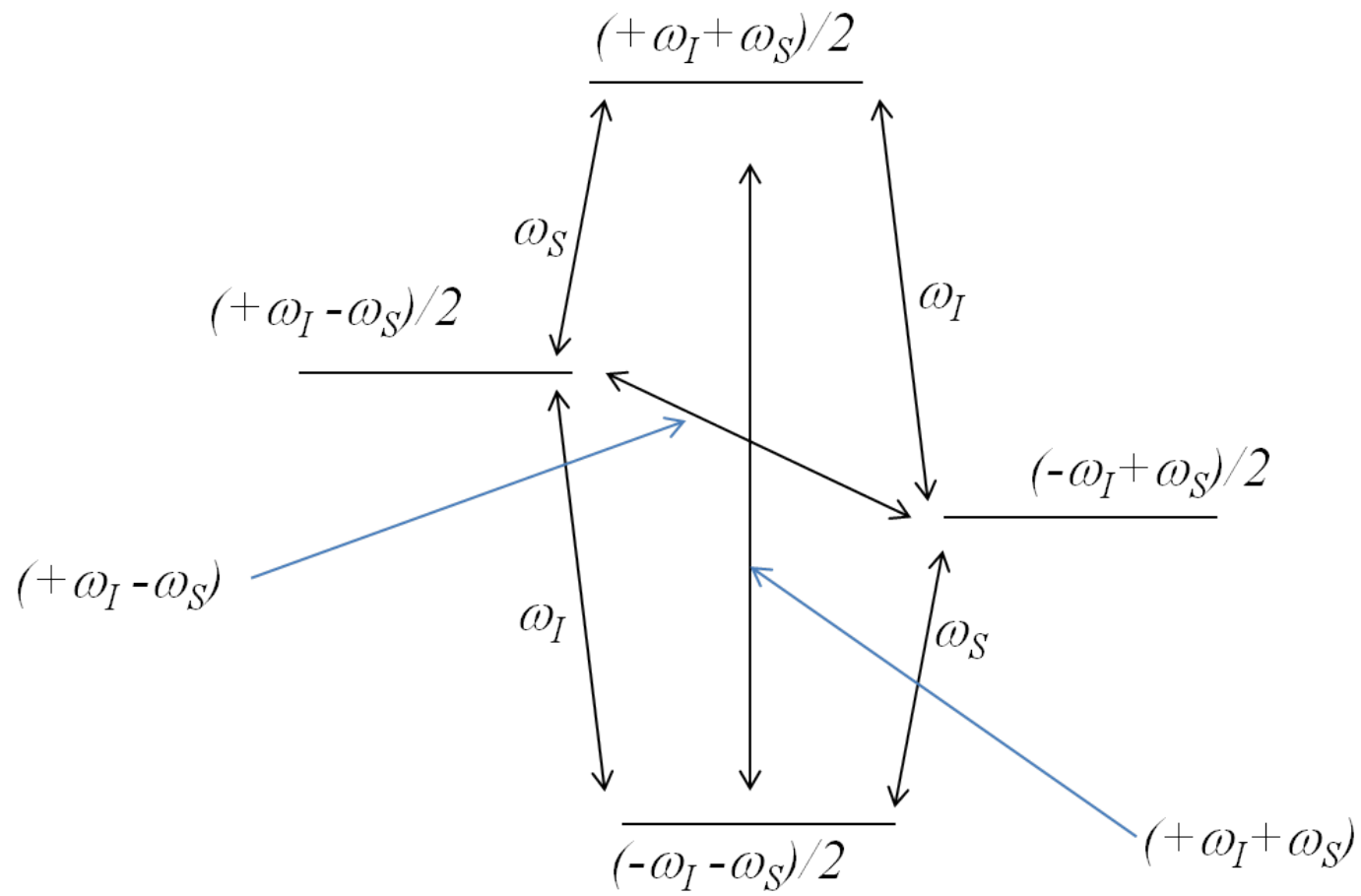




\section{Internal motion}

There are several models to describe the internal motion of molecules. The most common model developed by Lipari and Szabo (1981a and 1981b) assumes that each C-H or N-H vector has a $\tau_{c}$ independent motion. This kind of motion assumed to be very fast and the spectral density function can be changed into

$$
\begin{gathered}
J(\omega)=S^{2} J\left(\omega, \tau_{c}\right)+\left(1-S^{2}\right) J\left(\omega, \tau_{e}^{\prime}\right) \\
\frac{1}{\tau_{e}^{\prime}}=\frac{1}{\tau_{e}}+\frac{1}{\tau_{c}}
\end{gathered}
$$

where $S^{2}$ is the so called order parameter, $\tau_{c}$ is the global correlation time and $\tau_{e}$ is the correlation time for the fast motion. The order parameter, $S^{2}$, takes the maximum value, 1 , in absence of local motion and is equal zero for freely mobile vector. We can also suppose that $\tau_{e}^{\prime}$ is very small compared to $\tau_{c}$, therefore the $\omega$-dependence of $J\left(\omega, \tau_{e}^{\prime}\right)$ vanishes and we have $\frac{1}{\tau_{e}^{\prime}}=\frac{1}{\tau_{e}}$. To estimate the value of order parameter it is essential to experimentally measure different relaxation parameters (e.g. $T_{1}, T_{2}$, NOE and cross correlated relaxation etc.).

\section{Double commutator}

The spectral density function gives the frequency of the transition in the spin system, which is excited by the fluctuating magnetic interactions. Determination of transitions that are being relaxed by fluctuating magnetic interaction can be described by perturbation theory. This depends on the transition moment, which is generated by the perturbing operator between the corresponding spin states. Using Liouville von Neumann equation, $(\dot{\rho}=-i \hbar[\widehat{H}, \rho] ; \rho$ is density matrix and $\widehat{H}$ is interaction Hamiltonian), the time evolution of density matrix can be described. Here the time evolution of the density matrix and the transitions made by relaxation arises from double commutation of the perturbing Hamiltonian operator. The 
excitable transitions can be determined by substitution in the corresponding double commutator. Thereby we obtain from the initial state $\rho$ the final state $\rho$ ' by creating of the double commutator from $\rho$ time dependent perturbing Hamiltonian $\widehat{H}_{\text {int }}(t)$ and $\widehat{H}_{\text {int }}\left(t^{\prime}\right)$ (Abragam, 1961; Cavanagh et al., 2007)

$$
\dot{\rho}=-\left[\overline{\widehat{H}_{l n t}(t)},\left[\overline{\widehat{H}_{l n t}\left(t-t^{\prime}\right)}, \rho\right]\right]=\rho^{\prime}
$$

\section{Interaction Hamiltonian for dipolar and CSA interactions}

The main magnetic interactions in relaxation in liquid state NMR are dipolar couplings and chemical shift anisotropy, which can be utilized to extract structural as well as dynamic information.

The Hamiltonian for the dipolar coupling is (Schwalbe et al., 2001)

$$
\begin{gathered}
\widehat{H}_{k l}^{D D}=b_{k l}\left\{3 \frac{1}{r_{k l}^{2}}\left(\hat{I}_{k} \cdot \vec{r}_{k l}\right)\left(\hat{I}_{l} \cdot \vec{r}_{k l}\right)-\hat{I}_{k} \hat{I}_{l}\right\} \\
=b_{k l} \sum_{q=-2}^{+2} F_{k l}^{(-q)}\left(\theta_{k l}, \phi_{k l}\right) \hat{A}_{k l}^{(q)}\left(\hat{I}_{k}, \hat{I}_{l}\right) \\
b_{k l}=-\mu_{0} \frac{\gamma_{k} \gamma_{l} \hbar}{4 \pi r_{k l}^{3}}
\end{gathered}
$$

Where $\gamma_{k}$ and $\gamma_{l}$ indicate the gyromagnetic ratio of the nuclei $k$ and $l, \hbar$ the reduced Planck constant, and $r_{k l}$ the distance between the two nuclei. The angles $\theta_{k l}$ and $\phi_{k l}$ describe the orientations of the vector $\vec{r}_{k l}$ with respect to the magnetic field. Using table $1, F_{k l}^{(-q)}\left(\theta_{k l}, \phi_{k l}\right)$, the time dependent spherical harmonics function and $\hat{A}_{k l}^{(q)}$ denote the second rank tensor operator can be substituted. 


\begin{tabular}{|c|c|c|c|c|}
\hline & $\begin{array}{l}\text { Tensor Operators for } \\
\text { the Dipolar Interaction } \\
b_{k l}=-\mu_{0} \frac{\gamma_{k} \gamma_{l} \hbar}{4 \pi r_{k l}^{3}}\end{array}$ & $\begin{array}{l}\text { Tensor Operators for } \\
\text { the CSA Interaction } \\
b_{k}=\frac{1}{3}\left(\sigma_{\|}-\sigma_{\perp}\right) \gamma_{k} B_{0}\end{array}$ & $\begin{array}{c}\text { Modified Spherical } \\
\text { Harmonics }\end{array}$ & Frequency \\
\hline & $\hat{A}_{k l}^{(q)}\left(\hat{I}_{k}, \hat{I}_{l}\right)$ & $\hat{A}_{k}^{(q)}\left(\hat{I}_{k}\right)$ & $F_{k}^{(q)}(\theta, \phi), F_{k l}^{(q)}(\theta, \phi)$ & $\omega_{q}$ \\
\hline-2 & $\sqrt{\frac{3}{8}} \hat{I}_{k}^{-} \hat{I}_{l}^{-}$ & - & $\sqrt{\frac{3}{2}} \sin ^{2} \theta \exp (+2 i \phi)$ & $\omega\left(\hat{I}_{k}\right)+\omega\left(\hat{I}_{l}\right)$ \\
\hline-1 & $\sqrt{\frac{3}{8}} \hat{I}_{k, z} \hat{I}_{l}^{-}$ & - & $\sqrt{6} \sin \theta \cos \theta \exp (+i \phi)$ & $\omega\left(\hat{I}_{l}\right)$ \\
\hline-1 & $\sqrt{\frac{3}{8}} \hat{I}_{k}^{-} \hat{I}_{l, z}$ & $\sqrt{\frac{3}{8}} \hat{I}_{k}^{-}$ & $\sqrt{6} \sin \theta \cos \theta \exp (+i \phi)$ & $\omega\left(\hat{I}_{k}\right)$ \\
\hline 0 & $\hat{I}_{k, z} \hat{I}_{l, z}$ & $\hat{I}_{k, z}$ & $3 \cos ^{2} \theta-1$ & 0 \\
\hline 0 & $\frac{1}{4}\left(\hat{I}_{k}^{+} \hat{I}_{l}^{-}+\hat{I}_{k}^{-} \hat{I}_{l}^{+}\right)$ & - & $3 \cos ^{2} \theta-1$ & $\omega\left(\hat{I}_{k}\right)-\omega\left(\hat{I}_{l}\right)$ \\
\hline 1 & $\sqrt{\frac{3}{8}} \hat{I}_{k}^{+} \hat{I}_{l, z}$ & $\sqrt{\frac{3}{8}} I_{k}^{+}$ & $\sqrt{6} \sin \theta \cos \theta \exp (-i \phi)$ & $-\omega\left(\hat{I}_{k}\right)$ \\
\hline 1 & $\sqrt{\frac{3}{8}} \hat{I}_{k, z} \hat{I}_{l}^{+}$ & - & $\sqrt{6} \sin \theta \cos \theta \exp (-i \phi)$ & $-\omega\left(\hat{I}_{l}\right)$ \\
\hline 2 & $\sqrt{\frac{3}{8}} \hat{I}_{k}^{+} \hat{I}_{l}^{+}$ & - & $\sqrt{\frac{3}{2}} \sin ^{2} \theta \exp (-2 i \phi)$ & $-\omega\left(\hat{I}_{k}\right)-\omega(\hat{I}$ \\
\hline
\end{tabular}

Table 1: Tensor Operators in the Rotating Frame and modified Spherical Harmonics for the dipolar and CSA Interaction (from Schwalbe et al., 2001). The calibration has been chosen such that

$\frac{1}{4 \pi} \int_{0}^{\pi} d \sin \theta \int_{0}^{2 \pi} d \phi F_{k}^{(q)}(\theta, \phi) F_{k l}^{(-q)}(\theta, \phi)=\frac{4}{5}$ and therefore $q$ independent.

The Hamiltonian for the CSA of nucleus $k$ in the principle axis frame (PAS) of chemical shift anisotropy tensor is given by ${ }^{[\mathrm{t} 3]}$

$$
\begin{gathered}
\widehat{H}_{k}^{C S A, P A S}=\gamma_{k} \sum_{i=x, y, z} B_{i} \hat{\hat{\sigma}}_{i i}^{k} \hat{I}_{k, i} \\
=\gamma_{k} \frac{\sigma_{x x}^{k}+\sigma_{y y}^{k}+\sigma_{z z}^{k}}{3} \vec{B}_{k}+\frac{1}{3} \gamma_{k}\left(\sigma_{x x}^{k}-\sigma_{z z}^{k}\right) \times\left[2 \hat{I}_{k, x} B_{x}-\hat{I}_{k, y} B_{y}-\hat{I}_{k, z} B_{z}\right] \\
+\frac{1}{3} \gamma_{k}\left(\sigma_{y y}^{k}-\sigma_{z z}^{k}\right) \times\left[2 \hat{I}_{k, y} B_{y}-\hat{I}_{k, x} B_{x}-\hat{I}_{k, z} B_{z}\right]
\end{gathered}
$$


Where $B_{i}$ indicate the components of the external static field $B_{0}$ in the PAS. In the laboratory frame (LF) the CSA Hamiltonian can be written by spherical harmonics $F_{k l}^{(-q)}\left(\theta_{k l}, \phi_{k l}\right)$ and spin tensor operators $\hat{A}_{k l}^{(q)}$. The corresponding expressions are listed in the table 1 and the Hamiltonian in the LF is given by (Schwalbe et al., 2001)

$$
\widehat{H}_{k}^{C S A, L F}=b_{k, x} \sum_{q=1}^{+1} F_{k}^{(q)}\left(\theta_{k, x}, \phi_{k, x}\right) \hat{A}_{k}^{(q)}\left(\hat{I}_{k}\right)+b_{k, y} \sum_{q=1}^{+1} F_{k}^{(q)}\left(\theta_{k, y}, \phi_{k, y}\right) \hat{A}_{k}^{(q)}\left(\hat{I}_{k}\right)
$$

With

$$
b_{k, x}=\frac{1}{3} \gamma_{k} B_{0}\left(\sigma_{x x}^{k}-\sigma_{z z}^{k}\right) \quad \text { and } \quad b_{k, y}=\frac{1}{3} \gamma_{k} B_{0}\left(\sigma_{y y}^{k}-\sigma_{z z}^{k}\right)
$$

And in case of an axially symmetric CSA tensor $\sigma_{x x}^{k}=\sigma_{y y}^{k}$, the equation is given by

$$
\begin{gathered}
\widehat{H}_{k}^{C S A, L F}=b_{k} \sum_{q=1}^{+1} F_{k}^{(q)}\left(\theta_{k}, \phi_{k}\right) \hat{A}_{k}^{(q)}\left(\hat{I}_{k}\right) \\
b_{k}=\frac{1}{3} \gamma_{k} B_{0}\left(\sigma_{\|}^{k}-\sigma_{\perp}^{k}\right)
\end{gathered}
$$

Where $\theta_{k}$ and $\phi_{k}$ are the polar angles of the $\mathrm{z}(\|)$ principal axis of the CSA tensor in the laboratory frame.

\section{Cross correlated relaxation (CCR)}

Cross correlated relaxation is originated from the interference of two anisotropic spin interactions which are averaged in a correlated way by the reorientation of the molecule in the solution (Schwalbe et al., 2001). Second rank tensors are used to describe the anisotropic interactions quantitatively. The CCR rate describes the orientation of two interactions with respect to each other and the dynamics of the investigated spin system in comparison with the 
overall correlation time of molecule. One of the ways to study the CCR in the solution NMR spectroscopy is to investigate the different line broadening of a multiplet signal.

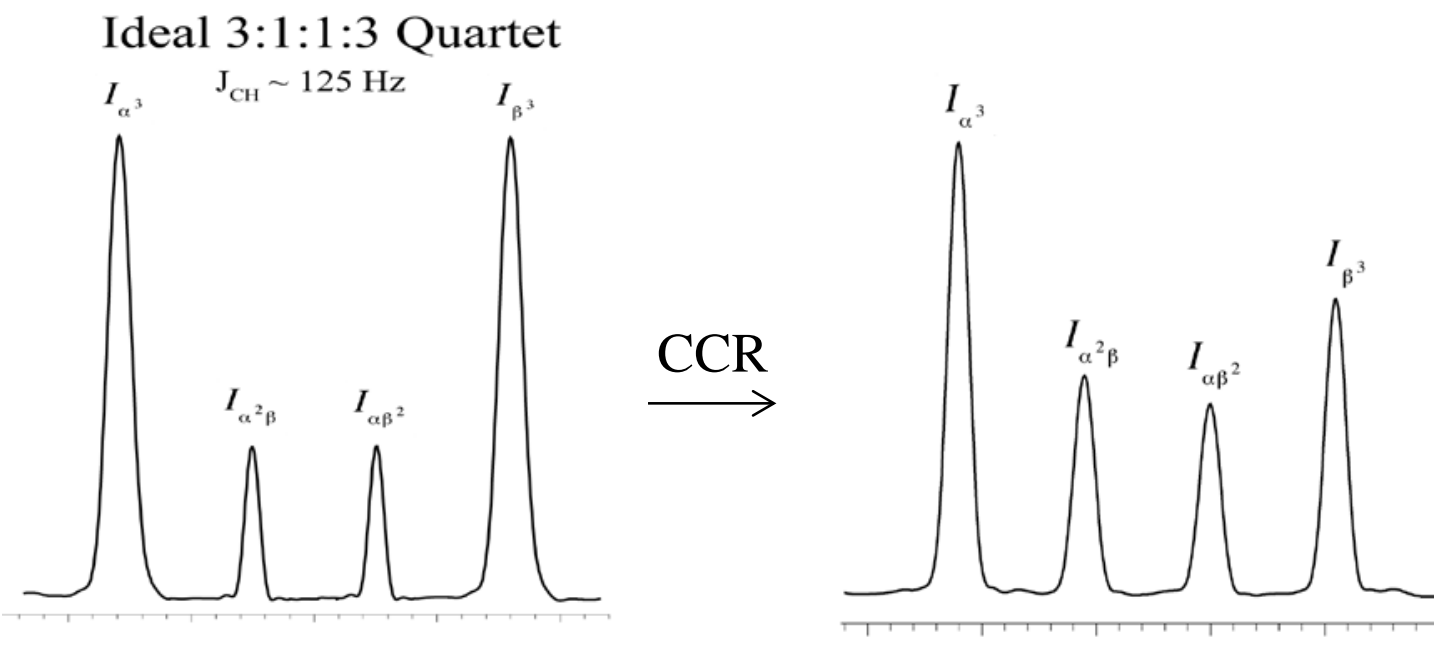

As explained in the previous section the main magnetic interactions which cause relaxation in the liquid NMR spectroscopy are dipolar coupling and CSA. The operator $\hat{\Gamma}_{\operatorname{spin}(i n), \operatorname{sp} i n(s)}^{i n t}$ represents the cross correlated relaxation between two interactions of two spins or bonds. For example $\hat{\Gamma}_{I_{k} I_{l}, I_{m} I_{n}}^{D D, D D}$ indicates the CCR between dipole-dipole interaction tensors for the vector connecting $I_{k}$ and $I_{l}$ as well as the vector connecting $I_{m}$ and $I_{n}$, respectively.

Using the Liouville von Neumann equation and double commutation rule we have

$$
\dot{\rho}=-i\left[\widehat{H}_{0}, \rho(t)\right]-\sum_{V, W} \widehat{\hat{\Gamma}}_{V, W}(\rho(t)-\rho(0))
$$

The general form of relaxation super operator is (Schwalbe et al., 2001)

$$
\widehat{\hat{\Gamma}}_{V, W} \rho(t)=b_{V} b_{W} \sum_{q}\left[\hat{A}_{V}^{(-q)},\left[\hat{A}_{W}^{(+q)}, \rho(t)\right]\right] j_{V, W}^{q}\left(\omega_{q}\right)
$$

where magnetic interactions as source of relaxation are referred by $V$ and $W$ indices. The term $j_{V, W}^{q}\left(\omega_{q}\right)$ refers to the spectral density function (Schwalbe et al., 2001).

$$
j_{V, W}^{q}\left(\omega_{q}\right)=\int_{0}^{\infty} d \tau \overline{F_{V}^{(q)}(t) F_{W}^{(-q)}(t+\tau)} \exp \left(-i \omega_{q} \tau\right)
$$


In the next chapter we show how to measure CCR rate of two dipolar interactions between two C-H bond vectors within a methyl group. Afterwards estimation of internal motion for methyl groups will be describes using this CCR rates. 


\section{Results and Discussion ${ }^{2}$}

\section{Methyl group cross-correlated relaxation}

Elimination of decoupling pulse on ${ }^{1} \mathrm{H}$ during ${ }^{13} \mathrm{C}$-chemical shift evolution in $2 \mathrm{D}$ constant time ${ }^{13} \mathrm{C}$, ${ }^{1} \mathrm{H}$ HSQC measurements leads to splitting of the methyl group ${ }^{13} \mathrm{C}$ signal into a quartet(Kay et al., 1992; Müller et al., 1987). The peaks in the quartet are separated by the scalar coupling constant $(J)$ for C-H bonds in a methyl group $\left(J_{C H} \sim 125 \mathrm{~Hz}\right)$. The four coherences are represented by each peak within the quartet, i.e. $\mathrm{C}_{x} H_{1 \alpha} H_{2 \alpha} H_{3 \alpha}$, $C_{x}\left(H_{1 \beta} H_{2 \alpha} H_{3 \alpha}+H_{1 \alpha} H_{2 \beta} H_{3 \alpha}+H_{1 \alpha} H_{2 \alpha} H_{3 \beta}\right), C_{x}\left(H_{1 \beta} H_{2 \beta} H_{3 \alpha}+H_{1 \alpha} H_{2 \beta} H_{3 \beta}+H_{1 \beta} H_{2 \alpha} H_{3 \beta}\right)$ and $C_{x}\left(H_{1 \beta} H_{2 \beta} H_{3 \beta}\right)$ and their intensity ratio $\left(I_{\alpha^{3}}: I_{\alpha^{2} \beta}: I_{\alpha \beta^{2}}: I_{\beta^{3}}\right)$ is 3:1:1:3 ignoring the contribution of relaxation (Kay et al., 1992; Tugarinov et al., 2003). With the involvement of the transverse relaxation rate ( $R_{2, I_{i}}$ ) (Kay and Torchia, 1991), the intensities can be expressed as:

$$
\begin{aligned}
& I_{\alpha^{3}} \propto 3 e^{-R_{2, I} \alpha_{\alpha^{3}} \Delta} \\
& I_{\alpha^{2} \beta} \propto e^{-R_{2, I} \alpha^{2} \beta^{3}} \\
& I_{\alpha \beta^{2}} \propto e^{-R_{2, I} \alpha_{\beta^{2}} \Delta} \\
& I_{\beta^{3}} \propto 3 e^{-R_{2, I \beta^{3}} \Delta},
\end{aligned}
$$

where $\Delta$ is the length of the constant time period.

By considering the dipolar coupling and chemical shift anisotropy (CSA), $R_{2, I_{i}}$ can be written as (Liu et al., 2003; Kay and Torchia, 1991):

\footnotetext{
${ }^{2}$ This part was done partly by the author and partly by Dr. T. Michael Sabo. All measurements, processing, spectral analysis, calculation of CCR rates and corresponding errors were done by author. Extraction of order parameters $\left(\mathrm{S}^{2}\right)$ and their error analysis were done together. All temperature dependence analysis, calculation of thermal coefficients and corresponding error estimation were done by Dr. T. M. Sabo.
} 


$$
\begin{aligned}
& R_{2, I_{\alpha^{3}}}=\lambda+3 \sigma+2 \eta \\
& R_{2, I_{\alpha^{2} \beta}}=\lambda-\sigma+\frac{2}{3} \eta \\
& R_{2, I_{\alpha \beta^{2}}}=\lambda-\sigma-\frac{2}{3} \eta \\
& R_{2, I_{\beta^{3}}}=\lambda+3 \sigma-2 \eta
\end{aligned}
$$

where $\lambda$ is the rate of the autorelaxation and $\sigma$ and $\eta$ are the rate of the CCR between dipolar couplings of two $\mathrm{CH}$ bonds and between dipolar coupling of $\mathrm{CH}$ and $\mathrm{CSA}$ of the ${ }^{13} \mathrm{C}$ nucleus, respectively. Using the intensities of the quartet, the CCR rate between dipolar couplings of two CH bonds can be calculated from the experimental intensities, (Liu et al., 2003)

$$
\sigma_{o b s}=\frac{1}{8 \Delta} \ln \frac{9 I_{\alpha^{2} \beta} I_{\alpha \beta^{2}}}{I_{\alpha^{3}} I_{\beta^{3}}} \text {. }
$$

Here we report the temperature dependence of $\sigma_{o b s}$ for the methyl groups of uniformly ${ }^{15} \mathrm{~N}$, ${ }^{13} \mathrm{C}$-labeled wild type human ubiquitin extracted from a series of proton coupled 2D constant time ${ }^{13} \mathrm{C},{ }^{1} \mathrm{H}$ HSQC measurements at fourteen temperatures: 275, 278, 281, 283, 286, 288, 291, 293, 296, 298, 301, 303, 305, and 308 K. Ubiquitin contains 50 methyl groups residing in 30 residues. Despite the increase in $\tau_{c}$ accompanied with lowering the temperature at 275 $\mathrm{K}$, it is clear from the spectrum at this temperature (see Figure 1), the peaks in the highlighted quartets are well resolved. Furthermore, even at $275 \mathrm{~K}$, a significant amount of motion is considerable for the methyl groups of ubiquitin, especially for L $8 \delta 1$ whose quartet approaches the ideal intensity ratio of 3:1:1:3.

For almost 50\% of all methyl groups in ubiquitin, equation (3) was employed to calculate $\sigma_{o b s}$ at each of the fourteen temperatures. The results are compiled in appendix I. For the remaining 28 methyl groups, either spectral crowding becomes problematic due to the chemical shift differences between methyl group carbons being similar to $J_{C H}, 2 J_{C H}$, or $3 J_{C H}$ (Liu et al., 2003) and/or strong coupling is active between the $\delta$ and $\gamma$ carbons in leucine as reported for L15 $\delta 1, \mathrm{~L} 43 \delta 1, \mathrm{~L} 50 \delta 1, \mathrm{~L} 56 \delta 1$, and L69 $\delta 2$ (Zhang et al., 2006). Since the data 
should be covered in all temperature points, we only analyzed methyl groups whose quartets were not overlapped with other methyl group quartets over the entire temperature range (275 $\mathrm{K}$ to $308 \mathrm{~K})$.

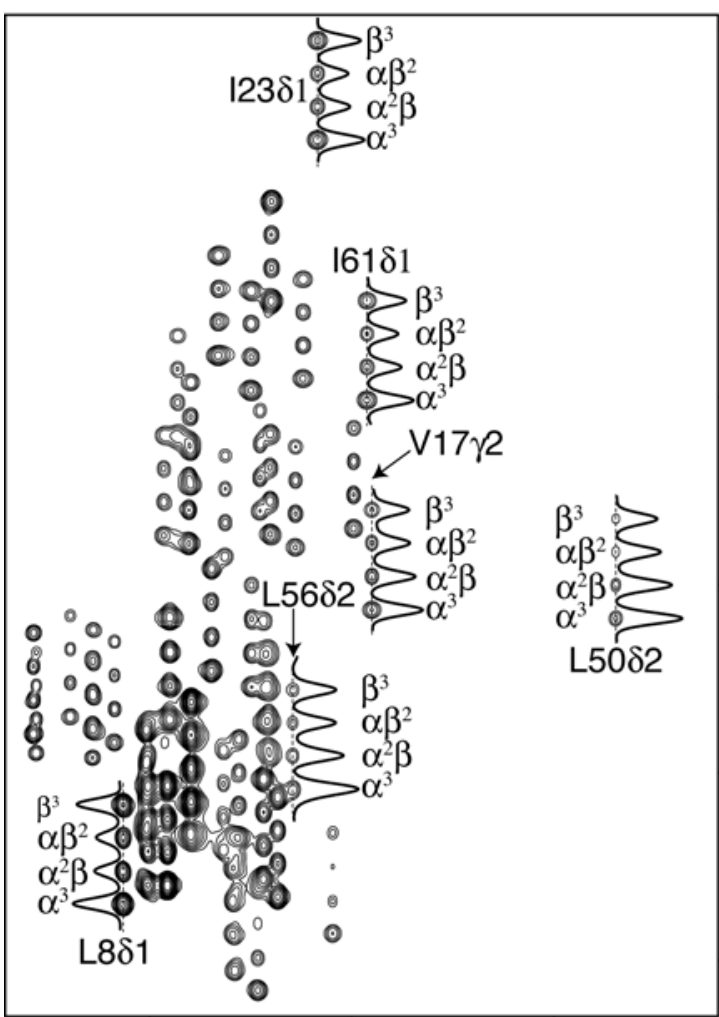

$1.00 \quad{ }^{1} \mathrm{H}(\mathrm{ppm}) \quad 0.00$
8.0

Figure 1. Constant time ${ }^{13} \mathrm{C},{ }^{1} \mathrm{H}$ HSQC spectrum of uniformly ${ }^{15} \mathrm{~N},{ }^{13} \mathrm{C}$-labeled human wild type ubiquitin measured at frequency of $700 \mathrm{MHz}$ (for ${ }^{1} \mathrm{H}$ ) and a temperature of $275 \mathrm{~K}$. The ubiquitin concentration was 3.6 $\mathrm{mM}$ in $90 \% / 10 \% \mathrm{H}_{2} \mathrm{O} / \mathrm{D}_{2} \mathrm{O}$, with $50 \mathrm{mM}$ sodium phosphate at $\mathrm{pH} 6.8,100 \mathrm{mM} \mathrm{NaCl}$ and $0.1 \% \mathrm{NaN}_{3}$. The constant time length and INEPT delays were set to 27.8 and $2 \mathrm{~ms}$, respectively. The spectrum was acquired with 1024 and 128 complex points in the direct $\left(t_{2}\right)$ and indirect $\left(t_{1}\right)$ dimensions, respectively, with 8 scans per $t_{1}$ increment. The $t_{1}, \max$ and $t_{2, \max }$ were set to $24.3 \mathrm{~ms}$ and $113 \mathrm{~ms}$, respectively. Frequency discrimination for the indirectly detected dimension was done with the States-TPPI scheme.

24.0 The raw data was processed with NMRPipe software. ${ }^{44} 1 \mathrm{D}$ slices of selected quartets are shown together with the corresponding coherences for each peak in the multiplet 28.0 (Sabo et al., 2012)

It is clear from the range of $\sigma_{o b s}$ at each temperature that the methyl groups exist in a wide array of environments. Changes in the mobility of the methyl groups contribute to the apparent differences in $\sigma_{o b s}$. It should be considered that studying the temperature dependence of methyl group dynamics with deuterium relaxation studies (Muhandiram et al., 1995; Liao et al., 2012; Millet et al., 2002; Lee et al., 1999), enables many more methyl groups to be analyzed due to significantly less spectral overlap, despite the very long time demand for data collection. Nevertheless, various techniques for specific labeling of methyl groups (Goto et al., 1999; Godoy-Ruiz et al., 2010; Ruschak et al., 2010), which now even includes 
stereospecific selection of methyl groups (Gans et al., 2010), we assume that this approach might be applicable to proteins larger than ubiquitin.

\section{Quantification of methyl group dynamics in ubiquitin}

Methyl group order parameter ( $S_{\text {axis }}^{2}$ ) can be used to extracted from experimental CCR rates ( $\left.\sigma_{o b s}\right)$ by comparing them with the theoretical value of the CCR $\left(\sigma_{\text {rigid }}\right)$ in the absence of local motions (Liu et al., 2003; Kay and Torchia, 1991; Lee et al., 2006):

$$
\begin{gathered}
S_{a x i s}^{2}=\frac{\sigma_{o b s}}{\sigma_{\text {rigid }}} \\
\sigma_{\text {rigid }}=\frac{1}{45}\left(\frac{\mu_{0} h \gamma_{H} \gamma_{C}}{8 \pi^{2} r_{C H}^{3}}\right)^{2}\left(2 \tau_{c}+\frac{3 \tau_{c}}{2\left(1+\left(\omega_{c} \tau_{c}\right)^{2}\right)}\right)
\end{gathered}
$$

where $\mu_{0}$ is the permeability of a vacuum, $h$ is the Planck constant, $\gamma_{H}$ and $\gamma_{C}$ are the gyromagnetic ratios of ${ }^{1} \mathrm{H}$ and ${ }^{13} \mathrm{C}$, respectively, $r_{C H}$ is the $\mathrm{CH}$ bond length, $\omega_{C}$ is the Larmor frequency of ${ }^{13} \mathrm{C}$ and $\tau_{c}$ is the rotational correlation time. $S_{\text {axis }}^{2}$ is a dimensionless quantity for describing the magnitude of mobility for the methyl group (Kay and Torchia, 1991). Values for $S_{\text {axis }}^{2}$ vary between 0 and 1 , where 1 regards to a rigid methyl group and zero represents unrestricted local motion.

For determination of $\sigma_{\text {rigid }}$ with equation (5), the methyl group C-H bond length $\left(r_{C H}\right)$ was set to 1.095 angstroms, tetrahedral geometry assumed and the $\tau_{c}$ of ubiquitin at each temperature is reported in appendix I. Using $\sigma_{\text {rigid, }}$, we calculated $S_{a x i s}^{2}$ with equation (4) for all fourteen temperatures, presented in the appendix I. Figure 2 shows the correlation of calculated $S_{a x i s}^{2}$ at a selected set of temperatures. From the figure a high degree of correlation for $S_{a x i s}^{2}$ over these temperatures is apparent. For all pair-wise combinations of $S_{a x i s}^{2}$, the Pearson correlation coefficient is $r \geq 0.98$. Due to the linear dependence of $S_{a x i s}^{2}$ on $\mathrm{T}$, the high 
correlation between $S_{\text {axis }}^{2}$ at every temperature suggests that uncertainties emerging from each experimental measurement are consistent over the whole data set. Figure 3A illustrates the correlation plot of $S_{\text {axis }}^{2}$ values from CCR measurements versus $S_{a x i s}^{2}$ calculated from the rates of multiple spin coherences involving ${ }^{2} \mathrm{H}$ in the methyl group, both at $303 \mathrm{~K}$

(Lee et al., 1999). Figure 3B reveals the correlation plot of the $S_{a x i s}^{2}$ from this study at $301 \mathrm{~K}$ versus $S_{\text {axis }}^{2}$ extracted from methyl group ${ }^{13} \mathrm{C}$ spin-lattice $\left(T_{1}\right)$ relaxation rates and $\sigma$ modulated by the one bond C-H coupling constant $\left(J_{C H}\right)$ measured at $300 \mathrm{~K}$ (Zhang et al., 2006). The Pearson correlation coefficient is high for both comparisons, 0.96 and 0.97 respectively. The high correlation between $S_{\text {axis }}^{2}$ obtained by three independent methodologies together with the high correlation for $S_{a x i s}^{2}$ over the entire temperature range provides strong confirmation that the temperature dependence of $S_{a x i s}^{2}$ can be studied using the present method. It should be noted that deviations from the diagonal is most likely an evidence for small differences in experimental setup, such as sample conditions, slight temperature variations, and/or uncertainty in measurements. Most importantly, the total amount of time required to obtain this information is efficiently reduced. With approaches involving ${ }^{2} \mathrm{H}$ labeling of methyl groups (Muhandiram et al., 1995; Liao et al., 2012; Millet et al., 2002), $R_{1}$ and $R_{2}$ relaxation rates are typically measured in order to calculate $S_{a x i s}^{2}$ at one temperature. As for $\sigma$ modulated by the one bond C-H coupling constant $\left(J_{C H}\right), S_{a x i s}^{2}$ determined at one temperature needs a series of $2 \mathrm{D}$ constant time ${ }^{13} \mathrm{C},{ }^{1} \mathrm{H}$ HSQC experiments with increasing delay times (Zhang et al., 2006). However, with the introduced method, one 2D constant time ${ }^{13} \mathrm{C},{ }^{1} \mathrm{H}$ HSQC measurement is needed to acquire the same amount of information at one temperature point.

Finally, the time-scale of motion covered by $S_{a x i s}^{2}$ is faster than the $\tau_{c}$ of ubiquitin, describing ps to ns dynamics. Lakomek et al (2009) has determined the methyl group RDC- 
based order parameters $S_{R D C}^{2}$ for ubiquitin, which encompasses time-scales from ps to ms. By comparison of two sets of order parameters at $308 \mathrm{~K}$, the average values of $S_{\text {axis }}^{2}$ and $S_{R D C}^{2}$ are $0.59 \pm 0.21$ and $0.43 \pm 0.25$, respectively, with a Pearson correlation coefficient of $r=0.84$. It is obvious that additional dynamics on a slower time-scale for the methyl groups exist than the motion encapsulated by $S_{a x i s}^{2}$.

\section{Analysis of the temperature dependence of $S_{a x i s}^{2}$ in ubiquitin}

Temperature dependencies of $S_{a x i s}^{2}$ can be quantified by the thermal coefficient $\kappa$ was taken from the linear fit of $S_{a x i s}^{2}$ versus temperature $\left(\kappa=\frac{d S_{a x i s}^{2}}{d T}\right)$ (Lee et al., 2002; ) Table 1 and Figure 4 show the results of the fitting procedure. Decrease of $S_{a x i s}^{2}$ as a general trend is observed in the temperature range from 275 to $308 \mathrm{~K}$. The average value of $\kappa$ is $-(2.9 \pm 1.5) \times$ $10^{-3} \mathrm{~K}^{-1}$ which is in good agreement with the value of $-(2.6 \pm 1.1) \times 10^{-3} \mathrm{~K}^{-1}$ reported previously for ubiquitin over the larger temperature range of 278 to $328 \mathrm{~K}$ (Song et al., 2002). In addition, similar trends in the deviation of $S_{a x i s}^{2}$ with temperature can also be seen for calmodulin bound to a peptide (Lee et al., 2002). Figure 5A presents the distribution of $\kappa$ within ubiquitin (1UBQ: Vijayakumar et al., 1987). The maximum $\kappa$ values are grouped around I6181, spatially near the N-terminus of ubiquitin, and progressively decrease toward the C-terminus of the protein.

The characteristic thermal coefficient $\Lambda=d \ln \left(1-\sqrt{S_{a x i s}^{2}}\right) / d \ln T$ correlates the temperature dependencies of the generalized order parameter, in this case $S_{a x i s}^{2}$, to the characteristic temperature ( $T^{*}$ ) (Mandel et al., 1996; Vugmeyster et al., 2002). The term $T^{*}$ is a description for the density of thermally accessible conformational states for the bond vector, here methyl axis of symmetry (Mandel et al., 1996). Table 1 lists the results for the determination of $\Lambda$, 
which correlates with $\kappa($ Pearson coefficients of $r=0.7)$. The average value of $\Lambda(3.4 \pm 2.4$ compared to $2.3 \pm 1.0$ previously reported for ubiquitin over the larger temperature range of 278 to $328 \mathrm{~K}$ (Song et al., 2007)) reveals significant contributions from rotameric jumps within the side chains (Lee and Wand, 2001). In Figure 5B depicts the distribution of $\Lambda$ on the structure of ubiquitin. Similar to the magnitude of $\kappa$, the largest values of $\Lambda$ are clustered in the $\mathrm{N}$-terminal region of ubiquitin. A successive decline of $\Lambda$ towards the C-terminal region is observed.
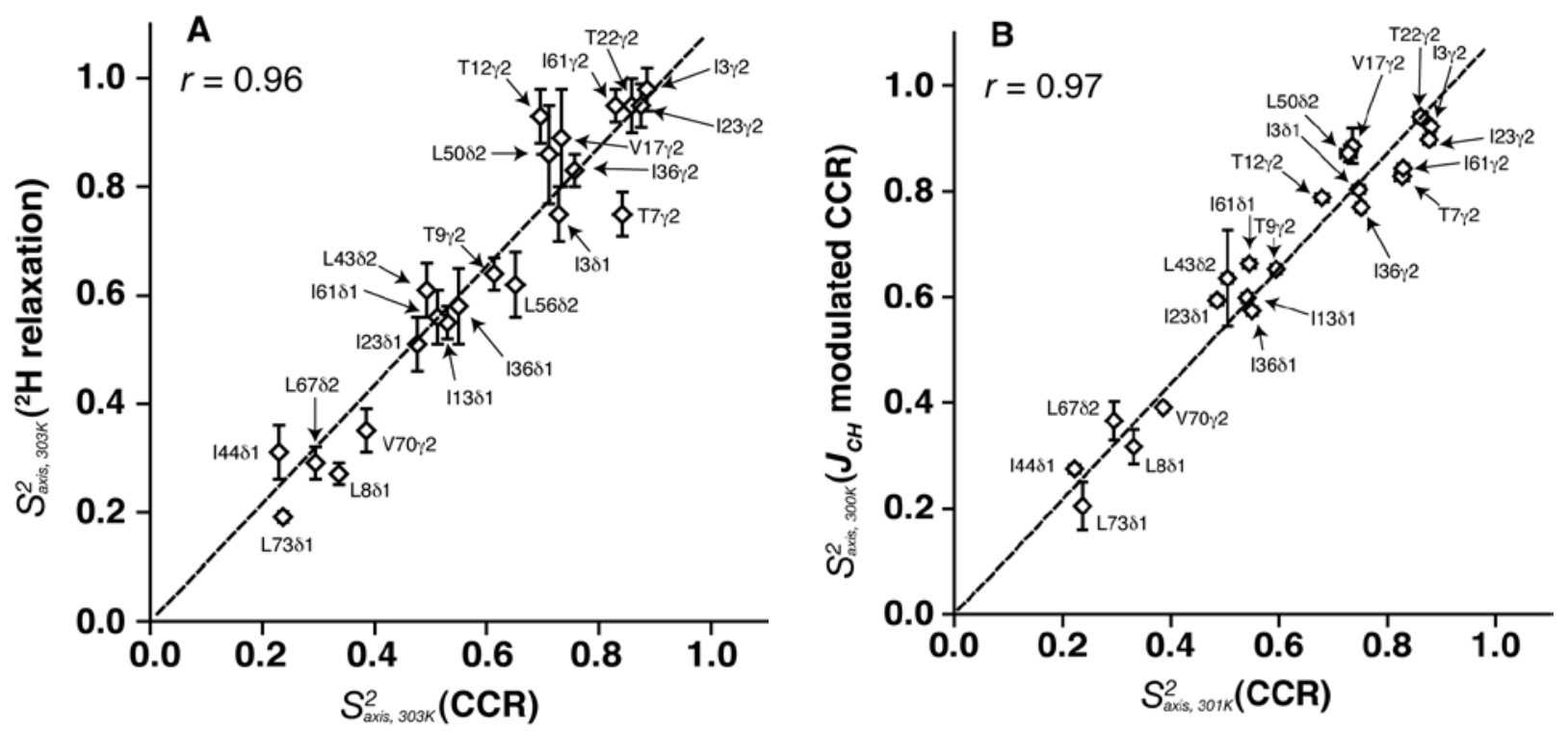

Figure 3. Comparison of $S_{a x i s}^{2}$ in ubiquitin obtained from different methods. (A) Correlation between $S_{a x i s}^{2}$ determined from the present study using dipolar cross-correlated relaxation between $\mathrm{CH}$ bonds in the methyl group versus $S_{\text {axis }}^{2}$ calculated from the rates of multiple spin coherences involving ${ }^{2} \mathrm{H}$ in the methyl group. ${ }^{26}$ In both cases, $S_{\text {axis }}^{2}$ was estimated at $303 \mathrm{~K}$.

Correlation between $S_{\text {axis }}^{2}$ determined at $301 \mathrm{~K}$ from the present study versus $S_{\text {axis }}^{2}$ calculated at $300 \mathrm{~K}$ from methyl group ${ }^{13} \mathrm{C}$ spin-lattice $\left(\mathrm{T}_{1}\right)$ relaxation rates and dipolar cross-correlated relaxation modulated by the one bond C-H coupling constant $\left(J_{C H}\right){ }^{21}$ In both figures, the dashed lines illustrate the best fit to the data. The error bars are shown in both dimensions. The Pearson correlation $r$ is also designated in the figures (Sabo et al., 2012) 


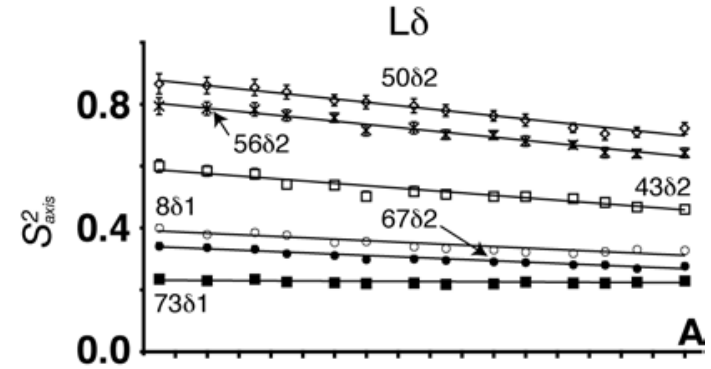

$\mid \delta 1$

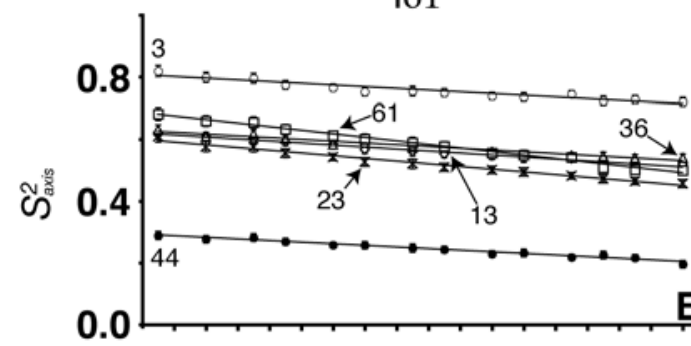

$1 \gamma 2$
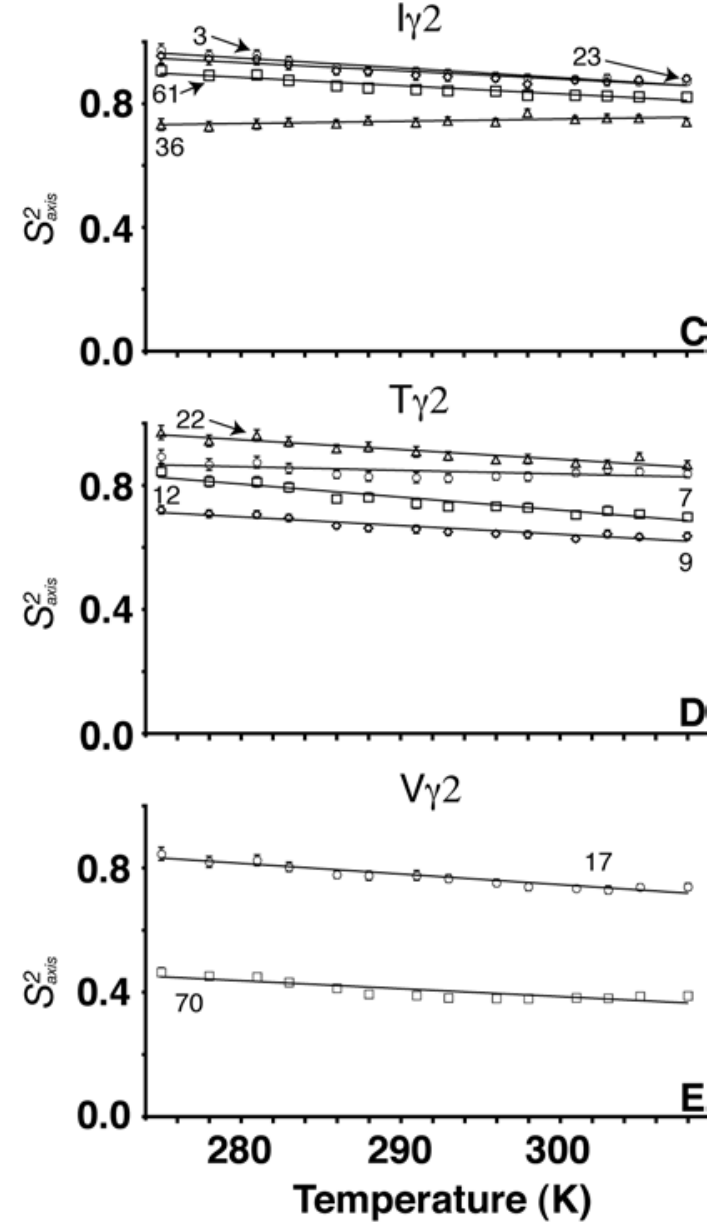

Figure 4. Temperature dependence of order parameters $\left(S_{a x i s}^{2}\right)$ in ubiquitin ( $\left.\kappa=\frac{d S_{a x i s}^{2}}{d T}\right)$. All data were fit with a linear regression line in order to achieve $\kappa$ (solid line). ${ }^{15}$ The error bars are smaller than the symbol size for many of the data points represented here.

(A) Representation of the temperature dependence of $S_{\text {axis }}^{2}$ for the following leucine $\delta$ methyl groups: $8 \delta 1(\circ)$, $43 \delta 2(\square), 50 \delta 2(\diamond), 56 \delta 2(\times), 67 \delta 2(\bullet)$, and $73 \delta 1(\bullet)$.

(B) Representation of the temperature dependence of $S_{\text {axis }}^{2}$ for the following isoleucine $\delta 1$ methyl groups: 3 (॰), $13(\diamond), 23(\times), 36(\diamond), 44(\bullet)$, and $61(\square)$. (C) Representation of the temperature dependence of $S_{\text {axis }}^{2}$ for the following isoleucine $\gamma 2$ methyl groups: $3(\circ), 23(\diamond)$, $36(\triangle)$, and $61(\square)$. (D) Representation of the temperature dependence of $S_{\text {axis }}^{2}$ for the following threonine $\gamma 2$ methyl groups: $7(\circ), 9(\diamond), 12(\square)$, and $22(\triangle)$. (E) Representation of the temperature dependence of $S_{\text {axis }}^{2}$ for the following valine $\gamma 2$ methyl groups: 17 (०) and 70 (口) (From Sabo et al., 2012). 
Table 2. Thermal coefficients extracted from methyl group order parameters ( $\left.S_{a x i s}^{2}\right)$ within ubiquitin $^{a}$

\begin{tabular}{|c|c|c|c|}
\hline & $\kappa\left(\mathrm{x} 10^{-3} \mathrm{~K}^{-1}\right)^{b}$ & $\Lambda^{c}$ & $C_{p}\left(\mathrm{~J} \mathrm{~K}^{-1} \mathrm{~mol}^{-1}\right)^{d}$ \\
\hline $\mathrm{I} 3 \gamma 2$ & $-2.9 \pm 0.4$ & $7.5 \pm 1.4$ & $52.7 \pm 11.9^{e}$ \\
\hline I3 $\delta 1$ & $-2.5 \pm 0.4$ & $3.2 \pm 0.5$ & $27.3 \pm 4.5$ \\
\hline $\mathrm{T} 7 \gamma 2$ & $-0.8 \pm 0.5$ & $1.0 \pm 0.8$ & $8.5 \pm 7.9$ \\
\hline $\mathrm{L} 8 \delta 1$ & $-2.3 \pm 0.2$ & $1.4 \pm 0.1$ & $13.1 \pm 1.1$ \\
\hline Т9 2 & $-3.0 \pm 0.3$ & $2.7 \pm 0.1$ & $23.4 \pm 2.8$ \\
\hline $\mathrm{T} 12 \gamma 2$ & $-4.4 \pm 0.4$ & $4.8 \pm 0.4$ & $41.5 \pm 4.9$ \\
\hline $\mathrm{I} 13 \delta 1$ & $-3.2 \pm 0.3$ & $2.5 \pm 0.2$ & $22.4 \pm 2.3$ \\
\hline $\mathrm{V} 17 \gamma 2$ & $-3.1 \pm 0.4$ & $4.0 \pm 0.4$ & $34.7 \pm 4.3$ \\
\hline $\mathrm{T} 22 \gamma 2$ & $-3.3 \pm 0.5$ & $8.1 \pm 0.4$ & $59.7 \pm 14.1^{e}$ \\
\hline I23 2 & $-2.3 \pm 0.4$ & $5.5 \pm 1.1$ & $43.9 \pm 11.4^{f}$ \\
\hline $\mathrm{I} 23 \delta 1$ & $-4.3 \pm 0.4$ & $3.1 \pm 0.2$ & $27.6 \pm 2.6$ \\
\hline I36 2 & $0.1 \pm 0.4$ & $-0.8 \pm 0.3$ & $-6.9 \pm 3.7$ \\
\hline I36 1 & $-2.8 \pm 0.3$ & $2.2 \pm 0.2$ & $19.8 \pm 2.4$ \\
\hline $\mathrm{L} 43 \delta 2$ & $-3.6 \pm 0.4$ & $2.6 \pm 0.2$ & $23.4 \pm 2.4$ \\
\hline $\mathrm{I} 44 \delta 1$ & $-2.6 \pm 0.3$ & $1.5 \pm 0.2$ & $14.8 \pm 1.7$ \\
\hline L50 2 & $-5.4 \pm 0.6$ & $7.5 \pm 0.6$ & $64.5 \pm 7.7$ \\
\hline L56 22 & $-5.2 \pm 0.5$ & $5.7 \pm 0.4$ & $49.3 \pm 4.9$ \\
\hline $\mathrm{I} 61 \gamma 2$ & $-2.5 \pm 0.3$ & $4.7 \pm 0.6$ & $39.5 \pm 6.6$ \\
\hline $\mathrm{I} 61 \delta 1$ & $-5.7 \pm 0.4$ & $4.6 \pm 0.3$ & $40.4 \pm 3.0$ \\
\hline $\mathrm{L} 67 \delta 2$ & $-2.1 \pm 0.2$ & $1.2 \pm 0.1$ & $11.8 \pm 1.2$ \\
\hline V70 2 & $-2.3 \pm 0.3$ & $1.4 \pm 0.1$ & $13.4 \pm 1.5$ \\
\hline L73 11 & $0.0 \pm 0.1$ & $0.2 \pm 0.1$ & $1.6 \pm 0.7$ \\
\hline Average & $-2.9 \pm 1.5$ & $3.4 \pm 2.4$ & $28.5 \pm 18.9$ \\
\hline
\end{tabular}

${ }^{a}$ All errors were calculated from 500 Monte Carlo simulation runs.

${ }^{b}$ The temperature dependencies of $S_{\text {axis }}^{2}\left(\kappa=\frac{d S_{\text {axis }}^{2}}{d T}\right)$ were achieved from the slope of a linear fit of $S_{a x i s}^{2}$ versus temperature (T) (Lee et al., 2002).

${ }^{c}$ The characteristic thermal coefficient $\Lambda$ was estimated from the slope of a linear fit of $\ln \left(1-\sqrt{S_{\text {axis }}^{2}}\right)$ versus $\ln T$ (Vugmeyster et al., 2002).

${ }^{d}$ The heat capacities $\left(C_{p}\right)$ were calculated from the slope of a linear fit of the conformational entropy ( $S_{\text {conf }}$ ) versus $\ln T$ (Privalov and Gill, 1988), where $S_{\text {conf }}=k_{B} N_{A}\left\{\ln \pi\left[3-\sqrt{1+8 \sqrt{S_{\text {axis }}^{2}}}\right]\right\}$. (Yang and Kay, 1996 and 1997)Constants $k_{B}$ and $N_{A}$ are the Boltzmann constant and Avogadro's number, respectively.

${ }^{e}$ For these methyl groups, only $S_{\text {axis }}^{2}$ from temperatures between 283 and $308 \mathrm{~K}$ were taken for the fitting procedure due to the requirement of $S_{\text {axis }}^{2}<0.95$ for determining $S_{\text {conf }}$. (Yang and Kay, 1996)

${ }^{f}$ For this methyl group, only $S_{\text {axis }}^{2}$ from temperatures between 278 and $308 \mathrm{~K}$ were taken for the fitting procedure due to the requirement of $S_{\text {axis }}^{2}<0.95$ for determining of $S_{\text {conf }}$. (Yang and Kay, 1996) 


\section{Determination of the $C_{p}$ from the temperature dependence of the conformational entropy}

It has been shown that the equation, $S_{\text {conf }}(j)=-k_{B} \int_{V} p(q) \ln p(q) d V$, can be used for calculation of conformational enthropy, where $p(q)=p(\theta, \phi)$ and $d V=\sin \theta d \theta d \phi$. Considering the model free formalism for order parameter (Lipari and Szabo, 1982a, b) the NMR relaxation parameters can be deciphered in terms of generalized order parameter, $S^{2}$. The value of order parameter for a bond vector motion is defined as

$$
S^{2}=\frac{4 \pi}{5} \sum_{m=-2}^{2}\left|\sum_{i} p\left(\theta_{i}, \phi_{i}\right) Y_{2 m}\left(\theta_{i}, \phi_{i}\right)\right|^{2}
$$

Where $Y_{2 m}\left(\theta_{i}, \phi_{i}\right)$ is the second rank spherical harmonic function and $\left(\theta_{i}, \phi_{i}\right)$ are the polar angels of the ith orientation of the vector in a molecular reference frame. This equation can be rewritten assuming a normalized probability distribution function, $p(q)$,

$$
S^{2}=\frac{4 \pi}{5} \sum_{m=-2}^{2}\left|\int_{V} p(q) Y_{2 m}(q) d V\right|^{2}
$$

Where $p(q)=p(\theta, \phi), Y_{2 m}(q)=Y_{2 m}(\theta, \phi)$ and $d V=\sin \theta d \theta d \phi$. As the order parameter like conformational entropy depends only on the probability distribution function, it is possible to interconnect these two parameters. There are many models for description of probability density function based on the type of motion of bond vector and the corresponding potential energy (Yang and Kay, 1996). The most widely used model for internal motion is the diffusion-in-a-cone (Brainard and Szabo, 1981).

$$
p(q)=\left\{\begin{array}{lll}
\frac{1}{\left\{\phi_{0}\left(1-\cos \theta_{0}\right\}\right.} & \text { for } 0 \leq \theta \leq \theta_{0}, & 0 \leq \phi \leq 2 \pi \\
0 & \text { for } & \theta>\theta_{0}
\end{array}\right.
$$

Using an extension of this model (Yang and Kay, 1996) by considering the free diffusion within the boundary $0 \leq \phi \leq \phi_{0}\left(\mathrm{p}(\mathrm{q})=0 ; \phi>\phi_{0}\right)$, the values for $S^{2}$ and $S_{\text {conf }}$ can be written as

$$
S_{\text {conf }}=k_{B} \ln \left\{\phi_{0}\left(1-\cos \theta_{0}\right)\right\}
$$




$$
\begin{aligned}
S^{2}= & \frac{1}{4} \cos ^{2} \theta_{0}\left(1+\cos \theta_{0}\right)^{2}+\frac{2}{3} \frac{\sin ^{6} \theta_{0}\left(1-\cos \phi_{0}\right)}{\left(1-\cos \theta_{0}\right)^{2} \phi_{0}^{2}}+ \\
& \frac{3}{8} \frac{\left(\cos \theta_{0}-\left(\frac{1}{3}\right) \cos ^{3} \theta_{0}-\left(\frac{2}{3}\right)\right)^{2}\left(1-\cos 2 \phi_{0}\right)}{\left(1-\cos \theta_{0}\right)^{2} \phi_{0}^{2}}
\end{aligned}
$$

Now regarding this model $S_{\text {conf }}$ and $S^{2}$ can be connected analytically as

$$
S_{\text {conf }}=k_{B} \ln \pi\left[3-\sqrt{\left(1+8 \sqrt{S^{2}}\right)}\right], \quad 0 \leq S^{2} \leq 1
$$

It should be noted that for order parameters in range of $0 \leq S^{2} \leq \frac{1}{64}$ there are two $S_{\text {conf }}$ values to be calculated (Yang and Kay, 1996) as

$$
S_{\text {conf }}=k_{B} \ln \pi\left[3 \pm \sqrt{\left(1+8 \sqrt{S^{2}}\right)}\right]
$$

As all order parameters reported in this work are larger than 1/64 we ignore this case.

Using an approximation that describes the motion of axis of symmetry of methyl group by diffusion-in-a-cone model (Tzeng and Kalodimos, 2012), the molar conformational entropy $\left(S_{\text {conf }}\right)$ can be related to $S_{a x i s}^{2}$,

$$
S_{\text {conf }}=k_{B} N_{A}\left\{\ln \pi\left[3-\sqrt{1+8 \sqrt{S_{a x i s}^{2}}}\right]\right\},
$$

where $k_{\mathrm{B}}$ is the Boltzmann constant and $N_{\mathrm{A}}$ is Avogadro's number (Yang and Kay, 1996). The heat capacity $\left(C_{p}\right)$ calculated from the temperature dependence of $S_{\text {conf }}$ is defined as (Privalov and Gill, 1988)

$$
C_{p}=\frac{d S_{\text {conf }}}{d \ln T}
$$

$C_{p}$ for the methyl groups in ubiquitin are presented in Table 2 for the temperature range of 275 to $308 \mathrm{~K}$. For 22 methyl groups in ubiquitin, an average $C_{p}$ of $28.5 \pm 18.9 \mathrm{~J} \mathrm{~K}^{-1} \mathrm{~mol}^{-1}$ is calculated using presented method. In another study for the drkN SH3 domain, similar values for the methyl group $C_{p}$ were obtained: $17 \pm 12 \mathrm{~J} \mathrm{~K}^{-1} \mathrm{~mol}^{-1}$ and $33 \pm 23 \mathrm{~J} \mathrm{~K}^{-1} \mathrm{~mol}^{-1}$ for the 
temperature intervals 287 to $303 \mathrm{~K}$ and 278 to $287 \mathrm{~K}$, respectively (Yang et al., 1997). Using data measured by differential scanning calorimetry, the global $C_{p}$ for ubiquitin was $\sim 12.6 \mathrm{~kJ}$ $\mathrm{K}^{-1} \mathrm{~mol}^{-1}$ at $298 \mathrm{~K}$ (Wintrode et al., 1995). In this dissertation I report, the summation of all the individual methyl groups $C_{p}$ equals $626 \mathrm{~J} \mathrm{~K}^{-1} \mathrm{~mol}^{-1}$. This value, however, does not include the contributions of 28 additional methyl groups where $C_{p}$ could not be extracted, these results suggest that the total methyl group $C_{p}$ makes an approximately $10 \%$ contribution to the global $C_{p}$ of ubiquitin. It should be considered that a majority $C_{p}$ can be determined from the primary sequence of proteins (Gomez et al., 1995). Since atoms of the methyl groups in ubiquitin are $16 \%$ (200 out of 1231) of all atoms, the $~ 10 \%$ contribution for the methyl groups to the total heat capacity of ubiquitin might be a reasonable estimate. Figure 5C illustrates the distribution of $C_{p}$ in ubiquitin. The methyl groups with largest values of $C_{p}$, similar to $\kappa$ and $\Lambda$, are accommodated spatially near the $\mathrm{N}$-terminus of the protein, which is the part of the hydrophobic core of ubiquitin. 

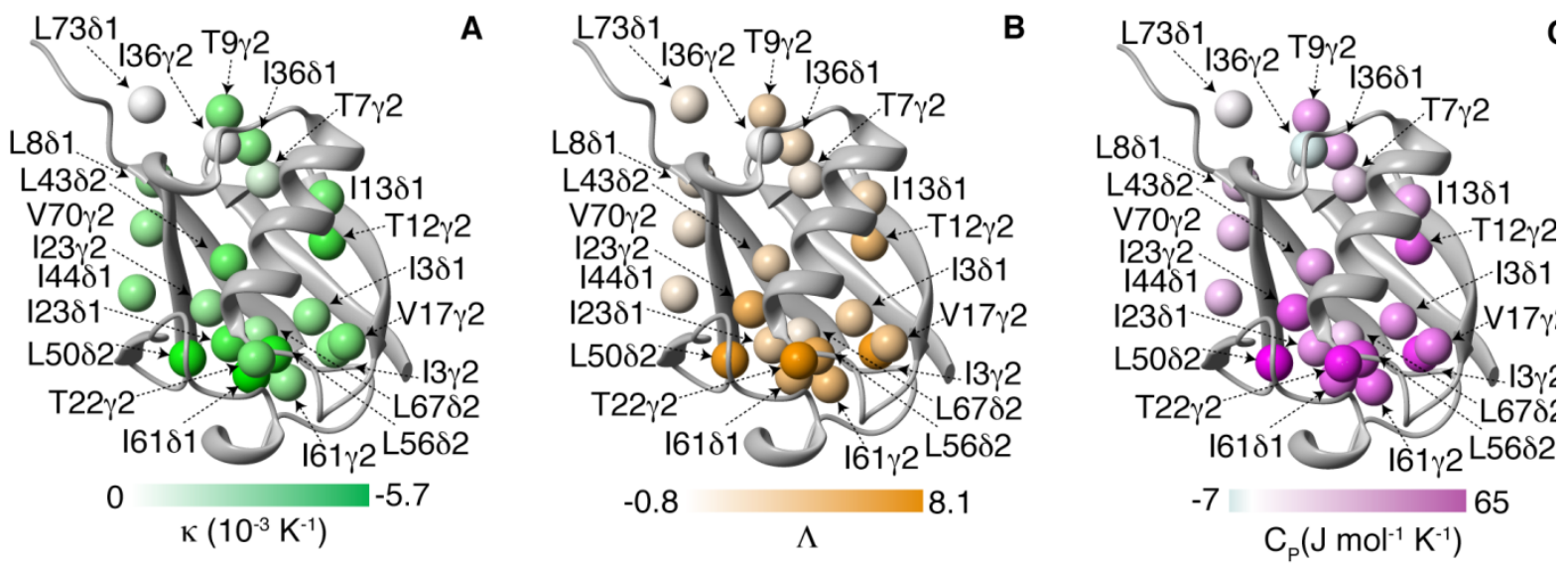

Figure 5. Distribution of the calculated thermal coefficients for methyl groups in ubiquitin (1UBQ: Vijaykumar et al., 1987). (A) Distribution of $\kappa$. (B) Distribution of the characteristic thermal coefficient $\Lambda$, which was calculated from a linear fit of $\ln \left(1-\sqrt{S_{\text {axis }}^{2}}\right)$ versus $\ln T$ (Vugmeyster et al., 2002) (C) Distribution of the heat capacity ( $C_{p}$ ), which was extracted from a linear fit of the conformational entropy ( $S_{\text {conf }}$ ) versus $\ln T$ (Privalov and Gill, 1988), where $S_{\text {conf }}=k_{B} N_{A}\left\{\ln \pi\left[3-\sqrt{1+8 \sqrt{S_{\text {axis }}^{2}}}\right]\right\}$. (Yang and Kay, 1996 and 1997)Constants $k_{B}$ and $N_{A}$ are the Boltzmann constant and Avogadro's number, respectively. Spheres represent the methyl group carbon atom. The color gradient is used to represent the magnitude of the specified thermal parameter. The figures were built with the program MOLMOL (Koradi et al., 1996) (from Sabo et al., 2012).

\section{Insight into the thermal stability of ubiquitin from the distribution of thermal coefficients}

The localization of large valued thermal coefficients near the N-terminal region of the ubiquitin is the distinguished feature of this study. At the first glance it appears to be counterintuitive that these methyl groups are clustered within the core region of the protein. Larger than the average heat capacity values $\left(C_{p, \text { average }}=28.5 \pm 18.9\right)$ in flexible regions could be explained by tendency of these residues to populate additional states more readily and mainly with respect to increase in thermal energy. Furthermore, other studies show that the $\alpha$ - 
helix and the turn between $\beta$-strands 1 and 2 located in the $\mathrm{N}$-terminal region of ubiquitin, has resilience to temperature fluctuations (Babu et al., 2004; Cordier and Grzesiek, 2002). (Went and Jackson, 2005; Brutscher et al., 1997). The denaturation of ubiquitin in a mixture of methanol and water has not shown complete unfolding but partially structured speciece called A-state is formed, in which the $\mathrm{N}$-terminal $\beta$-sheet and central $\alpha$-helix remains native like (Cordier and Grzesiek, 1997). Study of unfolding kinetic of many ubiquitin mutants revealed a highly polarized transition state containing folding nucleus, which comprises the major $\alpha$ helix and the N-terminal $\beta$-hairpin (Went and Jackson, 2005). Taken together with the observed high heat capacity in the $\mathrm{N}$-terminal region (first and second $\beta$-sheet, excluding T7 $\gamma 2$ and L8 81 in the connecting loop) and $\alpha$-helix by our method, it can be indicated that the core structural elements has been formed earlier during the protein folding procedure. The order parameters of backbone NH has been studied in several thermo-stable proteins and results showed the $\mathrm{NH}$ vectors with larger $C_{p}$ to be located within the more rigid regions of secondary structure (Vugmeyster et al., 2002; Vinther et al., 2011; Seewald et al., 2000). Additionally, the dependence of $C_{p}$ magnitude on the change in $S_{\text {conf }}$ (see equation (7)), revealed by three independent groups,(Li et al., 1996; Yang and Kay, 1996; Vugmeyster et al., 2002) that the largest fluctuation for $S_{\text {conf }}$ is seen in groups possessing high order parameter, $S^{2}$, varies between 0.7 to 0.95 . High melting temperature of $\sim 363 \mathrm{~K}$ for ubiquitin (Wintrode et al., 1994) and other thermo-stabile proteins might be related in part to the relatively large local $C_{p}$ of the methyl groups and $\mathrm{NH}$ bonds in the folded proteins reducing the magnitude of $\Delta C_{p}$ upon unfolding.

An exceptional slightly negative $C_{p}$ for I36y2 methyl group also resulted from our calculation. Negative $C_{p}$ for $\mathrm{NH}$ bonds located in secondary structural regions has been reported in two other studies (Vinther et al., 2011; Lee et al., 2002). Negative heat capacity describes a decrease in the conformational entropy with respect to temperature. If an effect 
like a hydrophobic interaction is being enhanced at higher temperature, one would expect more ordering and less entropy by increasing the temperature. As reported by Thomas and Makhatadze (2000) there is a so- called helix-capping interaction at the end of $\alpha$-helices, which plays a central role in stability of ubiquitin. It has been shown by far-UV circular dichroism (CD) spectroscopy and DSC that this important interaction is established between side chains of I36 and I30 at the C-terminus of the ubiquitin $\alpha$-helix (Thomas and Makhatadze, 2000) Accordingly, the negative $C_{p}$ imply a decrease in the mobility and a reduction in the accessible conformational space for this methyl group. This can be explained by enhancement of hydrophobic interaction between I36 and I30 with increasing temperature. 


\section{Materials and Methods}

\section{NMR spectroscopy and processing.}

Uniformly wild type ${ }^{15} \mathrm{~N},{ }^{13} \mathrm{C}$-labeled human ubiquitin was expressed and purified as described previously in our molecular biology laboratory by Karin Giller (Handel et al., 1999). For the NMR measurements, a $3.6 \mathrm{mM}$ sample of the protein was prepared in $350 \mu \mathrm{l}$ of $50 \mathrm{mM}$ sodium phosphate buffer, $100 \mathrm{mM} \mathrm{NaCl}, 0.1 \% \mathrm{NaN}_{3}$, pH 6.8 in $10 \% \mathrm{D}_{2} \mathrm{O} / 90 \% \mathrm{H}_{2} \mathrm{O}$. A series of $2 \mathrm{D}$ constant time ${ }^{13} \mathrm{C}$, ${ }^{1} \mathrm{H}$ HSQCs without decoupling of ${ }^{1} \mathrm{H}$ during ${ }^{13} \mathrm{C}$-chemical shift evolution were acquired at 14 temperature points: 275, 278, 281, 283, 286, 288, 291, 293, 296, 298, 301, 303, 305, and 308 K. All NMR experiments were done in succession on a 700 MHz Avance-III Bruker spectrometer equipped with a triple resonance probe head. The duration of constant time and INEPT delays were set to 27.8 and $2 \mathrm{~ms}$, respectively. The spectrum was measured with 1024 and 128 complex points in the direct $\left(t_{2}\right)$ and indirect $\left(t_{1}\right)$ dimensions, respectively, with 8 scans per $t_{1}$ increment. The $t_{1, \max }$ and $t_{2, \max }$ were set to 24.3 ms and 113 ms, respectively. Frequency discrimination in the indirectly detected dimension was performed with the States-TPPI scheme (Marion et al., 1989). The duration of each measurement was 59 minutes.

All time domain data processing were done in the same manner with NMRPipe software (Delaglio et al., 1995). The data were zero-padded to $8 \mathrm{k}$ and $16 \mathrm{k}$ in $t_{1}$ and $t_{2}$, respectively. After application of a time domain solvent correction, a sine-bell window function was implemented in the direct dimension, followed by Fourier transformation of $t_{2}$. For the indirect dimension, a mirror image linear prediction algorithm was employed to increase the resolution in $t_{1}$. Next, a Gaussian window function was utilized followed by Fourier transformation of $t_{1}$. Finally, a polynomial baseline correction in the frequency domain was employed in the direct dimension. 


\section{Methods for error determination.}

The intensities of each peak in the quartet were extracted using the program CARA

(Keller, 2004) and the dipolar-dipolar cross-correlated relaxation rates ( $\left.\sigma_{o b s}\right)$ were calculated applying to the equation(3). In order to estimate the errors in $\sigma_{o b s}$, the noise levels $(q)$ for each measurement were was taken from NMRPipe and the error $\left(\Delta \sigma_{o b s}\right)$ was propagated from the following relation,

$$
\Delta \sigma_{o b s}=\frac{q}{8 \Delta}\left(\frac{1}{I_{\alpha^{3}}}+\frac{1}{I_{\alpha^{2} \beta}}+\frac{1}{I_{\alpha \beta^{2}}}+\frac{1}{I_{\beta^{3}}}\right),
$$

where $\Delta$ is the duration of the constant time period and $I_{i}$ is the intensity of each peak in the quartet ( $i=\alpha^{3}, \alpha^{2} \beta, \alpha \beta^{2}$, or $\beta^{3}$ ). For the methyl group order parameters $\left(S_{a x i s}^{2}\right)$, the errors $\left(\Delta S_{a x i s}^{2}\right.$ ) were estimated by propagating the error from $\Delta \sigma_{o b s}$ substituting in the equation,

$$
\Delta S_{\text {axis }}^{2}=\frac{\Delta \sigma_{o b s}}{\sigma_{\text {rigid }}},
$$

where $\sigma_{\text {rigid }}$ is calculated from equation (5). Finally, the errors in the conformational entropy $\left(\Delta S_{\text {conf }}\right)$ were extracted by propagating the error from $\Delta S_{a x i s}^{2}$,

$$
\Delta S_{\text {conf }}=4 k_{B} N_{A} A\left|\frac{1}{(3-B) B}\right|,
$$

where

$$
\begin{gathered}
A=\frac{\Delta S_{a x i s}^{2}}{2 \sqrt{S_{a x i s}^{2}}}, \\
B=\sqrt{1+8 \sqrt{S_{a x i s}^{2}}},
\end{gathered}
$$


$k_{B}$ is the Boltzmann constant and $N_{\mathrm{A}}$ is Avogadro's number. For error estimation in the temperature dependency of $S_{a x i s}^{2}\left(\kappa=\frac{d S_{a x i s}^{2}}{d T}\right)$, the characteristic thermal coefficient ( $\left.\Lambda=\frac{d \ln \left(1-\sqrt{S_{a x i s}^{2}}\right)}{d \ln T}\right)$, and the heat capacity $\left(C_{p}=\frac{d S_{\text {conf }}}{d \ln T}\right)$, Monte Carlo simulations on 500 randomly generated data sets were done. 


\section{Conclusion}

In this part of thesis, the temperature dependence for the methyl group order parameters in ubiquitin was analyzed together with Dr. T.M. Sabo. The total amount of experimental time required to obtain the needed information were significantly reduced, with the requirement of only one $2 \mathrm{D}$ constant time ${ }^{13} \mathrm{C}$, ${ }^{1} \mathrm{H}$ HSQC measurement eliminating decoupling of ${ }^{1} \mathrm{H}$ during ${ }^{13} \mathrm{C}$-chemical shift evolution. Using set of experiments at different temperature, the extracted thermal coefficients provide a brief look into the location of ubiquitin thermo-stability near the N-terminus of the protein. Significant contribution of methyl groups in the global heat capacity of ubiquitin, as well as other proteins (Yang et al., 1997; Lee et al., 2002), can be described through the regulation of local conformational entropy. Considering temperature dependence of $\mathrm{NH}$ and methyl axis order parameters, local protein thermodynamics in residual resolution offers a powerful supplement to the already well-established methods for determining global thermodynamic parameters in proteins.

Here with the presented method using fast CCR rate measurements, the heat capacity for methyl groups can be calculated as an alternative to the other existing methods more accurately as many more temperature points can be sampled for the temperature dependence study. To our knowledge the heat capacity of methyl groups within ubiquitin is reported here for the first time. This way is a promising method for study of protein unfolding to investigate the thermodynamic features of denaturation in an atomic resolution. 


\section{Part II Metabolic coupling of NAA and lactate in cortical neurons ${ }^{3}$}

\section{Introduction}

\section{$N$-acetyl aspartate (NAA) in brain:}

NAA is the second most abundant metabolite in the brain after glutamate, which in neurons has been measured by MRS (magnetic resonance spectroscopy) to be above $10 \mathrm{mM}$ (Wiame et al., 2010; Moffet et al., 2007). This brain specific compound can be used as a diagnostic marker for monitoring the health and neuronal density in various neurological disorders, including Alzheimer’s disease, multiple sclerosis (MS) and AIDS (Moffet et al., 2007). Biosynthesis of NAA is catalyzed by NAT8L (designated as Shati and formerly as asp-NAT), an aspartate specific N-acetyltransferase transferring an acetyl residue from acetyl-CoA to aspartate (Wiame et al., 2010; Tahay et al., 2012).

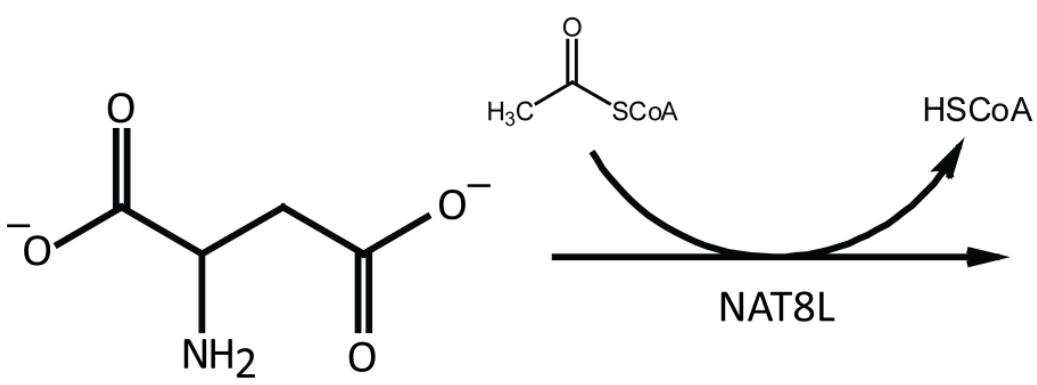

Aspartate<smiles>CC(=O)NC(CC(=O)[O-])C(=O)[O-]</smiles>

NAA

There are different reports about the subcellular localization of this membrane bound enzyme, NAT8L, namely in mitochondria (Aryannur et al., 2008), microsomes (Lu et al., 2004) and in endoplasmic reticulum (Wiame et al., 2010) . NAA is not catabolized in neurons, but it is

\footnotetext{
${ }^{3}$ In this part all stages in preparation of primary cell culture, treatments and cell imaging were done by our collaborator Dr. Kathrin Kusch in department of Neurogenetics (Prof. Klaus Armin Nave), MPI for experimental medicine. NMR sample preparation, measurements, data processing and analysis were done by the author.
} 
secreted to periaxonal space and is then taken up by oligodendrocytes, where it is hydrolyzed to aspartate and acetate by aspartoacylase (ASPA) (Moffet et al., 2007).<smiles>CC(=O)NC(CC(=O)[O-])C(=O)[O-]</smiles>

NAA<smiles>C[C@H](CO)OC(=O)C(N)CC(=O)[O-]</smiles>

Aspartate<smiles>CC(=O)O[Na]</smiles>

Acetate

It has been shown that the acetate produced by the ASPA mediated hydrolysis, is used up as a precursor for fatty acid synthesis which is required for the myelin lipids (Chakraborty et al., 2001). NAA is also the precursor for the biosynthesis of NAAG (Nacetylaspartoglutamate), the most abundant dipeptide present in the brain and is currently assumed to be a neurotransmitter (Wroblewska et al., 1998).<smiles>NC(CCC(=O)[O-])C(=O)O</smiles>

Glutamate<smiles>CC(=O)NC(CC(=O)[O-])C(=O)[O-]</smiles>

NAA<smiles>CC(=O)NC(CC(=O)[O-])C(=O)NC(CCC(=O)[O-])C(=O)[O-]</smiles>

NAAG

The other suggested function for NAA is osmoregulation and acting as a housekeeping compound by removal of large amounts of metabolic water produced after signaling activites (Baslow, 2010; Moffet et al, 2007). Up to now it has been found that NAA is not a neurotransmitter (Moffet et al., 2006), and its other proposed roles are still controversial (Wiame et al., 2010). However, in two metabolic disorders the NAA level is directly affected. Aspartoacylase (ASPA) deficiency followed by a mutation in ASPA gene causes a 
leucodystrophy known as Canavans disease. The patients with raised NAA concentration in brain and CSF (cerebrospinal fluid) are diagnosed in early infancy by dystonia, severe mental defects, blindness, megalencephalo-pathy and death on average by 18 months (Malaton et al., 2008). In the opposite case called 'hypoacetylaspartia', in which no NAA is detectable in the brain, one patient has been reported to develop truncal ataxia, developmental delay, seizure and secondary microcephaly (Wroblewska et al., 1998). This patient is homozygous for many alleles, which might be causative for some of the symptoms and additionally this disorder has been identified to be caused by a point mutation in NAT8L gene (Wiame et al., 2010).

\section{Metabolic coupling in central nervous system:}

Neurons in the central nervous system (CNS) are in interaction with glia (Fig. 6). The axons of neurons are wrapped with a myelin sheath that is provided by oligodendrocytes (Nave, 2010). The required energy for neuronal communication is reduced as a consequence of myelin evolution. The impulse propagation speed is also boosted due to the presence of the myelin sheath that makes the complex operations of the nervous systems quick and efficient (Nave, 2010; Zalc and Colman, 2000). The myelination however insulates long axons from their environment and confines their access to extracellular metabolic substrates (Nave, 2010).The Ranvier nodes are the only area exposed to the extracellular environment. If the whole metabolic demand is supported via this small area, a very efficient and special transporter have to be present at the nodes, but to date no observation supports this hypothesis.

Lee et al recently showed that monocarboxylate transporter 1 (MCT1) is the main path for lactate (or pyruvate) transport between oligodendroglia and neurons. Based on this they suggest that the oligodendroglia provide support for the neurons for maintaining axon function and neuron survival (Lee et al., 2012). It has been shown that MCT1, which is 
localized in the myelin sheath around CNS axons, can act as a transporter of lactate from oligodendroglia to axons. This suggests that MCT1-driven lactate export from oligodendroglia is the principal component for the local energy supply to axons. Gene mutation on MCT1 disrupting this transport causes axonal dysfunction and subsequent neurodegenaration (Lee et al., 2012; Rinholm and Bergersen., 2012; Pierre and Pellerin 2005).

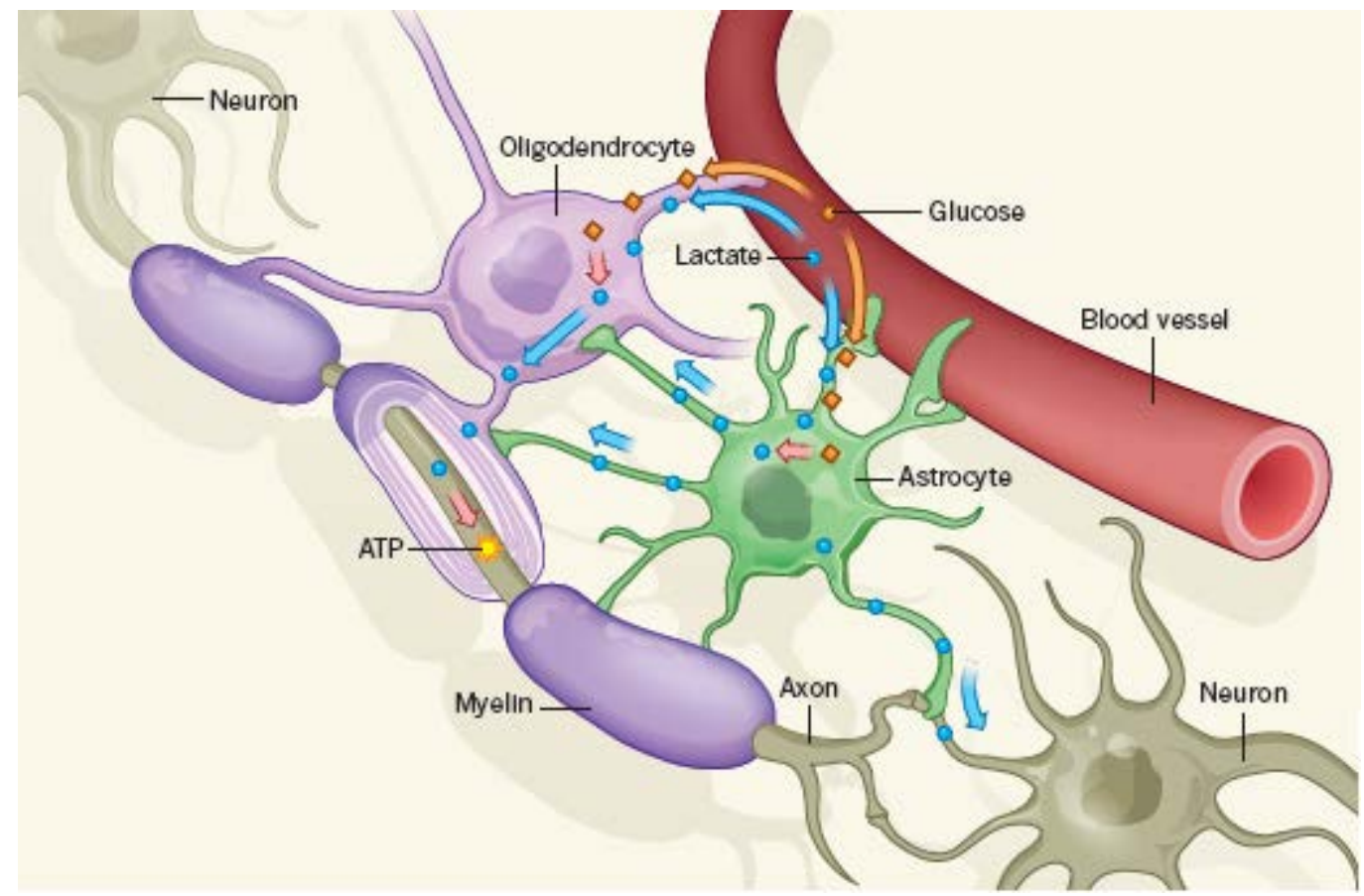

Fig 6. Metabolic coupling model between neurons (gray), astrocytes (AC) (green) and oligodendrocytes (OD) (violet). Glucose (red) is uptaken by oligodendrocytes and astrocytes, while neurons have no direct contact to blood vessels to import glucose. Glucose is converted in lactate (blue) and pyruvate (not shown here) after glycolysis in ODs and ACs. ODs secrete lactate into periaxonal space in myelin sheath and neurons uptake lactate (Fünfschilling et al., 2008; Lee et al., 2008; Rinholm and Bergersen, 2012). Astrocytes have direct contact to neurons and export lactate to neurons. This figure is adapted from Rinholm and Bergersen, (2012).

In another study by Fünfschilling et al a conditional Cox10 (protoheme IX farnesyl transferase) mutant mouse was generated, in which mature oligodendrocytes and Schwann cells failed to assemble stable mitochondrial cytochrome $c$ oxidase (COX) leading to incomplete mitochondrial respiration (Fünfschilling et al., 2012) . Interestingly, this study revealed no sign of glial cell death, demyelination and axonal degeneration or secondary inflammation in the CNS. The absence of COX does not affect post-myelinating 
oligodendrocyte survival. In vivo MRS studies of mice brains detected increased lactate concentration in mutant brains compared to controls, but just in mice under volatile anesthesia. This result indicates the rapid metabolism of aerobic glycolysis products (lactate and pyruvate) derived from oligodendrocytes within white matter tracts (Fünfschilling et al, 2012).

\section{Aim of thesis:}

The very high concentration of NAA in the brain is the main motivation point to investigate the connection of NAA to various CNS diseases and its enigmatic roll in the brain. This study is focusing on the possible metabolic and vital function of this compound based on the neuronal-oligodendroglial metabolic coupling (Fig. 6).The working hypothesis for this thesis is, that the NAA production in neurons is responsive to high concentrations of lactate to avoid lactic acidosis in energy rich situations. 


\section{Material and Methods:}

\section{Sample preparation:}

\section{Labeling:}

One of the major problems in the analysis of crowded systems like the supernatant and cell extracts is severe signal overlap. As NMR spectroscopy is sensitive only to specific isotopes such as ${ }^{1} \mathrm{H},{ }^{13} \mathrm{C},{ }^{15} \mathrm{~N}$ and ${ }^{31} \mathrm{P}$, labeling of certain compounds within a pathway may provide a potential method to avoid signal crowding by keeping other pathway products NMR silent (Lindon and Nicholson, 2008). In our study, indeed, the ${ }^{13} \mathrm{C}$ labeling could be twice advantageous. First the peaks belonging to the compounds originating from unwanted metabolic pathways are filtered out and second we can employ two-dimensional spectroscopy, which provides a correlation between ${ }^{1} \mathrm{H}$ and ${ }^{13} \mathrm{C}$ nuclei and more resolution to probe the exact metabolic products.

In this study we used the ${ }^{13} \mathrm{C}_{6}$-glucose for the labeling and detection of NAA. All metabolites originating from glucose will be ${ }^{13} \mathrm{C}$-labeled and therefore NMR visible (Fig. 7). In order to investigate the effect of other compounds such as lactate and their contribution in the NAA synthesis, the suitable labeled compound should be used, ${ }^{13} \mathrm{C}_{2}$-Lactate has been also used for labeling of NAA. 
Glc<smiles>OC[C@H]1O[C@H](O)[C@@H](O)[C@H](O)[C@H]1O</smiles>

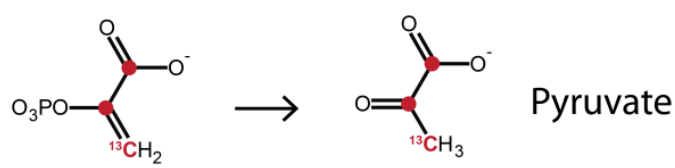

G6P<smiles>O=[Po]([O-])C[C@H]1O[C@H](O)[C@@H](O)[C@H](O)[C@H]1O</smiles><smiles>O=C(O)C(CO)C(=O)OCCO</smiles>

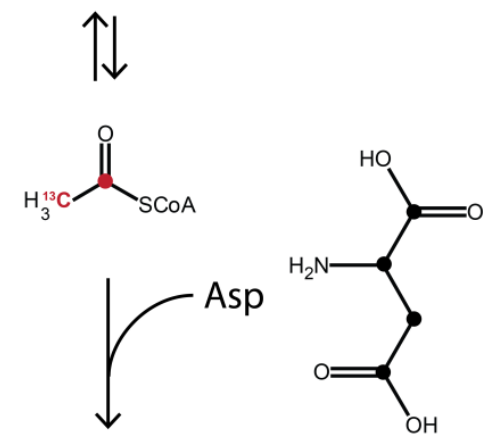

F-1,6-BP<smiles>[R6]OC[C@H]1O[C@@](O)(C[Po](=O)O)[C@H](O)[C@@H]1O</smiles>

3PG<smiles>O=C(O)C(O)COCCO</smiles><smiles>O=C(CO)CO[Na]</smiles><smiles>[C]1[C]=C1</smiles><smiles></smiles>

\section{$\rightleftarrows$}

DHAP<smiles>O=CC(O)CO</smiles><smiles>[CH]</smiles><smiles>O=C(O)C(CO)C(=O)O[Na]</smiles><smiles>CC(=O)NC(CC(=O)O)C(=O)O</smiles>

NAA

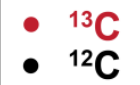

Fig 7. metabolic pathway of labeled ${ }^{13} \mathrm{C}_{6}$-glucose in the neurons up to NAA synthesis. Red dots represent the NMR visible ${ }^{13} \mathrm{C}$ and black dots indicate the NMR invisible ${ }^{12} \mathrm{C}$ atoms. Abbreviations: Glc: glucose, G6P: glucose-6-phisphate, F6P: fructose-6-phosphate, F-1,6-BP: Fructose-1,6-diphosphate, DHAP: dihydroxy acetone phosphate, GAP: glyceraldehydes phosphate, 1,3-BPG: 1,3-diphosphoglycerate, 3PG: 3-phosphoglycerate, 2PG: 2-phosphoglycerate, PEP: phosphoenol pyruvate, Asp: Aspartate.

\section{Cell culture:}

The neurons for the primary cell culture were obtained by tryptic digestion of E18 embryonic cortices of wild type (strain Wistar) rat. Cells were seeded in DMEM (Dulbecco's modified Eagle Medium) with 1\% [w/v] Glucose supplemented with 10\% [v/v] FCS (Fetal calf serum) on PLL (Poly-L-lysine) coated coverslips or cell culture dishes. Per coverslip (diameter 13mm) $2 \times 10^{5}$ cells in $0.5 \mathrm{ml}$ DMEM were applied. Per $10 \mathrm{~cm}$ cell culture dish $1.2 \times 10^{7}$ cells in $10 \mathrm{ml}$ DMEM were plated. At the consecutive day, media was exchanged to neurobasal 
medium (Neurobasal-A, Invitrogen life technologies) containing penicillin, streptomycin, B27 supplement and $25 \mathrm{mM}^{13} \mathrm{C}_{6}$ - glucose (otherwise is stated). At the days 5 and 8 in vitro half of the media was exchanged with neurobasal medium. At those days $\operatorname{AraC}^{4}(5 \mu \mathrm{M}$ and $7.5 \mu \mathrm{M}$, respectively) was also added in order to eliminate astrocytes and oligodendrocytes (Fig. 8 shows the live fixed neurons (labelled in green by anti-calbindin immunflourescence) and nuclei (labelled in blue by DAPI) of neurons, non-viable astrocytes and oligodendrocytes).

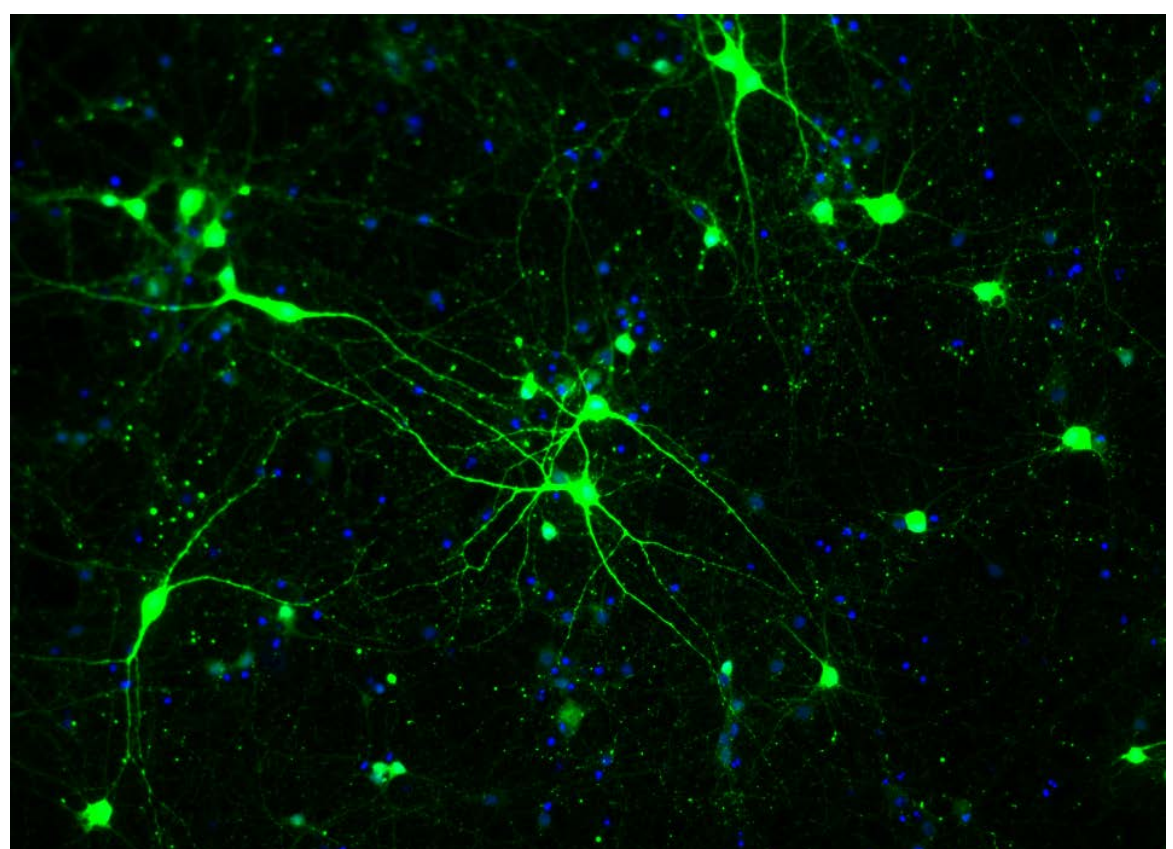

Fig 8. confocal microgram of the cell culture after treatment by the antimitotic agent AraC. The staining has been done using DAPIand anti-calbindin immunofluorescence. Living neurons are depicted by green, while dead oligodendrocytes and astrocytes were stained in blue. Picture by Dr. Kathrin Kusch (MPI-EM).

Treatment with ${ }^{13} \mathrm{C}_{2}$-L-lactate (otherwise is stated) is started at day 11 for $72 \mathrm{~h}$. Different treatments were performed in various conditions and controls, which are summarized in Table 1.

4 AraC (citarabin) is an antimitotic agent which has cytotoxic effects on proliferating cells, such as astrocytes and oligodenrocytes in our culture system, while has no toxic effects on post-mitotic cells e.g. neurons in this stage. 


\begin{tabular}{|c|c|}
\hline Sample & Condition \\
\hline 1 & $\begin{array}{l}25 \mathrm{mM}^{13} \mathrm{C}_{6}-\mathrm{Glc}^{1} \text { in Glc free NB-A } \mathrm{A}^{2} \text { Medium, No additional lac } \\
\text { or } \mathrm{NaCl}\end{array}$ \\
\hline 2 & $25 \mathrm{mM}^{13} \mathrm{C}_{6}$-Glc, $10 \mathrm{mM} \mathrm{NaCl}$ in Glc free NB-A Medium \\
\hline 3 & $\begin{array}{l}25 \mathrm{mM}^{13} \mathrm{C}_{6} \text {-Glc, } 1 \mathrm{mM} \mathrm{Na}{ }^{13} \mathrm{C}_{2}-\mathrm{L} \text {-Lac }{ }^{3} \text { and } 9 \mathrm{mM} \mathrm{NaCl} \text { in } \\
\text { Glc free NB-A Medium }\end{array}$ \\
\hline 4 & $\begin{array}{l}25 \mathrm{mM}{ }^{13} \mathrm{C}_{6} \text {-Glc, } 5 \mathrm{mM} \mathrm{Na}{ }^{13} \mathrm{C}_{2} \text {-L-Lac and } 5 \mathrm{mM} \mathrm{NaCl} \text { in Glc } \\
\text { free NB-A Medium }\end{array}$ \\
\hline 5 & $\begin{array}{l}25 \mathrm{mM}{ }^{13} \mathrm{C}_{6} \text {-Glc, } 10 \mathrm{mM} \mathrm{Na}{ }^{13} \mathrm{C}_{2} \text {-L-Lac in Glc free } N B-A \\
\text { Medium }\end{array}$ \\
\hline 6 & $25 \mathrm{mM}{ }^{13} C_{6}-$ Glc, $20 \mathrm{mM}$ Sucrose in Glc free NB-A Medium \\
\hline 7 & $30 \mathrm{mM}{ }^{13} \mathrm{C}_{6}$-Glc in Glc free NB-A Medium \\
\hline 8 & $\begin{array}{l}25 \mathrm{mM}^{13} \mathrm{C}_{6}-\mathrm{Glc}, 10 \mathrm{mM} \mathrm{Na}{ }^{12} \mathrm{C}_{2} \text {-L-Lac in Glc free } \\
\text { NB-A Medium }\end{array}$ \\
\hline 9 & $\begin{array}{l}25 \mathrm{mM}^{12} \mathrm{C}_{6} \text {-Glc, } 10 \mathrm{mM} \mathrm{Na}{ }^{13} \mathrm{C}_{2} \text {-L-Lac in Glc free NB-A } \\
\text { Medium }\end{array}$ \\
\hline
\end{tabular}

Table 3

${ }^{1}$ Glucose

${ }^{2}$ Neurobasal-A (Invitrogen life technologies, see Appendix II for details)

${ }^{3}$ L-Lactate

NMR Samples for supernatant:

Samples for analyzing cell culture the supernatants were collected at day 14 (72 hours after beginning of lactate treatment) from each well of a corresponding condition and kept frozen at $-80{ }^{\circ} \mathrm{C}$ until the measurement began. After defrosting, $5 \%$ [v/v] $\mathrm{D}_{2} \mathrm{O}$ was added to $170 \mu \mathrm{l}$ supernatant and the samples were transferred into $3 \mathrm{~mm}$ NMR tubes for measurement. 


\section{NMR Samples for cell extract:}

Cell extracts were prepared at day 14 (72 h after beginning of lactate treatment).Therefore, the cover slips with the neurons were washed twice in PBS, (phosphate buffered saline), pH 7.4, immersed in $300 \mu \mathrm{l}$ PBS, placed in a pre-heated beaker with water and irradiated for 20 sec by microwave (at 800 Watts). Boiling stops any enzymatic activity which might degrade the NAA. The crude lysate was kept frozen at $-80^{\circ} \mathrm{C}$ until measurement. After thawing, cell and glas debries were removed by centrifugation ( 2 min at $0.3 \mathrm{~g}$ ). $170 \mu \mathrm{l}$ of cell extract of neurons and were supplemented with $5 \%[\mathrm{v} / \mathrm{v}] \mathrm{D}_{2} \mathrm{O}$ and transferred to a $3 \mathrm{~mm}$ NMR tube.

NMR sample for in cell experiment:

For in cell detection of NAA cells of eight $10 \mathrm{~cm}$ plates were dissociated by scraping with a silicone cell scrapper and pooled in a $2 \mathrm{ml}$ Eppendorf tube, and were then centrifuged for 2 min at 300rpm. After separation of supernatant cells were suspended in $100 \mu \mathrm{l}$ of NB medium containing $10 \%$ [v/v] $\mathrm{D}_{2} \mathrm{O}$. The cell suspension was transferred to a $3 \mathrm{~mm}$ NMR tube for measurement. An example of how the sample appeared is presented in Fig 9.

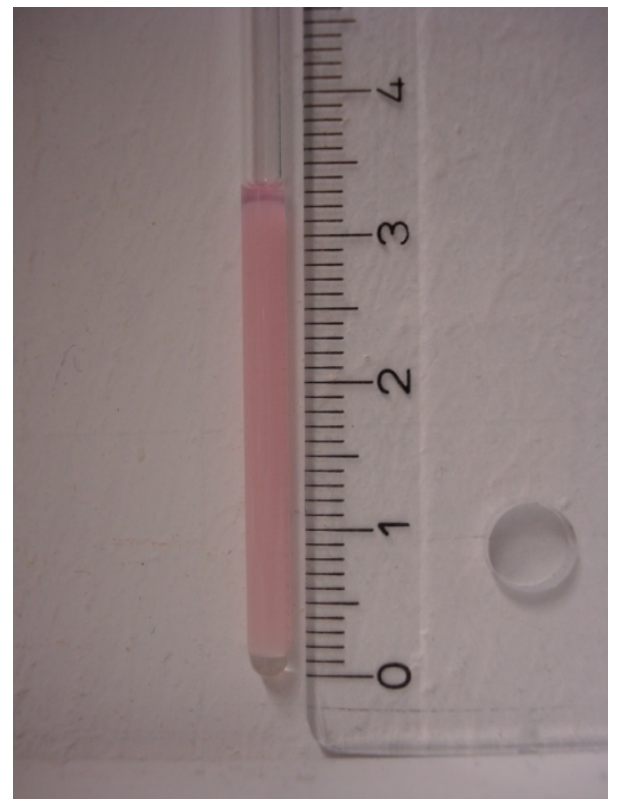

Fig 9. In cell NMR sample made of eight $10 \mathrm{~cm}$ plates in a $3 \mathrm{~mm}$ NMR tube. The cells were suspended in $100 \mu \mathrm{M}$ fresh NB medium and $10 \%$ $\mathrm{D}_{2} \mathrm{O}$. 


\section{Chemical synthesis of ${ }^{13} C_{2}-N A A$ :}

A similar standard to the generated NAA in the labeled media is needed for precise quantification. The reference compound was synthesized by Dr. Andrei Leonov (MPI-BPC) by the reaction of ${ }^{13} \mathrm{C}_{2}$-acetyl chloride and unlabeled Na L-aspartate.<smiles>[3H][13C](C)=O</smiles>

Aspartate

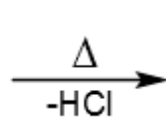<smiles>C[14CH2]NC(C)C(=O)[O-]</smiles>

NAA

Reaction for synthesis of ${ }^{13} \mathrm{C}_{2}$-labeled NAA from ${ }^{13} \mathrm{C}_{2}$-acetylchloride and aspartate

\section{NMR Spectroscopy:}

\section{$1 D$ NMR measurements:}

One dimensional proton NMR spectra were recorded on a $900 \mathrm{MHz}$ Bruker Avance spectrometer equipped with a cryogenic TCI $-{ }^{1} \mathrm{H} /{ }^{13} \mathrm{C}-{ }^{15} \mathrm{~N} /{ }^{2} \mathrm{H}$ probe head at $277 \mathrm{~K}$. Water handling is an important requirement for these measurements, since the water concentration is ca. $55 \mathrm{~mol} / \mathrm{l}$, while the concentration of the other components of the supernatant and extract samples are in $\mathrm{mmol} / \mathrm{l}$ to $\mu \mathrm{mol} / \mathrm{l}$ range. To overcome the three to six order of magnitude difference in water signal, there are several methods, called water handeling, to change the relative intensity of water signal to the rest of signals (Cavanagh et al, 2007). In this study we used presaturation method for water handling, applying a long (1.2 s) low power pulse $(7 \mu \mathrm{W})$ with the water resonance frequency prior to the $90^{\circ}$ hard pulse for the detection. This long pulse saturates the magnetization of water protons and dramatically decreases the intensity of its detected signal (Cavanagh et al, 2007). 


\section{$T_{2}$ Filtering:}

Larger molecules in the sample have longer tumbling time and therefore they feature faster transverse relaxation rate (green curve in fig. 10). Faster transverse relaxation rate of a time domain signal leads to broader signal in the spectrum, which causes overlap and large uncertainty in the intensity measurements. To discriminate and replace these signals from spectra, the magnetization should be kept in the transverse plane by series of spin-echoes, called $T_{2}$-filtering, for a relatively short period. In this period the slowly tumbling molecules lose their coherence more rapidly than the small molecules. The $T_{2}$-filtering was implemented by the CPMG-z pulse sequence from Rastelli et al (2009). This experiment uses the chemical shift evolution refocusing during the wait period (shown as yellow box in Fig. 10) and CarrPurcell-Meiboom-Gill (CPMG) spin-echo trains of the kind $\left(\tau-\pi_{\phi}-\tau\right)_{n}$ as $T_{2}$-filter.

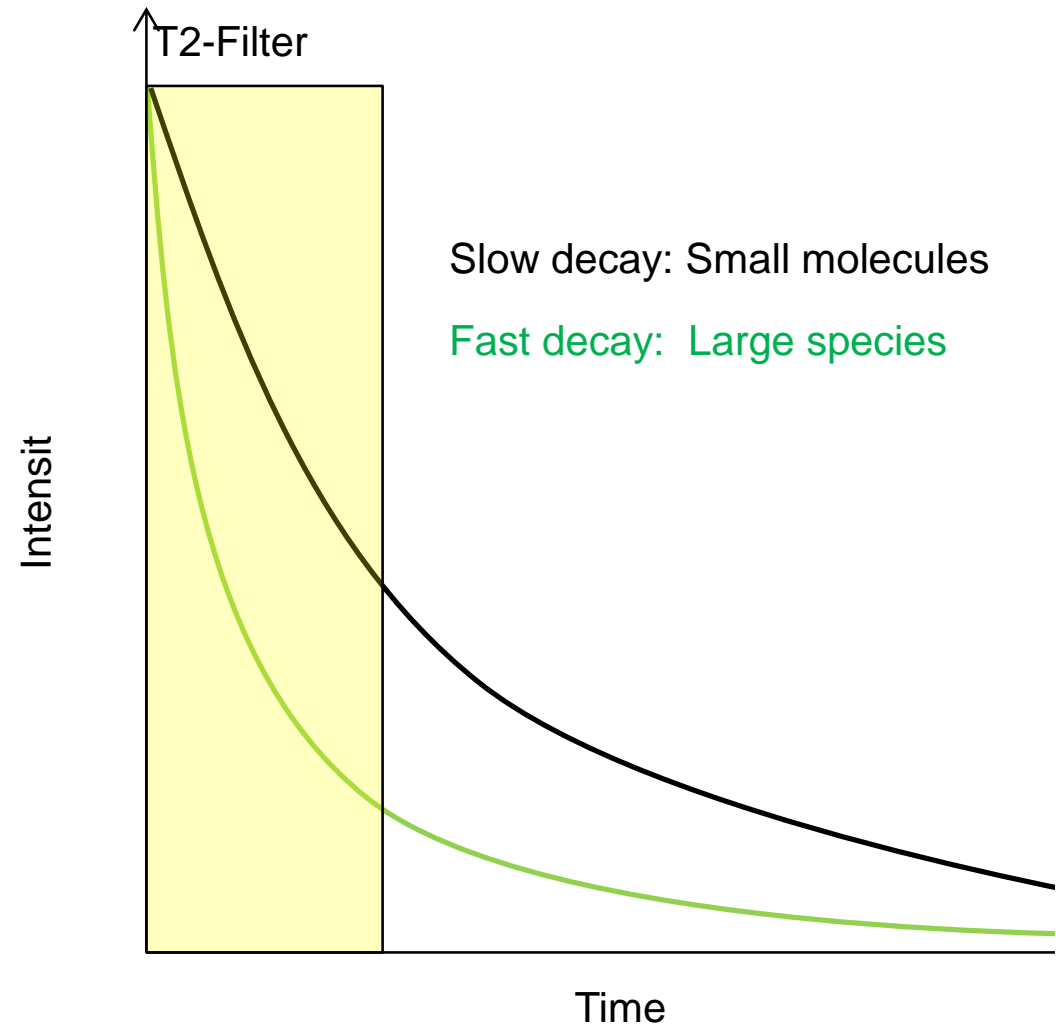

Fig $10 T_{2}$-filtering is used to eliminate the signals arising from large molecules (green) with fast transverse relaxation rate, while keeping the magnetization of small molecules (black). 
All 2D NMR measurements were done on a $700 \mathrm{MHz}$ Bruker Avance spectrometer equipped with a cryogenic TCI- ${ }^{1} \mathrm{H} /{ }^{13} \mathrm{C}-{ }^{15} \mathrm{~N} /{ }^{2} \mathrm{H}$ probe head at $277 \mathrm{~K}$. For each sample a ${ }^{13} \mathrm{C},{ }^{1} \mathrm{H}-\mathrm{HSQC}$ spectrum was recorded with ${ }^{13} \mathrm{C}$ decoupling during the acquisition. The spectra were recorded with 2048 and 300 complex points in the direct $\left(t_{2}\right)$ and the indirect $\left(t_{1}\right)$ dimensions, respectively with 32 scans per $t_{1}$ increment. Frequency discrimination was achieved with the States-TPPI scheme. Each experiment required 212 minutes.

All time domain data were processed in the same manner with TopSpin $1.2{ }^{\circledR}$ software. The data were zero filled to 4096 and 1024 point in $t_{1}$ and $t_{2}$, respectively. After implementing a time domain solvent correction, a sine-bell window function was applied and subsequently Fourier transformed in $t_{2}$ and $t_{1}$. Finally an automatic polynomial baseline correction in the frequency domain was applied in both dimensions. The position of ${ }^{13} \mathrm{C}$-methyl signal of NAA was confirmed based on its chemical shift in compare to the reference compound $\left({ }^{13} \mathrm{C}_{2}\right.$-NAA) in supernatant and cell extract. The intensity of ${ }^{13} \mathrm{C},{ }^{1} \mathrm{H}$-methyl cross peak was taken to estimate the concentration of NAA in each sample by comparison to a reference spectrum of pure ${ }^{13} \mathrm{C}_{2}$-NAA. 


\section{Results and Discussion:}

\section{Quantification of NAA using NMR}

The first and easiest attempt to quantify the NAA (without any labeling) in a solution is 1D proton NMR spectroscopy and comparison to an internal or external standard. In a 1D proton spectrum of NAA we observed four peak sets for three methyl protons (2.01 ppm), two methylene protons (2.49 and $2.67 \mathrm{ppm})$, one methine proton (4.37 ppm) and one amide proton (7.91 ppm) (Fig. 11).
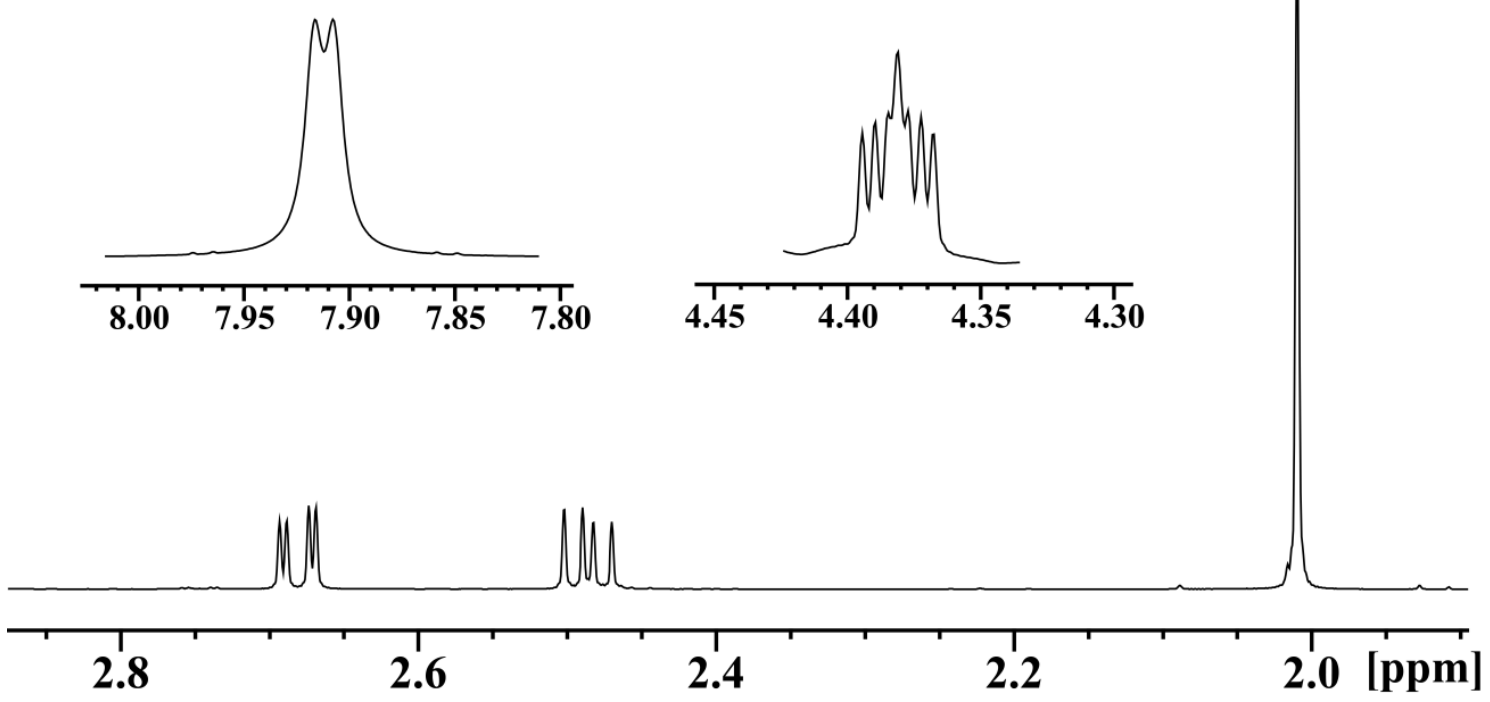

Fig 11. ${ }^{1} \mathrm{H}$-Spectrum of pure NAA $(10 \mathrm{mM})$ in $\mathrm{PBS}$ at $\mathrm{pH}=6.8$ and $298 \mathrm{~K}$ on a Bruker Avance $900 \mathrm{MHz}$ Spectrometer, equipped with a cryogenic TCI 1H/13C-15N/2H probe head. Time domain contains 16384 complex data points taken from 16 scans of presaturation experiment and multiplied by a cosine-bell function and zero filled with 16384 points prior to Fourier transformation. After Fourier transformation frequency domain data went on a zeroth order phase correction and first order polynomial baseline correction.Chemical shifts of protons from high field to low field: 2.01, singlet, $3 \mathrm{H}, \mathrm{CH}_{3} ; 2.48$, doublet of doublets, $1 \mathrm{H}, \mathrm{CH}(\mathrm{H}) ; 2.68$, doublet of doublets, $1 \mathrm{H}, \mathrm{CH}(\underline{\mathrm{H}})$; 4.38, multiplet, $1 \mathrm{H}, \mathrm{C} \underline{\mathrm{H}}$; 7.91, broad doublet, $1 \mathrm{H}, \mathrm{N} \underline{\mathrm{H}}$. (For clarity of multiplicity the spectrum extracts between $4.30-4.45$ and $7.80-8.00$ were scaled in a different manner.)

The quantification of NA in the sub-micromolar concentrations is possible, but the experiments must be run long enough. The methyl protons signal as it is a singlet and has the 
highest intensity. Therefore, we compared peaks belonging to this proton in order to measure the amount of NAA. However, in principle other peaks could also be used.
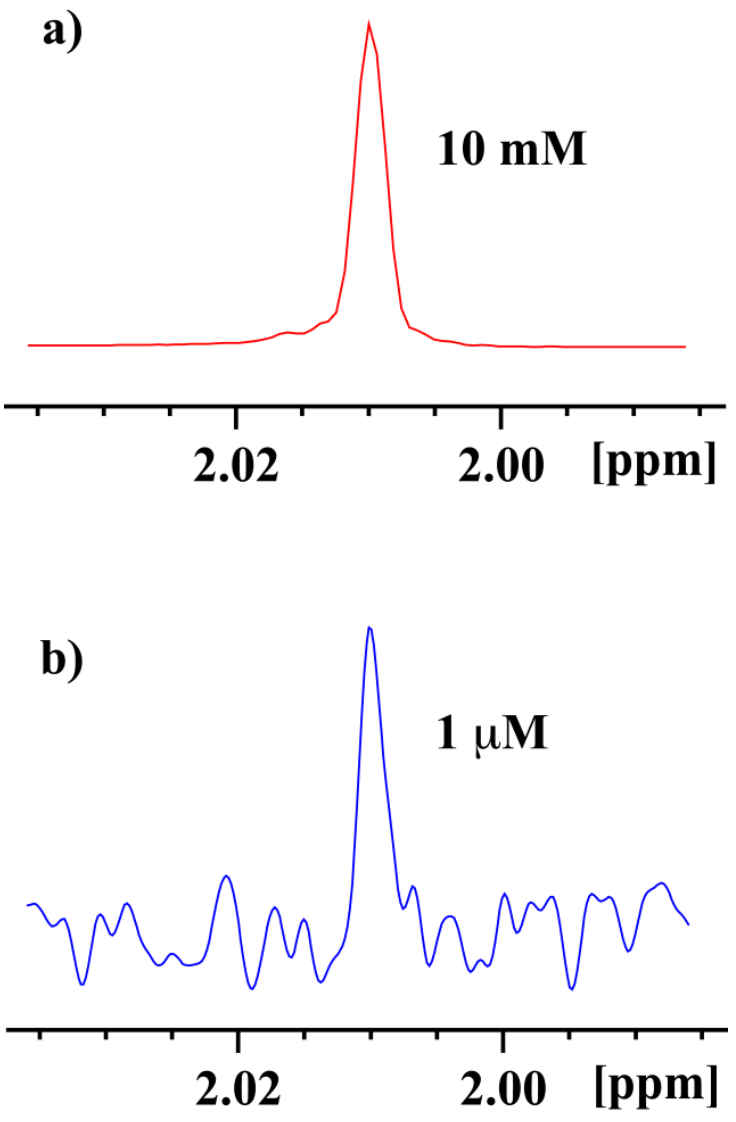

Fig 12. Comparison between ${ }^{1} \mathrm{H}-\mathrm{NMR}$ spectrum of $10 \mathrm{mM}$ NAA (red) and $1 \mu \mathrm{M}$ NAA (blue) in the methyl region. The $10 \mathrm{mM}$ spectrum is scaled down by factor of $10^{4}$. All conditions were the same in both spectra and the same as reported in Fig. 11. Else than number of scans (NS=1024).

\section{Determination of NAA in supernatant of neurons by ${ }^{1} \mathrm{H}-\mathrm{NMR}$}

The 1D proton spectrum of supernatant of neurons cultured in neurobasal medium is crowded at the chemical shift regions, where the NAA signal would arise (fig 13). Peak overlap is caused by resonance from large molecular species in the supernatant and exchangeable protons (like -OH groups). The only signal which is observed at a less crowded area is the amide proton. However, this signal is not very suitable for quantitative analysis because of its low intensity and line broadening. 


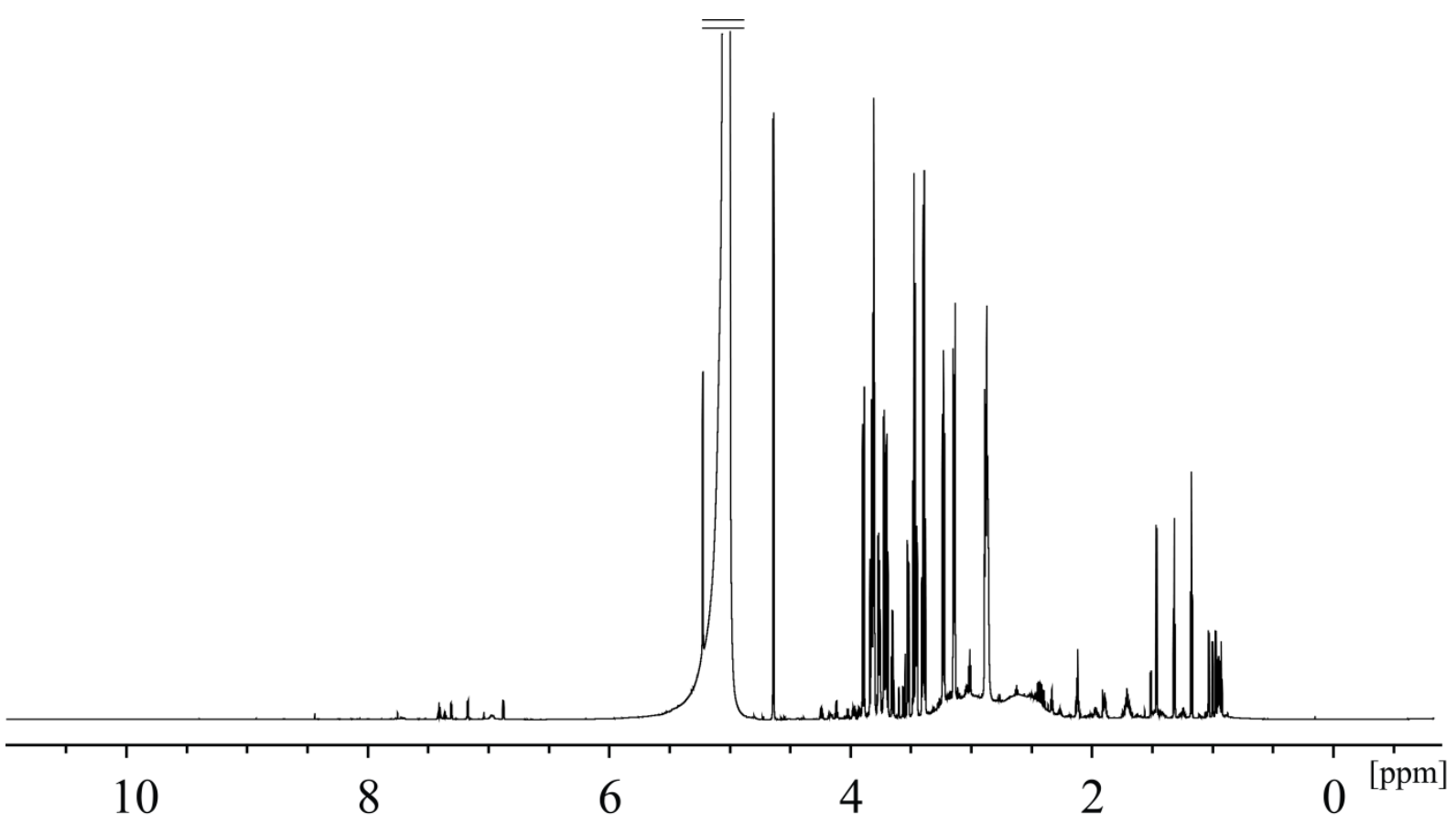

Fig 13. ${ }^{1} \mathrm{H}$-Spectrum of supernatant at $277 \mathrm{~K}$ on a Bruker Avance $900 \mathrm{MHz}$ Spectrometer, equipped with a cryogenic TCI 1H/13C-15N/2H probe head. Time domain contains 16384 complex data points taken from 16 scans of presaturation experiment and multiplied by a cosine-bell function and zero filled with 16384 points prior to Fourier transformation. After Fourier transformation frequency domain data went on a zeroth order phase correction and first order polynomial baseline correction.

In order to remove the effect of large species and exchangeable protons which have a very short transverse relaxation time we employed a $T_{2}$-Filtering method (Fig. 10 discussed in the previous chapter). The results show the exclusion of signals from large species (Fig. 13 and 14).Yet, the overlapping from the other small molecules is still hinders the separation of the desired peaks, especially the methyl signal of NAA at $2.01 \mathrm{ppm}$, which potentially could be used for quantification of NAA. The same for 1D in-cell spectra (spectrum in blue in fig. 14) it was impossible to quantify the NAA level out of methyl proton signals despite using $T_{2^{-}}$ filtering. 


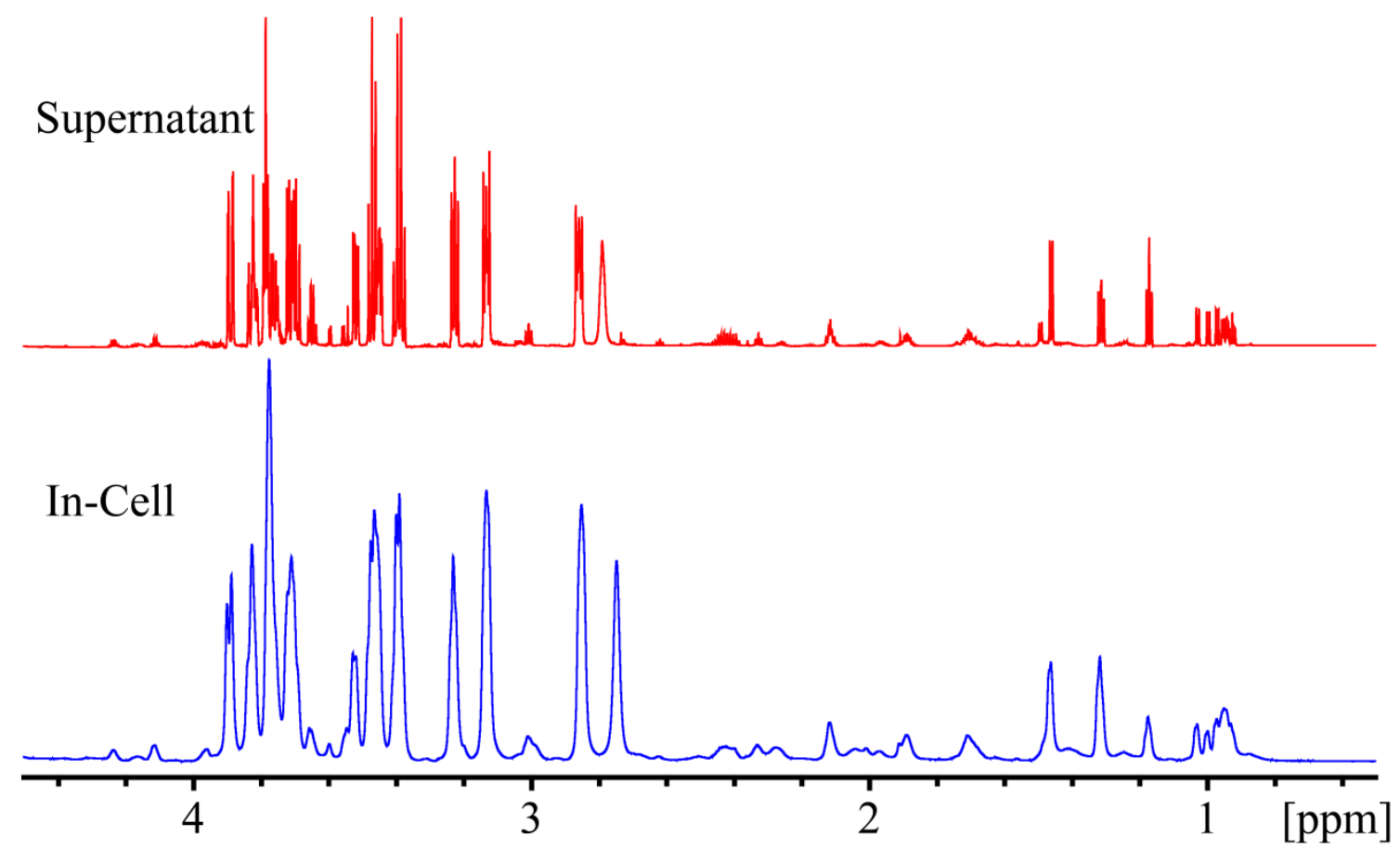

Fig 14. $\mathrm{T}_{2}$-filtered ${ }^{1} \mathrm{H}$-Spectrum of neurons (in-cell) in blue and the supernatant in red at $277 \mathrm{~K}$ on a Bruker Avance $900 \mathrm{MHz}$ Spectrometer, equipped with a cryogenic TCI 1H/13C-15N/2H probe head. Time domain contains 16384 complex data points taken from 16 scans of CMPG-z experiment with 4 loops of $10 \mathrm{~ms}$ and multiplied by a cosine-bell function and zero filled with 16384 points prior to Fourier transformation. After Fourier transformation frequency domain data went on a zeroth order phase correction and first order polynomial baseline correction.

\section{Quantification of NAA via labeling and $2 D^{13} \mathrm{C},{ }^{1} \mathrm{H}-\mathrm{HSQC}$}

As described in previous chapter the source of acetyl group of NAA is from the glycolysis pathway and its products. The $2 \mathrm{D}{ }^{13} \mathrm{C},{ }^{1} \mathrm{H}-\mathrm{HSQC}$ spectrum of the supernatant of neurons cultured in ${ }^{13} \mathrm{C}_{6}$-Glc labeled neurobasal medium shows an isolated cross peak for methyl group of NAA at $2.01 \mathrm{ppm}$ in proton and $21.94 \mathrm{ppm}$ in carbon dimension (Fig. 15a). This signal is absent, if the medium contains unlabeled glucose, revealing that glucose is the original source for the NAA acetyl group. The intensity of NAA-methyl cross peak was used for determination of NAA concentration by comparison to an external standard of NAA in NB medium at the same $\mathrm{pH}$ and temperature. In figure 15b you see 1D slices of supernatant spectrum and the external NAA standard with the scaling factor, which was used to determine the unknown concentration. 
a)

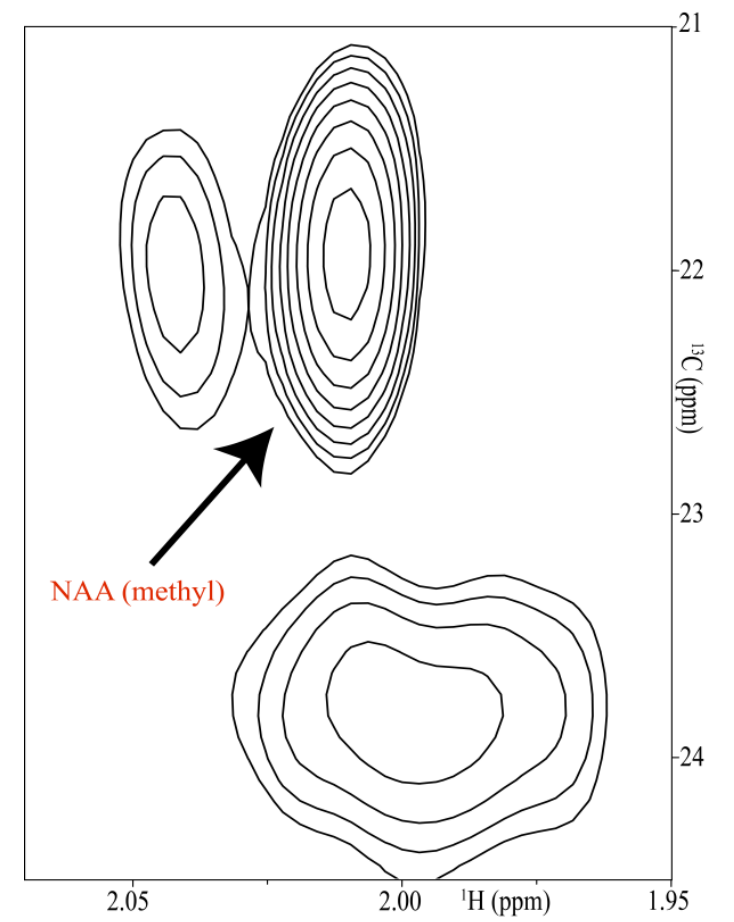

b)

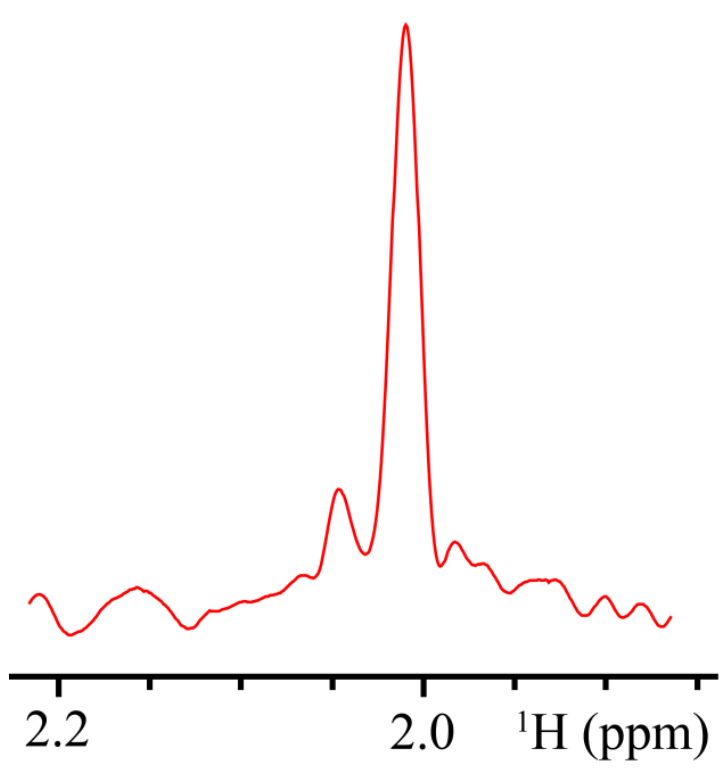

Fig 15. a. Extract of ${ }^{1} \mathrm{H},{ }^{13} \mathrm{C}-\mathrm{HSQC}$ spectrum of supernatant in the NAA-methyl region (left panel), which measured at 277 $\mathrm{K}$ on a Bruker Avance $700 \mathrm{MHz}$ Spectrometer, equipped with a cryogenic TCI $1 \mathrm{H} / 13 \mathrm{C}-15 \mathrm{~N} / 2 \mathrm{H}$ probe head. ${ }^{13} \mathrm{C},{ }^{1} \mathrm{H}-\mathrm{HSQC}$ spectrum was recorded with ${ }^{13} \mathrm{C}$ decoupling during the acquisition. The spectra were recorded with 2048 and 300 complex points in the direct $\left(t_{2}\right)$ and the indirect $\left(t_{1}\right)$ dimensions, respectively with 32 scans per $t_{1}$ increment. Frequency discrimination was achieved with the States-TPPI scheme. Each experiment required 212 minutes. The time domain data were zero filled to 4096 and 1024 point in $t_{1}$ and $t_{2}$, respectively. After implementing a time domain solvent correction, a sine-bell window function was applied and subsequently and Fourier transformed in $t_{2}$ and $t_{1}$. Finally a polynomial baseline correction in the frequency domain was applied in both dimensions. b. Right panel shows a $1 \mathrm{D}$ slice in the ${ }^{1} \mathrm{H}$ dimension for ${ }^{13} \mathrm{C}$-chemical shift at $22 \mathrm{ppm}$.

\section{$2 D$ in cell spectrum of neurons and cell extracts}

In the $2 \mathrm{D}{ }^{13} \mathrm{C},{ }^{1} \mathrm{H}-\mathrm{HSQC}$ spectrum of neurons harvested from 8 plates (corresponding to 9.6x $10^{7}$ plated cells) we observe larger line broadening than in solution as expected due to increased viscosity within the cells and subsequently slower tumbling of molecules and the field inhomogeneity within the sample. This leads to a severe overlapping in the region, where NAA signal is expected to occur (fig 16a). According to the literature

(Edden et al., 2007; Eldon et al., 2008) the other two peaks which were present at this area belong to methyl groups of NAAG and NAGA (table 4). This problem was also identified by MRS studies that attempted to quantify the amount of NAA in the brain (Edden et al., 2007). 


\begin{tabular}{lll}
\hline & ${ }^{1}$ H chemical shift (ppm) & ${ }^{13}$ C chemical shift (ppm) \\
\hline NAA-methyl & 2.01 & 21.94 \\
NAAG-methyl & 2.04 & 21.88 \\
NAGA-methyl & 2.07 & 22.13 \\
\hline
\end{tabular}

Table 4. Chemical shifts of other methyl groups around the NAA methyl peak.. All chemical shifts arereferenced with respect to DSS at $277 \mathrm{~K}$.

Using cell extracts helps us to overcome the overlapping issue as shown in figure 16b compared to the in cell spectrum (fig 16c). Estimation of NAA content was performed in the same manner as for the supernatant via an external standard.
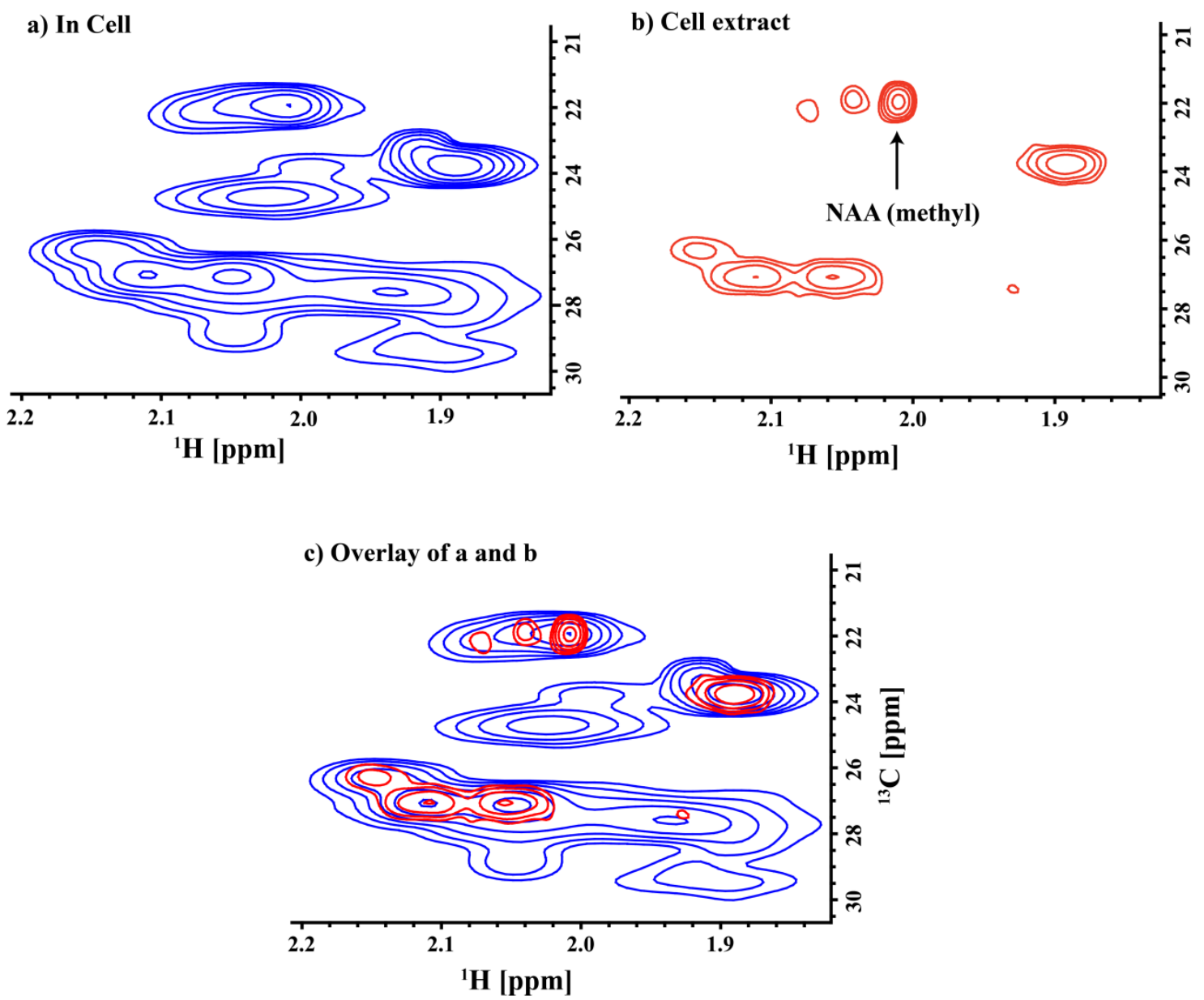

Fig 16. Extract of ${ }^{1} \mathrm{H},{ }^{13} \mathrm{C}-\mathrm{HSQC}$ spectrum of neurons (a) and cell extract (b) and overlay (c) in the NAA-methyl region, which measured at $277 \mathrm{~K}$ on a Bruker Avance $900 \mathrm{MHz}$ Spectrometer. Other experimental parameters were set as the same in the figure 10 . 


\section{Neurons respond to different lactate treatments}

The neurons were treated with ${ }^{13} \mathrm{C}_{2}$-L-lactate with increasing concentrations of $0,1,5$ and $10 \mathrm{mM}$ to investigate the main hypothesis of lactic acidosis prevention by NAA production in response to high lactate concentration. Each concentration measurement was repeated in biological triplicate. In order to keep the osmotic pressure and ionic strength constant, all samples contained 10, 9, 5 and $0 \mathrm{mM} \mathrm{NaCl}$, respectively. For the analysis of data from quantification of NAA in the samples we assume the number of cells was similar between samples. Here we report for each treatment an average of concentrations and one standard deviation as the uncertainty. The mean concentrations of NAA in supernatant and cell extract are visualized in figure 17a and 17b. A rise in NAA concentration is observed linked with increasing amount of lactate treatment. In absence of lactate a basic level of released NAA in the supernatant can be observed ([NAA $\left.]^{0 \mathrm{mM}}=1.2 \pm 0.7 \mu \mathrm{M}\right)$. The low level of NAA in the "no lactate” experiment causes a low signal to noise ratio, which results in high uncertainty of 0.7 $\mu \mathrm{M}$. As the medium is labeled with ${ }^{13} \mathrm{C}$-glucose we can conclude that the base level of NAA is synthesized from glycolysis pathway. The results of treatment with $1 \mathrm{mM}$ lactate show a slight but significant elevation of released NAA in comparison with non treated neurons $\left([\mathrm{NAA}]^{1 \mathrm{mM}}=2.4 \pm 0.2 \mu \mathrm{M} ; \mathrm{p}_{\mathrm{t} \text {-test }}=0.013<0.05\right)$. Although the values for $5 \mathrm{mM}$ lactate treatments indicate no significant change to $1 \mathrm{mM}$ treatment $\left([\mathrm{NAA}]^{5 \mathrm{mM}}=2.6 \pm 0.2 \mu \mathrm{M}\right.$; $\left.\mathrm{p}_{\text {t-test }}=0.12>0.05\right)$, it is still significantly higher than the samples without lactate treatment ( $\mathrm{p}_{\mathrm{t} \text {-test }}=0.017<0.05$ ). The neurons treated by $10 \mathrm{mM}$ of lactate released $3.9 \pm 0.3 \mu \mathrm{M}$, which is larger than NAA concentration in absence of lactate $\left(\mathrm{p}_{\mathrm{t} \text {-test }}=0.004<0.05\right)$.

In contrast with the results from the supernatant, the NAA concentration in the cell extracts stays constant at all different conditions. In absence of lactate the amount of NAA was 
respectively. No statistical significance can be reported for either of treatments ( $\left.\mathrm{p}_{\mathrm{t} \text {-test }}>0.05\right)$.
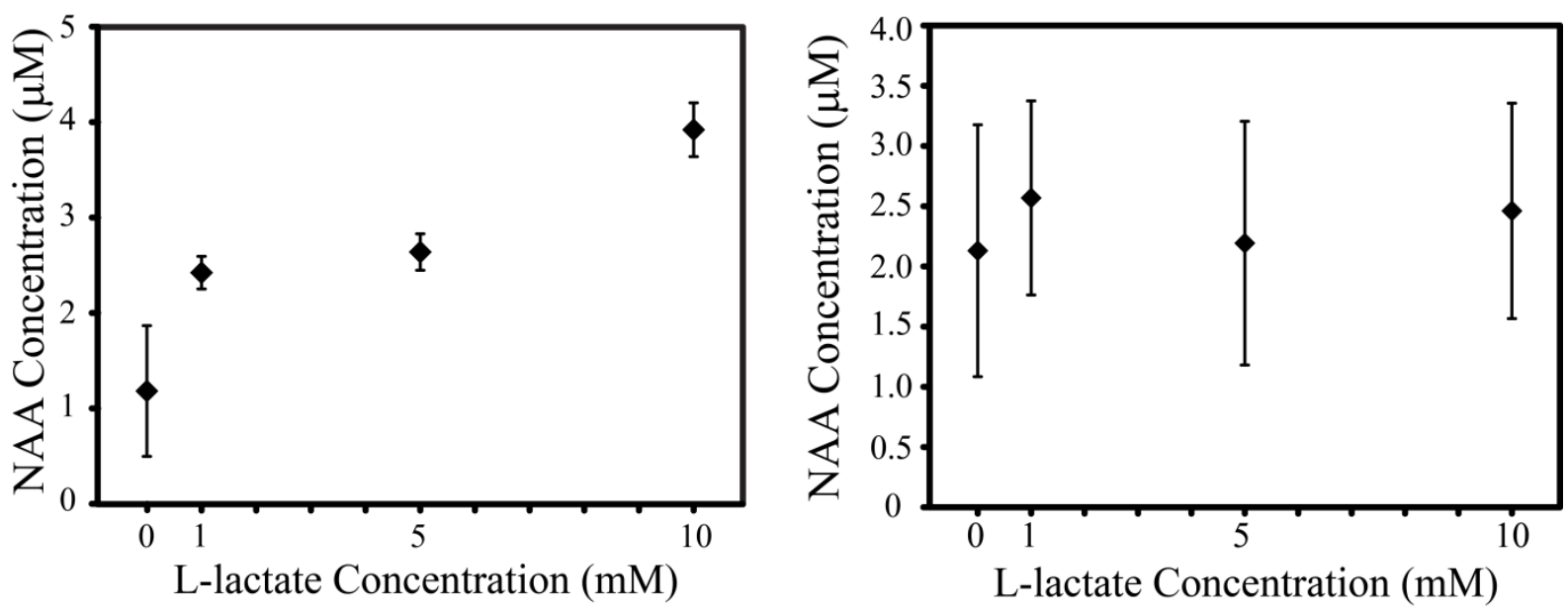

Fig 17. Effect of L-lactate treatment with different concentrations (in $0,1,5$ and $10 \mathrm{mM}$ ) on released NAA in the supernatant (left panel) as well as in the cell extract (right panel). NAA concentrations are reported for $\mathrm{N}=3$ with standard deviations as error bars ([NAA] $\pm \mathrm{SD})$

\section{Control experiments}

Addition of up to $10 \mathrm{mM}$ sodium lactate might cause changes in the culture medium based on its physicochemical properties. These variations in the environment such as osmotic pressure (Yu and Miller, 1995), ionic strength (high $\mathrm{Na}^{+}$content) (Kandel et al, 2000) or additional source of energy (Nelson and Cox, 2001), can also result in false positive observations, Here we investigate different factors to be controlled in this experiment: (i) the influence of increased osmotic pressure in the medium, (ii) the presence of $\mathrm{Na}^{+}$ions potentially interfering with neuronal membrane potentials and (iii) elevated energy equivalent availability.

First, each Na L-lactate molecule contains two particles which introduce an additional osmotic pressure to the medium. To control this situation we tried to compare $10 \mathrm{mM}$ sodium L-lactate treatment and $20 \mathrm{mM}$ sucrose treatment to an untreated culture. $20 \mathrm{mM}$ sucrose creates the same osmotic pressure as sodium L-lactate while not changing the ionic strength of the system. The result as depicted in figure 18a explains that the raise in osmotic pressure does not contribute to release of NAA from neurons into the surrounding medium. 
Second: addition of $10 \mathrm{mM}$ sodium L-lactate increases the ionic strength of the culture environment, esp. by addition of $10 \mathrm{mM} \mathrm{Na}^{+}$. This could potentially act on neuronal membrane potential, resulting in hyper polarization. In order to exclude $\mathrm{Na}^{+}$mediated effects on NAA release, we performed a control experiment in which the neurons were treated with $10 \mathrm{mM} \mathrm{NaCl}$. The detected NAA contents in the $\mathrm{NaCl}$ sample do not vary when compared with the untreated control sample (Fig. 18b). This observation indicates that the higher ionic strength is not associated with NAA release into the supernatant.
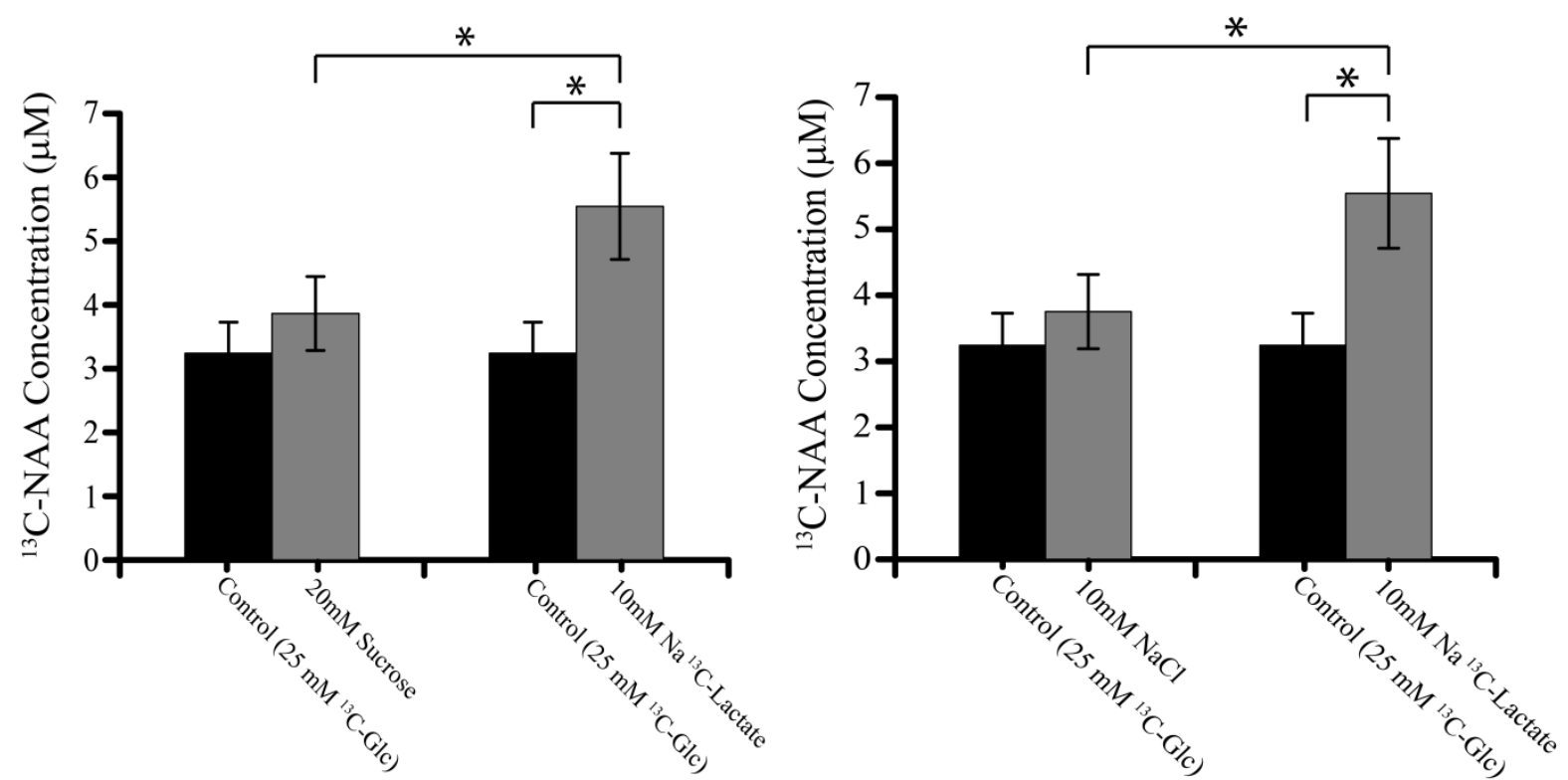

Fig 18. Control experiments for osmotic pressure (left panel) and ionic strength (right panel) of $10 \mathrm{mM}$ L-lactate treatment.

NAA concentrations are reported for $\mathrm{N}=2$ with standard deviations as error bars ([NAA] $\pm \mathrm{SD} ; *$ : $\mathrm{p}<0.05$ ).

Third, L-lactate can be used by cells for catabolism via the tricarboxylic acid cycle and subsequent oxidative phosphorylation. Considering the cellular energetic and metabolism of glucose and lactate (Nelson and Cox, 2000) the number of ATPs produced from glucose is approximately twice of ATP molecules generated from lactate. In order to check the possibility that an increased energy load of the cells results in elevated NAA secretion, an additional equivalent of glucose was added to the cultures (30 $\mathrm{mM}{ }^{13} \mathrm{C}_{6}$-glusoce instead of $25 \mathrm{mM}^{13} \mathrm{C}_{6}$-glucose). NAA synthesis or release was not increased as we treated the neurons with $30 \mathrm{mM}^{13} \mathrm{C}_{6}$-glusoce instead of $25 \mathrm{mM}^{13} \mathrm{C}_{6}$-glucose in the control (Fig 19). 


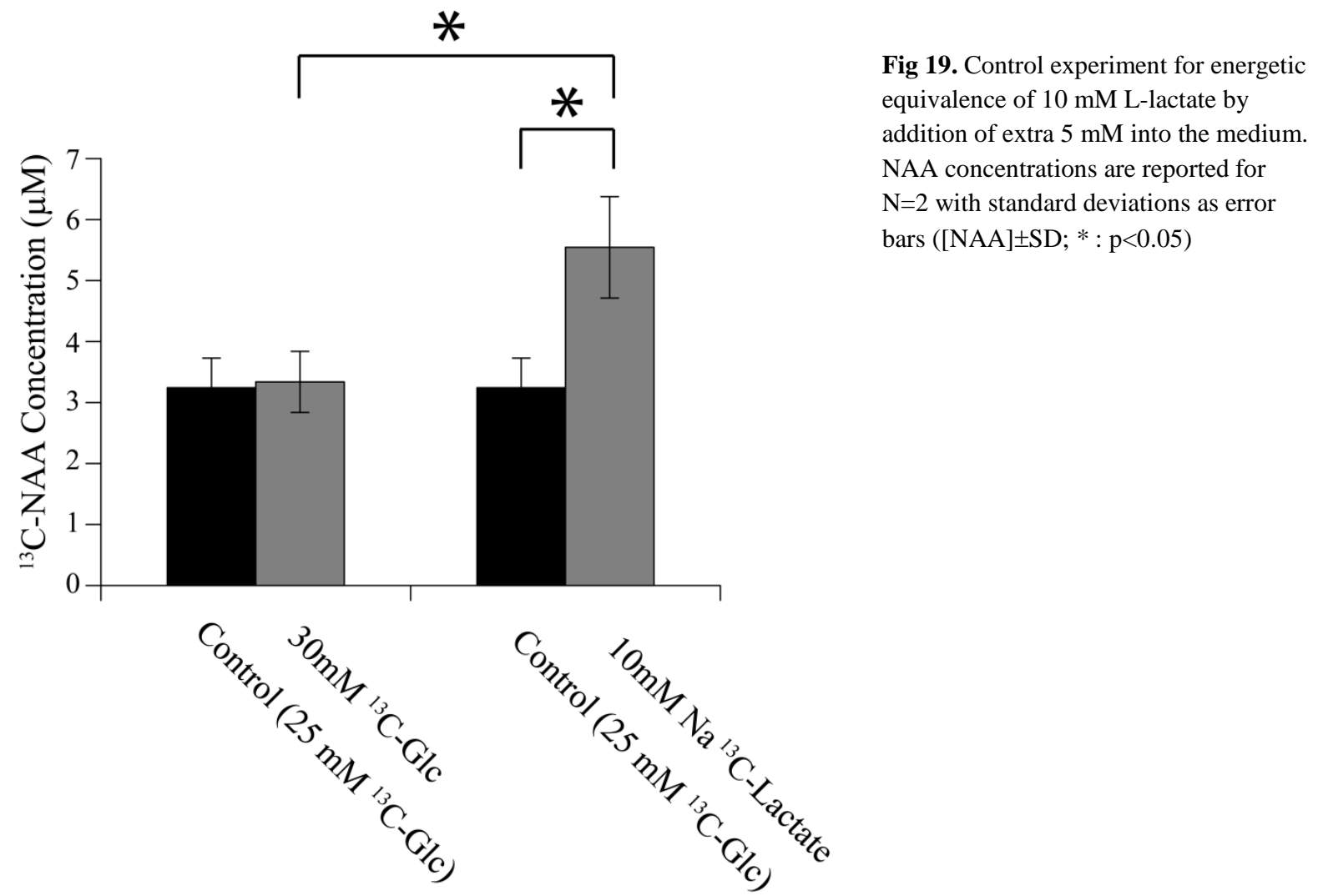

To exclude any other direct or indirect influence of Na L-lactate we employed the inverse labeling scheme, i.e. application of $25 \mathrm{mM}^{13} \mathrm{C}_{6}$-glucose and $10 \mathrm{mM}{ }^{12} \mathrm{C}$-L-lactate in one experiment while using $25 \mathrm{mM}^{12} \mathrm{C}_{6}$-glucose and $10 \mathrm{mM}^{13} \mathrm{C}_{2}$-L-lactate in the second experiment. In case of any influence of L-lactate on neurons (other than being the source for NAA synthesis) we expect an increase in NAA level in the first experiment and no NAA detection in the second one. In presence of $10 \mathrm{mM}^{13} \mathrm{C}_{2}$-lactate neurons released $3.07 \pm 0.59 \mu \mathrm{M}^{13} \mathrm{C}_{2}$-NAA and by treatment of $10 \mathrm{mM}{ }^{12} \mathrm{C}_{2}$-lactate they only produced $1.22 \pm 0.18 \mu \mathrm{M}{ }^{13} \mathrm{C}_{2}$-NAA. The summation of both experiments is in agreement with the cells cultured in $25 \mathrm{mM}{ }^{13} \mathrm{C}_{6}$-glucose and $10 \mathrm{mM}{ }^{13} \mathrm{C}$-L-lactate, namely $3.92 \pm 0.39 \mu \mathrm{M}$. The results, however, as illustrated in figure 20 deny any direct or indirect effects of Na L-lactate on neurons which might lead to NAA release in the medium. 


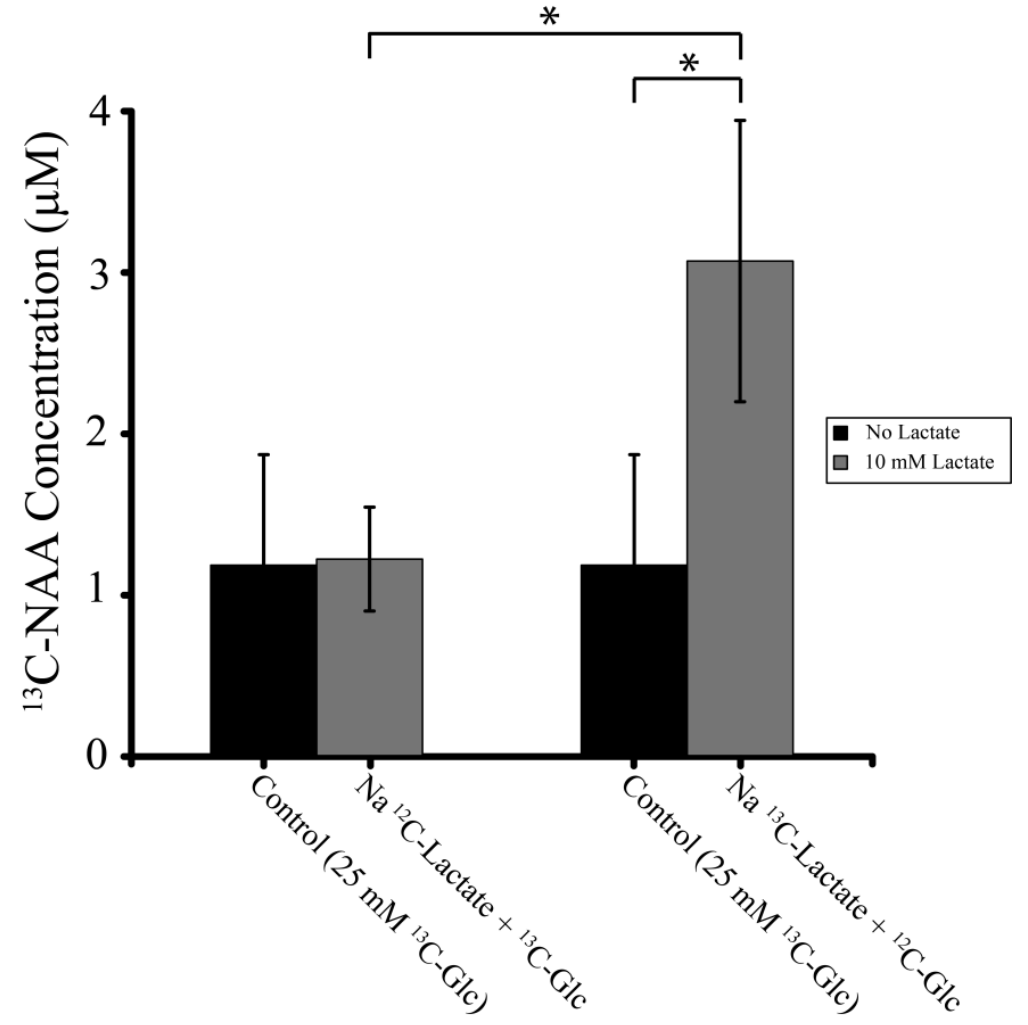

Fig 20. Inverse labeling using non labeled glucose in presence of ${ }^{13} \mathrm{C}$ labeled L-lactate as well as ${ }^{13} \mathrm{C}$-labeled glucose in presence of non labeled Llactate. NAA concentrations are reported for $\mathrm{N}=3$ with standard deviations as error bars ([NAA] $\pm S D ; *$ : $\mathrm{p}<0.05$ ) 


\section{Conclusion:}

In this work we developed a method to directly detect and quantify the amount of NAA within the supernatant of rat cortical neuron cultures, which is based on a specific isotope labeling scheme and two dimensional NMR spectroscopy. As hypothesized the NAA production in the neurons is dependent on lactate being present. The experiments presented here showed an increasing level of secreted NAA in the supernatant as a function of elevated lactate concentrations in the culture medium. Different control experiments revealed that the induced osmotic pressure, ionic strength of sodium lactate or increased energy supply were not the cause to promote NAA synthesis from glucose or the release of it. The internally produced lactate as one of the products of the glycolysis pathway was not evident as the only source for the NAA synthesis. The alternative hypothesis that assumes a stimulatory effect of lactate molecules on neurons to induce the release of NAA without being involved in the NAA synthesis, were not supported by the results using treatment of unlabeled lactate on neurons. The intracellular concentration of NAA was estimated to be rather constant at different lactate treatments. This might show that neurons are trying to keep the intracellular concentration of NAA at the certain level, while the excess amount is released to the supernatant.

According to the work of Fünfschilling et al., oligodendrocytes remain intact in the absence of complete glucose oxidation (like Warburg effect) (Fünfschilling et al., 2012) and stay functional by means of glycolysis pathway. The products of this pathway such as pyruvate and lactate are released into the periaxonal space, where neurons take up these compounds (Lee et al., 2012). Putting together that neurons posses a transporter for lactate, namely MCT2 to import it from extracellular medium (Lee et al., 2012) and proof of a neurospecific enzyme which transforms L-aspartate to NAA using Acetyl-CoA, called NAT8L as observed in knockout mice (unpublished results) we can suggest that the synthesis of NAA is the primary pathway to buffer the excess lactate to avoid acidosis at resting conditions. 


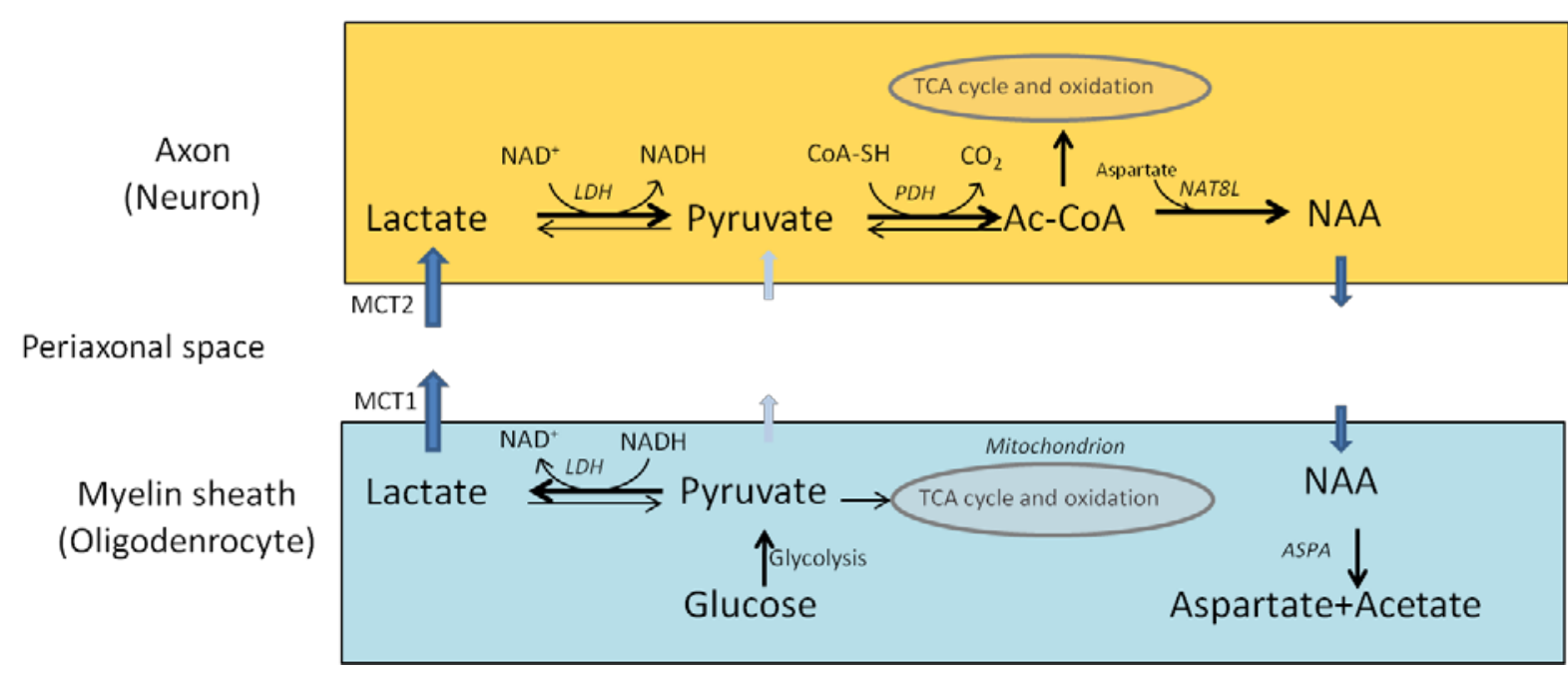

Fig 21. Metabolic pathway model for NAA synthesis and release in the myelinated axons. Although all the steps are well known, but the key function of NAA is still unclear, which was the main goal of this thesis.

The finding of this study is shedding light on the unknown function of NAA in the brain by interconnecting the role of lactate in the myelinated neurons to the correlation of NAA synthesis and present amount of lactate.For further investigation of NAA role as an well feeding signal from neurons, treatment of oligondendoglia with different NAA concentration is suggested to observe the lactate release profile of oligodendrocytes. 


\section{References:}

Abragam, A. (1961) “Principles of nuclear magnetism”. pp. 1-599. Clarendon Press, Oxford.

Akke, M., Bruschweiler, R.,Palmer, A.G. (1993) Nmr Order Parameters and Free-Energy an Analytical Approach and Its Application to Cooperative Ca2+ Binding by Calbindin-D(9k). J Am Chem Soc 115: 9832-9833.

Ariyannur, P. S., Madhavarao, C. N. and Namboodiri, A. M. (2008) N-acetylaspartate synthesis in the brain: mitochondria vs. microsomes. Brain Res. 1227: 34-41.

Baldwin, R.L. (1986) Temperature-Dependence of the Hydrophobic Interaction in Protein Folding. Proc Natl Acad Sci USA 83: 8069-8072.

Brainard, J.R. and Szabo, A. (1981) Theory for nuclear magnetic relaxation of probes in anisotropic systems: application to cholesterol in phospholipid vesicles. Biochemistry 20, 4618-4628.

Brutscher, B., Bruschweiler, R. and Ernst, R.R. (1997) Backbone dynamics and structural characterization of the partially folded A state of ubiquitin by H-1, C-13, and N-15 nuclear magnetic resonance spectroscopy. Biochemistry 36: 13043-13053.

Cavanagh, J., Fairbrother, W.J., Palmer III, A.G., Rance, M. and Skelton, N.J. (2007) “Protein NMR Spectroscopy”. pp. 1-885. Academic Press, Elsevier Inc.

Chakraborty, G., Mekala, P., Yahya, D., Wu, G. and Ledeen, R. W. (2001) Intraneuronal Nacetylaspartate supplies acetyl groups for myelin lipid synthesis: evidence for myelinassociated aspartoacylase. J. Neurochem. 78, 736-45.

Cordier, F. and Grzesiek, S. (2002) Temperature-dependence properties as studied by of protein hydrogen bond high-resolution NMR. J Mol Biol 317: 739-752. 
Delaglio, F, Grzesiek, S, Vuister, GW, Zhu, G, Pfeifer, J,Bax, A (1995) Nmrpipe - a Multidimensional Spectral Processing System Based on Unix Pipes. J Biomol NMR 6: 277-293.

Edden, R.A.E., Pomper, M.G., Barker, P.B. (2007) In vivo differentiation of N-acetyl aspartyl glutamate from N-acetyl aspartate at 3-Tesla. Mag. Res. in Med. 57, 977982.

Eldon L. Ulrich; Hideo Akutsu; Jurgen F. Doreleijers; Yoko Harano; Yannis E. Ioannidis; Jundong Lin; Miron Livny; Steve Mading; Dimitri Maziuk; Zachary Miller; Eiichi Nakatani; Christopher F. Schulte; David E. Tolmie; R. Kent Wenger; Hongyang Yao; John L. Markley;(2008) "BioMagResBank", Nucleic Acids Research 36, D402-D408

Fares, C., Lakomek, N.A., Walter, K.F.A., Frank, B.T.C., Meiler, J.,Becker, S., Griesinger, C. (2009) Accessing ns-mu s side chain dynamics in ubiquitin with methyl RDCs. J Biomol NMR 45: 23-44.

Fünfschilling U., Supplie LM., Mahad ,D., Boretius, S., Saab AS., Edgar ,J. , Brinkmann GB., Kassmann CM., Tzvetanova ID., Möbius, W., Diaz, F., Meijer, D., Suter, U., Hamprecht, B., Sereda, MW., Moraes, CT., Frahm, J., Goebbels, S. and Nave, K,-A. (2012) Glycolytic oligodendrocytes maintain myelin and long-term axonal integrity. Nature 485, 517-521.

Gans, P., Hamelin, O., Sounier, R., Ayala, I., Dura, M.A., Amero, C.D., Noirclerc-Savoye, M., Franzetti, B., Plevin, M.J.,Boisbouvier, J. (2010) Stereospecific isotopic labeling of methyl groups for NMR spectroscopic studies of high-molecular-weight proteins. Angew Chem Int Ed Engl 49: 1958-1962.

Godoy-Ruiz, R., Guo, C.,Tugarinov, V. (2010) Alanine methyl groups as NMR probes of molecular structure and dynamics in high-molecular-weight proteins. J Am Chem Soc 
132: $18340-18350$.

Gomez, J., Hilser, V.J., Xie, D.,Freire, E. (1995) The Heat-Capacity of Proteins. Proteins 22: 404-412.

Goto, N.K., Gardner, K.H., Mueller, G.A., Willis, R.C.,Kay, L.E. (1999) A robust and costeffective method for the production of Val, Leu, Ile (delta 1) methyl-protonated 15N-, 13C-, 2H-labeled proteins. J Biomol NMR 13: 369-374.

Handel, T.M., Johnson, E.C., Lazar, G.A., Desjarlais, J.R. (1999) Solution structure and dynamics of a designed hydrophobic core variant of ubiquitin. Struct Fold Des 7: 967-976.

Kay, LE, Bull, TE, Nicholson, LK, Griesinger, C, Schwalbe, H, Bax, A,Torchia, DA (1992) The Measurement of Heteronuclear Transverse Relaxation-Times in Ax3 Spin Systems Via Polarization-Transfer Techniques. J Magn Reson 100: 538-558.

Kay, LE, Torchia, DA,Bax, A (1989) Backbone Dynamics of Proteins as Studied by N-15 Inverse Detected Heteronuclear Nmr-Spectroscopy - Application to Staphylococcal Nuclease. Biochemistry 28: 8972-8979.

Kay, LE,Torchia, DA (1991) The Effects of Dipolar Cross-Correlation on C-13 MethylCarbon T1, T2, and Noe Measurements in Macromolecules. J Magn Reson 95: 536547.

Keller, R (2004) Computer Aided Resonance Assignment. http://caranmrch/.

Koradi, R, Billeter, M,Wuthrich, K (1996) MOLMOL: A program for display and analysis of macromolecular structures. J Mol Graphics 14: 51-55.

Lee, A.L. and Wand, A.J. (2001) Microscopic origins of entropy, heat capacity and the glass transition in proteins. Nature 411: 501-504. 
Lee, AL, Flynn, PF,Wand, AJ (1999) Comparison of H-2 and C-13 NMR relaxation techniques for the study of protein methyl group dynamics in solution. J Am Chem Soc 121: 2891-2902.

Lee, AL, Sharp, KA, Kranz, JK, Song, XJ,Wand, AJ (2002) Temperature dependence of the internal dynamics of a calmodulin-peptide complex. Biochemistry 41: 13814-13825.

Lee, D., Hilty, C., Wider, G. and Wuthrich, K. (2006) Effective rotational correlation times of proteins from NMR relaxation interference. J Magn Reson 178: 72-76.

Lee, Y., Morrison, BM., Li, Y., Lengacher, S., Farah MH., Hoffman, PN., Liu, Y., Tsingalia, A., Jin, L., Zhang, PW., Pellerin, L., Magistretti, PJ. and Rothstein, JD. (2012) Oligodendroglia metabolically support axons and contribute to neurodegeneration. Nature 487, 443-448.

Li, ZG, Raychaudhuri, S,Wand, AJ (1996) Insights into the local residual entropy of proteins provided by NMR relaxation. Protein Science 5: 2647-2650.

Liao, X, Long, D, Li, DW, Bruschweiler, R,Tugarinov, V (2012) Probing side-chain dynamics in proteins by the measurement of nine deuterium relaxation rates per methyl group. J Phys Chem B 116: 606-620.

Lipari, G,Szabo, A (1982a) Model-Free Approach to the Interpretation of Nuclear MagneticResonance Relaxation in Macromolecules .1. Theory and Range of Validity. J Am Chem Soc 104: 4546-4559.

Lipari, G,Szabo, A (1982b) Model-Free Approach to the Interpretation of Nuclear MagneticResonance Relaxation in Macromolecules .2. Analysis of Experimental Results. J Am Chem Soc 104: 4559-4570.

Liu, WD, Zheng, Y, Cistola, DP,Yang, DW (2003) Measurement of methyl C-13-H-1 cross- 
correlation in uniformly C-13-, N-15-, labeled proteins. J Biomol NMR 27: 351-364.

Lu, Z. H., Chakraborty, G., Ledeen, R. W., Yahya, D. and Wu, G. (2004) N-acetylaspartate synthase is bimodally expressed in microsomes and mitochondria of brain. Mol. Brain Res. 122: 71-78.

Malaton, R., Michals, K., Sebeta, D., Deanching, M. Gashkoff, P. and Casanova, J. (1988) Aspartoacylase deficiency and $\mathrm{N}$-acetylaspartatic aciduria in patients with Canavan disease. Am. J. Med. Genet. 29, 463-471.

Mandel, AM, Akke, M,Palmer, AG (1996) Dynamics of ribonuclease H: Temperature dependence of motions on multiple time scales. Biochemistry 35: 16009-16023.

Marion, D, Ikura, M, Tschudin, R,Bax, A (1989) Rapid Recording of 2d Nmr-Spectra without Phase Cycling - Application to the Study of Hydrogen-Exchange in Proteins. J Magn Reson 85: 393-399.

Millet, O, Muhandiram, DR, Skrynnikov, NR,Kay, LE (2002) Deuterium spin probes of sidechain dynamics in proteins. 1. Measurement of five relaxation rates per deuteron in (13)C-labeled and fractionally (2)H-enriched proteins in solution. J Am Chem Soc 124: 6439-6448.

Moffett, J. R. and Namboodiri, A. M. (2006) Preface: a brief review of N-acetylaspartate. Adv. Exp. Med. Biol. 576, vii-xiii.

Moffett, J.R., Ross, B., Arun, P., Madhavarao, C.N., Namboodiri, A.M.A. (2007) NAcetylaspartate in the CNS: From neurodiagnostics to neurobiology. Progress in Neurobiology 81: 89-131.

Muhandiram, DR, Yamazaki, T, Sykes, BD,Kay, LE (1995) Measurement of H-2 T-1 and T1p Relaxation-Times in Uniformly C-13-Labeled and Fractionally H-2-Labeled 
Proteins in Solution. J Am Chem Soc 117: 11536-11544.

Müller, N, Bodenhausen, G,Ernst, RR (1987) Relaxation-Induced Violations of Coherence Transfer Selection-Rules in Nuclear-Magnetic-Resonance. J Magn Reson 75: 297334.

Nash, L.K. (1974) “Elements of Statistical Thermodynamics”, Addison-Wesley, Reading, MA.

Nave K,-A. (2010) Myelination and the trophic support of long axons. Nat. Rev. 11, 275283.

Pierre, K. and Pellerin, L. (2005) Monocarboxylate transporters in the central nervous system: distribution, regulation and function. J. Neurochem. 94, 1-14.

Prabhu, NV,Sharp, KA (2005) Heat capacity in proteins. Annual Review of Physical Chemistry 56: 521-548.

Privalov, PL,Gill, SJ (1988) Stability of Protein-Structure and Hydrophobic Interaction. Adv Protein Chem 39: 191-234.

Rasterilli, F., Jhu, Satadru and Mancin, F. (2009) Seeing through Macromolecules: $T_{2^{-}}$ Filtered NMR for the purity assay of functionalized nanosystems and the screening of biofluids. J. Amer. Chem. Soc. 131, 14222-14224.

Rinholm, JE. and Bergersen, LH. (2012) The wrap that feeds the neurons. Nature 487, 435436.

Ruschak, AM, Velyvis, A,Kay, LE (2010) A simple strategy for (1)(3)C, (1)H labeling at the Ile-gamma2 methyl position in highly deuterated proteins. J Biomol NMR 48: 129135.

Sabo, T. M., Bakhtiari, D., Walter, K.F.A., McAllaister, R., Giller, K., Becker, S., 
Griesinger, C., Lee, D. (2012) Thermal coefficients of methyl groups within ubiquitin. Protein Science, 21: 562-570.

Schwalbe, H., Carlomagno, T., Henning, M., Junker, J., Reif, B., Richter, C. and Griesinger, C. (2001) Cross-Correlated Relaxation for Measurement of Angels between Tensorial Interactions. Methods in Enzymology 388, 35-81.

Seewald, MJ, Stone, MJ, Seewald, MJ, Pichumani, K, Stowell, C, Tibbals, BV,Regan, L. and Stone, MJ. (2000) The role of backbone conformational heat capacity in protein stability: Temperature dependent dynamics of the B1 domain of Streptococcal protein G. Protein Science 9: 1177-1193.

Tahay G., Wiame, E., Tyteca, D., Pierre Courtoy, P.J., and Van Schaftingen, E. (2012) Determinants of the enzymatic activity and the subcellular localization of aspartate Nacetyltransferase. Biochem. J. 441: 105-112.

Thomas, ST,Makhatadze, GI (2000) Contribution of the 30/36 hydrophobic contact at the Cterminus of the alpha-helix to the stability of the ubiquitin molecule. Biochemistry 39: 10275-10283.

Tugarinov, V, Hwang, PM, Ollerenshaw, JE,Kay, LE (2003) Cross-correlated relaxation enhanced 1H[bond]13C NMR spectroscopy of methyl groups in very high molecular weight proteins and protein complexes. J Am Chem Soc 125: 10420-10428.

Vijaykumar, S, Bugg, CE,Cook, WJ (1987) Structure of Ubiquitin Refined at 1.8 a Resolution. J Mol Biol 194: 531-544.

Vinther, J.M., Kristensen, SM and Led, JJ. (2011) Enhanced Stability of a Protein with Increasing Temperature. J Am Chem Soc 133: 271-278.

Vugmeyster, L, Trott, O, McKnight, CJ, Raleigh, DP,Palmer, AG (2002) Temperature- 
dependent dynamics of the villin headpiece helical subdomain, an unusually small thermostable protein. J Mol Biol 320: 841-854.

Wand, AJ, Babu, C.R.,Hilser, V.J. and Wand, A.J. (2004) Direct access to the cooperative substructure of proteins and the protein ensemble via cold denaturation. Nat Struct Mol Biol 11: 352-357.

Wand, AJ, Song, X.J., Flynn, P.F.,Sharp, K.A. and Wand, A.J. (2007) Temperature dependence of fast dynamics in proteins. Biophys J 92: L43-L45.

Went, H.M. and Jackson, S.E. (2005) Ubiquitin folds through a highly polarized transition state. Protein Eng Des Sel 18: 229-237.

Wiame, E., Tyteca, D., Pierrot, N., Collard, F., Amyere, M., Noel, G., Desmedt, J., Nassogne, M.-C., Vikkula, M., Octave, J.-N., Vincent, M.-F., Courtoy, P.J., Bolthauser, E. and Van Schaftingen, E. (2010) Molecular identification of aspartate N-acetyltransferase and its mutation in hypoacetylaspartia. Biochem. J. 425:127-136.

Wintrode, PL, Makhatadze, GI,Privalov, PL (1994) Thermodynamics of Ubiquitin Unfolding. Proteins 18: 246-253.

Wroblewska, B., Santi, M. R. and Neale, J. H. (1998) N-acetylaspartylglutamate activates cyclic AMP-coupled metabotropic glutamate receptors in cerebellar astrocytes. Glia 24, 172-179.

Yang, DW and Kay, LE (1996) Contributions to conformational entropy arising from bond vector fluctuations measured from NMR-derived order parameters: Application to protein folding. J Mol Biol 263: 369-382.

Yang, DW, Mok, YK, FormanKay, JD, Farrow, NA and Kay, LE (1997) Contributions to protein entropy and heat capacity from bond vector motions measured by NMR spin 
relaxation. J Mol Biol 272: 790-804.

Zalc, B. and Colman, DR. (2000) Orgins of vertebrate success. Science 288, 271-272.

Zhang, X, Sui, XG,Yang, DW (2006) Probing methyl dynamics from C-13 autocorrelated and cross-correlated relaxation. J Am Chem Soc 128: 5073-5081. 


\section{Appendix I}

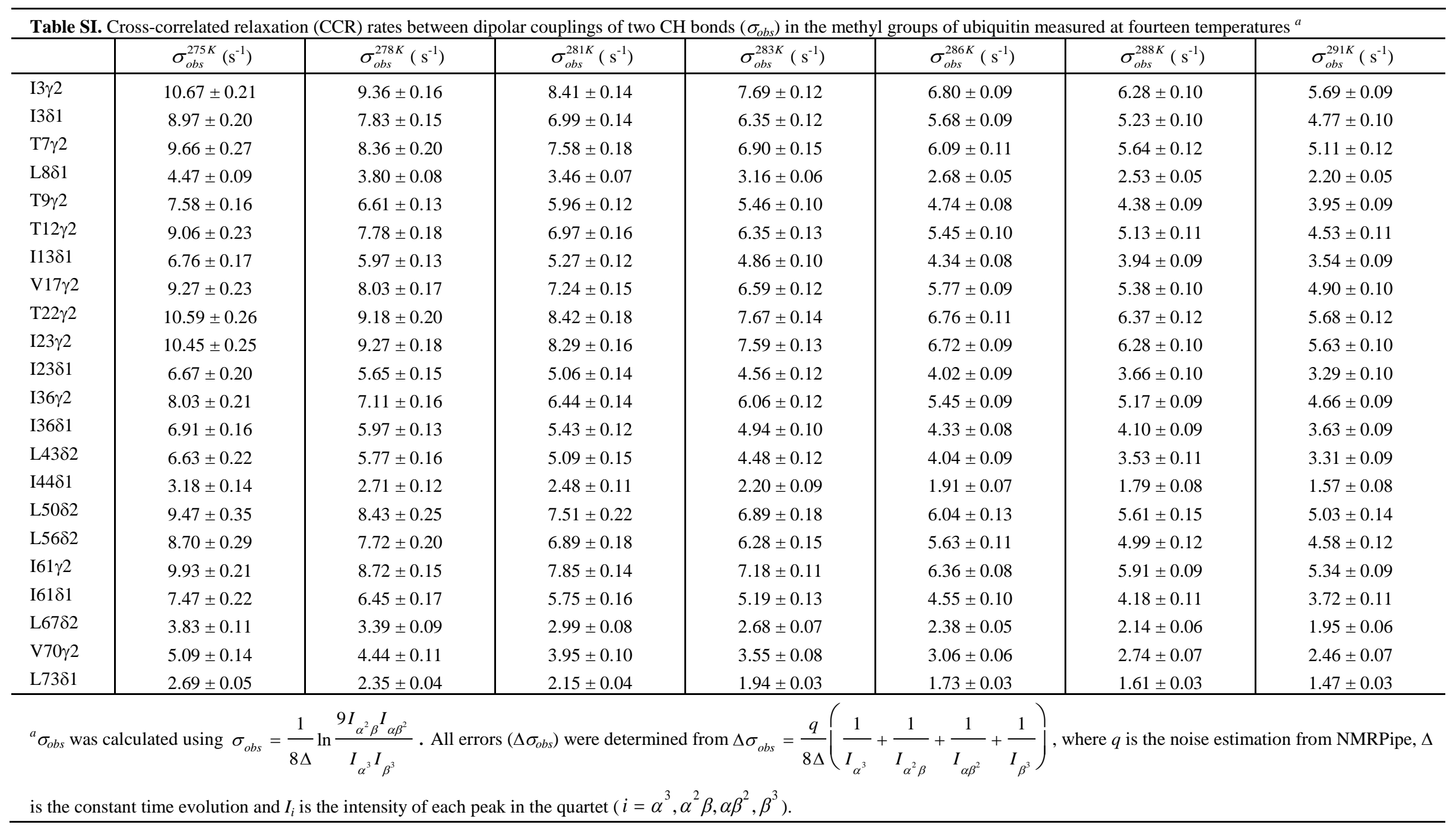




\begin{tabular}{|c|c|c|c|c|c|c|c|}
\hline & $\sigma_{o b s}^{293 K}\left(\mathrm{~s}^{-1}\right)$ & $\sigma_{o b s}^{296 K}\left(\mathrm{~s}^{-1}\right)$ & $\sigma_{o b s}^{298 K}\left(\mathrm{~s}^{-1}\right)$ & $\sigma_{o b s}^{301 K}\left(\mathrm{~s}^{-1}\right)$ & $\sigma_{o b s}^{303 K}\left(\mathrm{~s}^{-1}\right)$ & $\sigma_{o b s}^{305 K}\left(\mathrm{~s}^{-1}\right)$ & $\sigma_{o b s}^{308 K}\left(\mathrm{~s}^{-1}\right)$ \\
\hline $\mathrm{I} 3 \gamma 2$ & $5.34 \pm 0.07$ & $4.84 \pm 0.06$ & $4.52 \pm 0.07$ & $4.15 \pm 0.05$ & $3.94 \pm 0.06$ & $3.69 \pm 0.05$ & $3.42 \pm 0.05$ \\
\hline $\mathrm{I} 3 \delta 1$ & $4.46 \pm 0.08$ & $4.02 \pm 0.07$ & $3.77 \pm 0.07$ & $3.52 \pm 0.05$ & $3.24 \pm 0.06$ & $3.09 \pm 0.05$ & $2.83 \pm 0.05$ \\
\hline $\mathrm{T} 7 \gamma 2$ & $4.80 \pm 0.09$ & $4.43 \pm 0.08$ & $4.16 \pm 0.08$ & $3.90 \pm 0.06$ & $3.74 \pm 0.07$ & $3.52 \pm 0.06$ & $3.23 \pm 0.06$ \\
\hline L881 & $2.04 \pm 0.04$ & $1.84 \pm 0.04$ & $1.70 \pm 0.04$ & $1.55 \pm 0.03$ & $1.49 \pm 0.04$ & $1.44 \pm 0.03$ & $1.32 \pm 0.03$ \\
\hline Т9ү2 & $3.67 \pm 0.07$ & $3.32 \pm 0.06$ & $3.11 \pm 0.07$ & $2.79 \pm 0.05$ & $2.72 \pm 0.06$ & $2.54 \pm 0.05$ & $2.36 \pm 0.05$ \\
\hline $\mathrm{T} 12 \gamma 2$ & $4.20 \pm 0.09$ & $3.85 \pm 0.07$ & $3.60 \pm 0.08$ & $3.20 \pm 0.06$ & $3.09 \pm 0.07$ & $2.89 \pm 0.06$ & $2.63 \pm 0.06$ \\
\hline $\mathrm{I} 13 \delta 1$ & $3.29 \pm 0.07$ & $2.97 \pm 0.06$ & $2.76 \pm 0.07$ & $2.54 \pm 0.05$ & $2.35 \pm 0.06$ & $2.17 \pm 0.05$ & $2.04 \pm 0.05$ \\
\hline V17 $\gamma 2$ & $4.55 \pm 0.07$ & $4.09 \pm 0.06$ & $3.79 \pm 0.06$ & $3.46 \pm 0.04$ & $3.26 \pm 0.06$ & $3.13 \pm 0.04$ & $2.90 \pm 0.04$ \\
\hline $\mathrm{T} 22 \gamma 2$ & $5.26 \pm 0.09$ & $4.74 \pm 0.08$ & $4.48 \pm 0.08$ & $4.06 \pm 0.06$ & $3.82 \pm 0.07$ & $3.74 \pm 0.06$ & $3.35 \pm 0.06$ \\
\hline 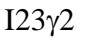 & $5.27 \pm 0.08$ & $4.79 \pm 0.06$ & $4.42 \pm 0.07$ & $4.13 \pm 0.05$ & $3.89 \pm 0.06$ & $3.71 \pm 0.05$ & $3.45 \pm 0.05$ \\
\hline I2381 & $3.03 \pm 0.08$ & $2.73 \pm 0.06$ & $2.54 \pm 0.07$ & $2.28 \pm 0.05$ & $2.11 \pm 0.06$ & $1.97 \pm 0.05$ & $1.79 \pm 0.05$ \\
\hline I36ү2 & $4.42 \pm 0.07$ & $4.02 \pm 0.06$ & $3.94 \pm 0.06$ & $3.54 \pm 0.04$ & $3.36 \pm 0.05$ & $3.19 \pm 0.04$ & $2.91 \pm 0.04$ \\
\hline I3681 & $3.41 \pm 0.07$ & $3.04 \pm 0.06$ & $2.82 \pm 0.06$ & $2.58 \pm 0.05$ & $2.44 \pm 0.06$ & $2.29 \pm 0.05$ & $2.12 \pm 0.05$ \\
\hline L4382 & $3.06 \pm 0.07$ & $2.76 \pm 0.06$ & $2.60 \pm 0.07$ & $2.37 \pm 0.05$ & $2.19 \pm 0.06$ & $2.01 \pm 0.05$ & $1.83 \pm 0.05$ \\
\hline $\mathrm{I} 44 \delta 1$ & $1.45 \pm 0.07$ & $1.24 \pm 0.06$ & $1.19 \pm 0.06$ & $1.03 \pm 0.04$ & $1.01 \pm 0.06$ & $0.92 \pm 0.05$ & $0.77 \pm 0.05$ \\
\hline L50 22 & $4.64 \pm 0.11$ & $4.16 \pm 0.09$ & $3.85 \pm 0.10$ & $3.43 \pm 0.07$ & $3.16 \pm 0.09$ & $3.02 \pm 0.07$ & $2.84 \pm 0.07$ \\
\hline L56 & $4.19 \pm 0.09$ & $3.82 \pm 0.08$ & $3.50 \pm 0.08$ & $3.17 \pm 0.06$ & $2.89 \pm 0.07$ & $2.73 \pm 0.06$ & $2.54 \pm 0.06$ \\
\hline I61 62 & $5.01 \pm 0.07$ & $4.57 \pm 0.06$ & $4.24 \pm 0.06$ & $3.91 \pm 0.04$ & $3.69 \pm 0.06$ & $3.49 \pm 0.04$ & $3.22 \pm 0.05$ \\
\hline I61 61 & $3.44 \pm 0.09$ & $3.03 \pm 0.07$ & $2.82 \pm 0.08$ & $2.56 \pm 0.05$ & $2.27 \pm 0.07$ & $2.13 \pm 0.06$ & $1.96 \pm 0.06$ \\
\hline L67 $\delta 2$ & $1.81 \pm 0.05$ & $1.64 \pm 0.04$ & $1.53 \pm 0.04$ & $1.37 \pm 0.03$ & $1.30 \pm 0.04$ & $1.18 \pm 0.03$ & $1.12 \pm 0.03$ \\
\hline V70 2 & $2.27 \pm 0.05$ & $2.07 \pm 0.05$ & $1.95 \pm 0.05$ & $1.80 \pm 0.04$ & $1.70 \pm 0.04$ & $1.64 \pm 0.04$ & $1.53 \pm 0.04$ \\
\hline L73 $\delta 1$ & $1.36 \pm 0.03$ & $1.25 \pm 0.02$ & $1.21 \pm 0.03$ & $1.10 \pm 0.02$ & $1.04 \pm 0.02$ & $1.00 \pm 0.02$ & $0.94 \pm 0.02$ \\
\hline & & $\frac{9 I_{\alpha^{2} \beta} I \beta^{2}}{I_{\alpha^{3}} I_{\beta^{3}}}$ & 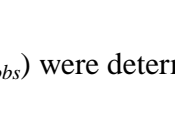 & $\sigma_{o b s}=\frac{q}{8 \Delta}$ & $+\frac{1}{I_{\alpha \beta^{2}}}+\frac{1}{I_{\beta^{3}}}$ & \multicolumn{2}{|c|}{, where $q$ is the noise estimation from NMRPipe, $\Delta$} \\
\hline
\end{tabular}


Table SII. Methyl group order parameters ( $S_{a x i s}^{2}$ ) calculated at fourteen temperatures ${ }^{a}$

\begin{tabular}{|c|c|c|c|c|c|c|c|c|c|c|c|c|c|c|}
\hline & \multicolumn{2}{|c|}{$275 \mathrm{~K}$} & \multicolumn{2}{|c|}{$278 \mathrm{~K}$} & \multicolumn{2}{|c|}{$281 \mathrm{~K}$} & \multicolumn{2}{|c|}{$283 \mathrm{~K}$} & \multicolumn{2}{|c|}{$286 \mathrm{~K}$} & \multicolumn{2}{|c|}{$288 \mathrm{~K}$} & \multicolumn{2}{|c|}{$291 \mathrm{~K}$} \\
\hline & $\tau_{c}(\mathrm{~ns})$ & 11.45 & $\tau_{c}(\mathrm{~ns})$ & 10.23 & $\tau_{c}(\mathrm{~ns})$ & 9.17 & $\tau_{c}(\mathrm{~ns})$ & 8.55 & $\tau_{c}(\mathrm{~ns})$ & 7.72 & $\tau_{c}(\mathrm{~ns})$ & 7.22 & $\tau_{c}(\mathrm{~ns})$ & 6.55 \\
\hline & $\sigma_{\text {riqid }}\left(\mathrm{s}^{-1}\right)$ & 10.95 & $\sigma_{\text {riqid }}\left(\mathrm{s}^{-1}\right)$ & 9.80 & $\sigma_{\text {riqid }}\left(\mathrm{s}^{-1}\right)$ & 8.79 & $\sigma_{\text {riqid }}\left(\mathrm{s}^{-1}\right)$ & 8.21 & $\sigma_{\text {rigid }}\left(\mathrm{s}^{-1}\right)$ & 7.42 & $\sigma_{\text {riqid }}\left(\mathrm{s}^{-1}\right)$ & 6.95 & $\sigma_{\text {rigid }}\left(\mathrm{s}^{-1}\right)$ & 6.32 \\
\hline & \multicolumn{2}{|c|}{$S_{\text {axis }}^{2}$} & \multicolumn{2}{|c|}{$S_{a x i s}^{2}$} & \multicolumn{2}{|c|}{$S_{a x i s}^{2}$} & \multicolumn{2}{|c|}{$S_{a x i s}^{2}$} & \multicolumn{2}{|c|}{$S_{a x i s}^{2}$} & \multicolumn{2}{|c|}{$S_{\text {axis }}^{2}$} & \multicolumn{2}{|c|}{$S_{a x i s}^{2}$} \\
\hline $\mathrm{I} 3 \gamma 2$ & \multirow{2}{*}{\multicolumn{2}{|c|}{$0.97 \pm 0.02$}} & \multicolumn{2}{|c|}{$0.96 \pm 0.02$} & \multicolumn{2}{|c|}{$0.96 \pm 0.02$} & \multicolumn{2}{|c|}{$0.94 \pm 0.01$} & \multicolumn{2}{|c|}{$0.92 \pm 0.01$} & \multicolumn{2}{|c|}{$0.90 \pm 0.01$} & \multicolumn{2}{|c|}{$0.90 \pm 0.01$} \\
\hline $\mathrm{I} 3 \delta 1$ & \multirow{2}{*}{\multicolumn{2}{|c|}{$\begin{array}{l}0.82 \pm 0.02 \\
0.88 \pm 0.02\end{array}$}} & \multicolumn{2}{|c|}{$0.80 \pm 0.02$} & \multicolumn{2}{|c|}{$0.80 \pm 0.02$} & \multicolumn{2}{|c|}{$0.77 \pm 0.01$} & \multicolumn{2}{|c|}{$0.77 \pm 0.01$} & \multicolumn{2}{|c|}{$0.75 \pm 0.01$} & \multicolumn{2}{|c|}{$0.75 \pm 0.02$} \\
\hline $\mathrm{T} 7 \gamma 2$ & & & 0.85 & 0.02 & 0.86 & .02 & 0.84 & .02 & 0.82 & & 0.81 & .02 & 0.81 & .02 \\
\hline L881 & \multicolumn{2}{|c|}{$0.41 \pm 0.01$} & 0.39 & 0.01 & 0.39 & .01 & 0.38 & .01 & 0.36 & & 0.36 & .01 & 0.35 & .01 \\
\hline Т9ү2 & 0.69 & 0.01 & 0.68 & 0.01 & 0.68 & .01 & 0.67 & .01 & 0.64 & & 0.63 & .01 & 0.63 & .01 \\
\hline $\mathrm{T} 12 \gamma 2$ & 0.83 & 0.02 & 0.79 & 0.02 & 0.79 & .02 & 0.77 & .02 & 0.73 & & 0.74 & .02 & 0.72 & .02 \\
\hline $\mathrm{I} 13 \delta 1$ & 0.62 & 0.02 & 0.61 & 0.01 & 0.60 & .01 & 0.59 & .01 & 0.58 & & 0.57 & .01 & 0.56 & .01 \\
\hline $\mathrm{V} 17 \gamma 2$ & 0.84 & 0.02 & 0.82 & 0.02 & $0.82=$ & .02 & 0.80 & .01 & $0.78=$ & & 0.77 & .01 & 0.78 & .02 \\
\hline $\mathrm{T} 22 \gamma 2$ & 0.97 & 0.02 & 0.94 & 0.02 & 0.96 & .02 & 0.93 & .02 & 0.91 & & 0.92 & .02 & 0.90 & .02 \\
\hline $\mathrm{I} 23 \gamma 2$ & 0.95 & 0.02 & 0.94 & 0.02 & 0.94 & .02 & 0.92 & .02 & 0.90 & & 0.90 & .01 & 0.89 & .02 \\
\hline $\mathrm{I} 23 \delta 1$ & 0.61 & 0.02 & 0.58 & 0.02 & 0.58 & .02 & 0.56 & .01 & 0.54 & & 0.53 & .01 & 0.52 & .02 \\
\hline 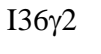 & 0.73 & 0.02 & 0.73 & 0.02 & 0.73 & .02 & 0.74 & .01 & 0.73 & & 0.74 & .01 & 0.74 & .01 \\
\hline I3681 & 0.63 & 0.02 & 0.61 & 0.01 & $0.62=$ & .01 & 0.60 & .01 & 0.58 & & 0.59 & .01 & 0.57 & .01 \\
\hline L4382 & 0.60 & 0.02 & 0.59 & 0.02 & 0.58 & .02 & 0.55 & .01 & 0.54 & & 0.51 & .02 & 0.52 & .02 \\
\hline $\mathrm{I} 44 \delta 1$ & 0.29 & 0.01 & 0.28 & 0.01 & 0.28 & .01 & 0.27 & .01 & 0.26 & & 0.26 & .01 & 0.25 & .01 \\
\hline L5082 & 0.87 & 0.03 & 0.86 & 0.03 & 0.85 & .03 & 0.84 & .02 & 0.81 & & 0.81 & .02 & 0.80 & .02 \\
\hline L5682 & 0.79 & 0.03 & 0.79 & 0.02 & 0.78 & .02 & 0.77 & .02 & 0.76 & & 0.72 & .02 & 0.73 & .02 \\
\hline I61 $\gamma 2$ & 0.91 & 0.02 & 0.89 & 0.02 & 0.89 & .02 & 0.87 & .01 & 0.86 & & 0.85 & .01 & 0.85 & .01 \\
\hline I61 $\delta 1$ & 0.68 & 0.02 & 0.66 & 0.02 & 0.65 & .02 & 0.63 & .02 & 0.61 & & 0.60 & .02 & 0.59 & .02 \\
\hline L67 22 & 0.35 & 0.01 & 0.35 & 0.01 & 0.34 & .01 & 0.33 & .01 & 0.32 & & 0.31 & .01 & 0.31 & .01 \\
\hline V70 2 & 0.46 & 0.01 & 0.45 & 0.01 & 0.45 & .01 & 0.43 & .01 & 0.41 & & 0.39 & .01 & 0.39 & .01 \\
\hline L7381 & 0.25 & 0.01 & 0.24 & 0.00 & $0.24=$ & .00 & 0.24 & .00 & $0.23=$ & .00 & 0.23 & .00 & 0.23 & .01 \\
\hline
\end{tabular}

${ }^{a}$ Given at each temperature are the rotational correlation time $\left(\tau_{c}\right)$ and the CCR rate between dipolar couplings of two CH bonds in the absence of local motion $\left(\sigma_{\text {rigid }}\right)$. $\sigma_{\text {rigid }}$ is calculated using $\sigma_{\text {rigid }}=\frac{1}{45} \tau_{c}\left(\frac{\mu_{0} h \gamma_{H} \gamma_{C}}{8 \pi^{2} r_{C H}^{3}}\right)^{2}\left(2+\frac{3}{2\left(1+\left(\omega_{c} \tau_{c}\right)^{2}\right)}\right)$, where $\mu_{\mathrm{o}}$ is the permeability of a vacuum, $h$ is the Planck constant, $\gamma_{H}$ and $\gamma_{C}$ are the gyromagnetic ratios of ${ }^{1} \mathrm{H}$ and ${ }^{13} \mathrm{C}$, respectively, $r_{C H}$ is the

$\mathrm{CH}$ bond length, $\omega_{C}$ is the Larmor frequency of ${ }^{13} \mathrm{C}$ and $\tau_{c}$ is the rotational correlation time. $S_{\text {axis }}^{2}$ is derived from $S_{\text {axis }}^{2}=\frac{\sigma_{\text {obs }}}{\sigma_{\text {rigid }}}$. The error $\left(\Delta S_{\text {axis }}^{2}\right)$ is determined using $\Delta S_{\text {axis }}^{2}=\frac{\Delta \sigma_{\text {obs }}}{\sigma_{\text {rigid }}}$. 


\begin{tabular}{|c|c|c|c|c|c|c|c|c|c|c|c|c|c|c|}
\hline & \multicolumn{2}{|c|}{$293 \mathrm{~K}$} & \multicolumn{2}{|c|}{$296 \mathrm{~K}$} & \multicolumn{2}{|c|}{$298 \mathrm{~K}$} & \multicolumn{2}{|c|}{$301 \mathrm{~K}$} & \multicolumn{2}{|c|}{$303 \mathrm{~K}$} & \multicolumn{2}{|c|}{$305 \mathrm{~K}$} & \multicolumn{2}{|c|}{$308 \mathrm{~K}$} \\
\hline & $\tau_{c}(\mathrm{~ns})$ & 6.15 & $\tau_{c}(\mathrm{~ns})$ & 5.61 & $\tau_{c}(\mathrm{~ns})$ & 5.28 & $\tau_{c}(\mathrm{~ns})$ & 4.84 & $\tau_{c}(\mathrm{~ns})$ & 4.57 & $\tau_{c}(\mathrm{~ns})$ & 4.32 & $\tau_{c}(\mathrm{~ns})$ & 3.98 \\
\hline & $\sigma_{\text {rigid }}\left(\mathrm{s}^{-1}\right)$ & 5.95 & $\sigma_{\text {rigid }}\left(\mathrm{s}^{-1}\right)$ & 5.44 & $\sigma_{\text {rigid }}\left(\mathrm{s}^{-1}\right)$ & 5.13 & $\sigma_{\text {rigid }}\left(\mathrm{s}^{-1}\right)$ & 4.72 & $\sigma_{\text {riqid }}\left(\mathrm{s}^{-1}\right)$ & 4.47 & $\sigma_{\text {riqid }}\left(\mathrm{s}^{-1}\right)$ & 4.24 & $\sigma_{\text {riqid }}\left(\mathrm{s}^{-1}\right)$ & 3.93 \\
\hline & \multicolumn{2}{|c|}{$S_{a x i s}^{2}$} & \multicolumn{2}{|c|}{$S_{a x i s}^{2}$} & \multicolumn{2}{|c|}{$S_{a x i s}^{2}$} & \multicolumn{2}{|c|}{$S_{a x i s}^{2}$} & \multicolumn{2}{|c|}{$S_{a x i s}^{2}$} & \multicolumn{2}{|c|}{$S_{a x i s}^{2}$} & \multicolumn{2}{|c|}{$S_{a x i s}^{2}$} \\
\hline $\mathrm{I} 3 \gamma 2$ & \multirow{2}{*}{\multicolumn{2}{|c|}{$0.90 \pm 0.01$}} & \multicolumn{2}{|c|}{$0.89 \pm 0.01$} & \multicolumn{2}{|c|}{$0.88 \pm 0.01$} & \multicolumn{2}{|c|}{$0.88 \pm 0.01$} & \multicolumn{2}{|c|}{$0.88 \pm 0.01$} & \multicolumn{2}{|c|}{$0.87 \pm 0.01$} & \multicolumn{2}{|c|}{$0.87 \pm 0.01$} \\
\hline $\mathrm{I} 3 \delta 1$ & & & \multicolumn{2}{|c|}{$0.74 \pm 0.01$} & \multicolumn{2}{|c|}{$0.73 \pm 0.01$} & \multicolumn{2}{|c|}{$0.74 \pm 0.01$} & \multicolumn{2}{|c|}{$0.72 \pm 0.01$} & \multicolumn{2}{|c|}{$0.73 \pm 0.01$} & \multicolumn{2}{|c|}{$0.72 \pm 0.01$} \\
\hline $\mathrm{T} 7 \gamma 2$ & \multirow{2}{*}{\multicolumn{2}{|c|}{$\begin{array}{l}0.81 \pm 0.02 \\
034+0.01\end{array}$}} & 0.81 & .01 & 0.81 & .02 & 0.82 & & 0.84 & .02 & 0.83 & .01 & 0.82 & 0.01 \\
\hline L8 81 & & & 0.34 & .01 & 0.33 & .01 & 0.33 & & 0.33 & .01 & 0.34 & .01 & 0.34 & 0.01 \\
\hline Т9 2 & 0.62 & 0.01 & 0.61 & .01 & 0.61 & .01 & 0.59 & & 0.61 & .01 & 0.60 & .01 & 0.60 & 0.01 \\
\hline $\mathrm{T} 12 \gamma 2$ & 0.71 & 0.01 & 0.71 & .01 & 0.70 & .02 & 0.68 & & 0.69 & .02 & 0.68 & .01 & 0.67 & 0.02 \\
\hline $\mathrm{I} 13 \delta 1$ & 0.55 & 0.01 & 0.55 & .01 & 0.54 & .01 & 0.54 & & 0.53 & .01 & 0.51 & .01 & 0.52 & 0.01 \\
\hline $\mathrm{V} 17 \gamma 2$ & 0.76 & 0.01 & 0.75 & .01 & 0.74 & .01 & 0.73 & & 0.73 & .01 & 0.74 & .01 & 0.74 & 0.01 \\
\hline $\mathrm{T} 22 \gamma 2$ & 0.88 & 0.02 & 0.87 & .01 & 0.87 & .02 & 0.86 & & 0.85 & .02 & 0.88 & .01 & 0.85 & 0.02 \\
\hline 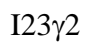 & 0.88 & 0.01 & 0.88 & .01 & 0.86 & .01 & 0.88 & & 0.87 & .01 & 0.88 & .01 & 0.88 & 0.01 \\
\hline $\mathrm{I} 23 \delta 1$ & 0.51 & 0.01 & 0.50 & .01 & 0.49 & .01 & 0.48 & & 0.47 & .01 & 0.46 & .01 & 0.46 & 0.01 \\
\hline 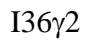 & 0.74 & 0.01 & 0.74 & .01 & 0.77 & .01 & 0.75 & & 0.75 & .01 & 0.75 & .01 & 0.74 & 0.01 \\
\hline I $36 \delta 1$ & 0.57 & 0.01 & 0.56 & .01 & 0.55 & .01 & 0.55 & & 0.55 & .01 & 0.54 & .01 & 0.54 & 0.01 \\
\hline $\mathrm{L} 43 \delta 2$ & 0.52 & 0.01 & 0.51 & .01 & 0.51 & .01 & 0.50 & & 0.49 & .01 & 0.47 & .01 & 0.47 & 0.01 \\
\hline $\mathrm{I} 44 \delta 1$ & 0.24 & 0.01 & 0.23 & .01 & 0.23 & .01 & 0.22 & & 0.23 & .01 & 0.22 & .01 & 0.20 & 0.01 \\
\hline L5082 & 0.78 & 0.02 & 0.76 & .02 & 0.75 & .02 & 0.73 & & 0.71 & .02 & 0.71 & .02 & 0.72 & 0.02 \\
\hline L56 $\delta 2$ & 0.70 & 0.02 & 0.70 & .01 & 0.68 & .02 & 0.67 & & 0.65 & .02 & 0.64 & .01 & 0.65 & 0.02 \\
\hline $\mathrm{I} 61 \gamma 2$ & 0.84 & 0.01 & 0.84 & .01 & 0.83 & .01 & 0.83 & & 0.82 & .01 & 0.82 & .01 & 0.82 & 0.01 \\
\hline I61ס1 & 0.58 & 0.01 & 0.56 & .01 & 0.55 & .02 & 0.54 & & 0.51 & .02 & 0.50 & .01 & 0.50 & 0.01 \\
\hline L67 62 & 0.30 & 0.01 & 0.30 & .01 & 0.30 & .01 & 0.29 & & 0.29 & .01 & 0.28 & .01 & 0.29 & 0.01 \\
\hline V70 2 & 0.38 & 0.01 & 0.38 & .01 & 0.38 & .01 & 0.38 & & 0.38 & .01 & 0.39 & .01 & 0.39 & 0.01 \\
\hline L73 1 & 0.23 & 0.01 & 0.23 & .00 & 0.24 & .01 & 0.23 & & 0.23 & .01 & 0.24 & .00 & 0.24 & 0.01 \\
\hline
\end{tabular}

${ }^{a}$ Given at each temperature are the rotational correlation time $\left(\tau_{c}\right)$ and the CCR rate between dipolar couplings of two CH bonds in the absence of local motion $\left(\sigma_{\text {rigid }}\right)$. $\sigma_{\text {rigid }}$ is calculated using $\sigma_{\text {rigid }}=\frac{1}{45} \tau_{c}\left(\frac{\mu_{0} h \gamma_{H} \gamma_{C}}{8 \pi^{2} r_{C H}^{3}}\right)^{2}\left(2+\frac{3}{2\left(1+\left(\omega_{c} \tau_{c}\right)^{2}\right)}\right)$, where $\mu_{o}$ is the permeability of a vacuum, $h$ is the Planck constant, $\gamma_{H}$ and $\gamma_{C}$ are the gyromagnetic ratios of ${ }^{1} \mathrm{H}$ and ${ }^{13} \mathrm{C}$, respectively, $r_{C H}$ is the $\mathrm{CH}$ bond length, $\omega_{C}$ is the Larmor frequency of ${ }^{13} \mathrm{C}$ and $\tau_{c}$ is the rotational correlation time. $S_{\text {axis }}^{2}$ is derived from $S_{\text {axis }}^{2}=\frac{\sigma_{\text {obs }}}{\sigma_{\text {rigid }}}$. The error $\left(\Delta S_{\text {axis }}^{2}\right)$ is determined using $\Delta S_{a x i s}^{2}=\frac{\Delta \sigma_{o b s}}{\sigma_{\text {rigid }}}$. 


\section{Appendix II $^{5}$}

Technical Resources - Media Formulations

Neurobasal ${ }^{\mathrm{TM}}$-A Medium (1X) liquid

Neurobasal ${ }^{\mathrm{TM}}$ Media are basal media formulated to meet the neuronal cells special requirements.

\begin{tabular}{|c|c|c|c|}
\hline Components & $\begin{array}{l}\text { Molecular } \\
\text { Weight }\end{array}$ & $\begin{array}{l}\text { Concentration } \\
\text { (mg/L) }\end{array}$ & $\mathbf{m M}$ \\
\hline \multicolumn{4}{|l|}{ Amino Acids } \\
\hline Glycine & 75 & 30 & 0.4 \\
\hline L-Alanine & 89 & 2 & 0.0225 \\
\hline L-Arginine hydrochloride & 211 & 84 & 0.398 \\
\hline L-Asparagine-H2O & 150 & 0.83 & 0.00553 \\
\hline L-Cysteine & 121 & 31.5 & 0.26 \\
\hline L-Histidine hydrochloride-H2O & 210 & 42 & 0.2 \\
\hline L-Isoleucine & 131 & 105 & 0.802 \\
\hline L-Leucine & 131 & 105 & 0.802 \\
\hline L-Lysine hydrochloride & 183 & 146 & 0.798 \\
\hline L-Methionine & 149 & 30 & 0.201 \\
\hline L-Phenylalanine & 165 & 66 & 0.4 \\
\hline L-Proline & 115 & 7.76 & 0.0675 \\
\hline L-Serine & 105 & 42 & 0.4 \\
\hline L-Threonine & 119 & 95 & 0.798 \\
\hline L-Tryptophan & 204 & 16 & 0.0784 \\
\hline L-Tyrosine & 181 & 72 & 0.398 \\
\hline L-Valine & 117 & 94 & 0.803 \\
\hline \multicolumn{4}{|l|}{ Vitamins } \\
\hline Choline chloride & 140 & 4 & 0.0286 \\
\hline D-Calcium pantothenate & 477 & 4 & 0.00839 \\
\hline Folic Acid & 441 & 4 & 0.00907 \\
\hline
\end{tabular}

${ }^{5}$ From Invitrogen ${ }^{\circledR}$ with modification used in this work from website: $\underline{\text { http://de- }}$ de.invitrogen.com/site/de/de/home/support/Product-Technical-Resources/media formulation.253.html 


\begin{tabular}{|c|c|c|c|}
\hline Niacinamide & 122 & 4 & 0.0328 \\
\hline Pyridoxine hydrochloride & 204 & 4 & 0.0196 \\
\hline Riboflavin & 376 & 0.4 & 0.00106 \\
\hline Thiamine hydrochloride & 337 & 4 & 0.0119 \\
\hline Vitamin B12 & 1355 & 0.0068 & 0.000005 \\
\hline i-Inositol & 180 & 7.2 & 0.04 \\
\hline \multicolumn{4}{|l|}{ Inorganic Salts } \\
\hline Calcium Chloride (CaCl2) (anhyd.) & 111 & 200 & 1.8 \\
\hline Ferric Nitrate (Fe(NO3)3"9H2O) & 404 & 0.1 & 0.000248 \\
\hline Magnesium Chloride (anhydrous) & 95 & 77.3 & 0.814 \\
\hline Potassium Chloride (KCl) & 75 & 400 & 5.33 \\
\hline Sodium Bicarbonate (NaHCO3) & 84 & 2200 & 26.19 \\
\hline Sodium Chloride ( $\mathrm{NaCl})$ & 58 & 4000 & 68.97 \\
\hline $\begin{array}{l}\text { Sodium Phosphate monobasic (NaH2PO4- } \\
\mathrm{H} 2 \mathrm{O} \text { ) }\end{array}$ & 138 & 125 & 0.906 \\
\hline Zinc sulfate (ZnSO4-7H2O) & 288 & 0.194 & 0.000674 \\
\hline \multicolumn{4}{|l|}{ Other Components } \\
\hline D-Glucose ${ }^{13} \mathrm{C}_{6}$ or ${ }^{12} \mathrm{C}_{6}$ & 180 & 4500 & 25 \\
\hline HEPES & 238 & 2600 & 10.92 \\
\hline Phenol Red & 110 & 8.1 & 0.0736 \\
\hline Sodium Pyruvate & 110 & 25 & 0.227 \\
\hline \multicolumn{4}{|l|}{ B27® supplement (2\%) } \\
\hline Glutamax ${ }^{\circledR}$ & & & 2 \\
\hline \multicolumn{4}{|l|}{ Penicillin (100 IU) } \\
\hline Streptomycin & & 100 & \\
\hline
\end{tabular}




\section{Curriculum Vitae}

Davood Bakhtiari

Personal information

Address

Phone

Fax

E-Mail

Date and place of Birth
Philipp-Reis-Str. 3, 37075 Göttingen

+49-551-2012250

+49-551-2012202

dbakhti@gwdg.de

September 17, 1981 in Kermanshah

Education

07/2009 - present

Ph.D. student in Max Planck Institute for Biophysical Chemistry, Göttingen, Germany

$04 / 2007-02 / 2009$

M.Sc. Medicinal Chemistry, Leibniz University Hannover, Germany/EMBL Heidelberg

$10 / 1999-03 / 2005$ Pharm.D., Pharmacy, Tehran University of medical sciences

Scientific skills

NMR spectroscopy, Cell biology, Organic synthesis

Computer skills

Python, Matlab, Mathematica, NMRPipe, CARA, Topspin,

XPIOR-NIH , CYANA,

Gromacs

Languages

Persian

Native

English

Fluent

German

Fluent

\section{Publications}

Sabo, TM., Bakhtiari D., Walter KFA., McFeeters RL., Giller, K., Becker S., Griesinger C., Lee, D. (2012) Thermal coefficient of methyl groups within ubiquitin. Protein Science 21: $562-570$.

Almasirad, A., Tajik, M., Bakhtiari, D., Shafiee, A., Abdollahi, M., Zamani, MJ., Khorasani, R., Esmaily H. (2005) Synthesis and analgesic activity of N-Arylhydrazone derivatives of mefenamic acid. Journal of pharmacy \& pharmaceutical sciences 8(3):419-25. 